\title{
Interactions in psychosis : linking environment, brain and genes
}

Citation for published version (APA):

Collip, D. (2011). Interactions in psychosis : linking environment, brain and genes. [Doctoral Thesis, Maastricht University]. Maastricht University. https://doi.org/10.26481/dis.20110914dc

Document status and date:

Published: 01/01/2011

DOI:

10.26481/dis.20110914dc

Document Version:

Publisher's PDF, also known as Version of record

\section{Please check the document version of this publication:}

- A submitted manuscript is the version of the article upon submission and before peer-review. There can be important differences between the submitted version and the official published version of record.

People interested in the research are advised to contact the author for the final version of the publication, or visit the DOI to the publisher's website.

- The final author version and the galley proof are versions of the publication after peer review.

- The final published version features the final layout of the paper including the volume, issue and page numbers.

Link to publication

\footnotetext{
General rights rights.

- You may freely distribute the URL identifying the publication in the public portal. please follow below link for the End User Agreement:

www.umlib.nl/taverne-license

Take down policy

If you believe that this document breaches copyright please contact us at:

repository@maastrichtuniversity.nl

providing details and we will investigate your claim.
}

Copyright and moral rights for the publications made accessible in the public portal are retained by the authors and/or other copyright owners and it is a condition of accessing publications that users recognise and abide by the legal requirements associated with these

- Users may download and print one copy of any publication from the public portal for the purpose of private study or research.

- You may not further distribute the material or use it for any profit-making activity or commercial gain

If the publication is distributed under the terms of Article $25 \mathrm{fa}$ of the Dutch Copyright Act, indicated by the "Taverne" license above, 


\section{Interactions in Psychosis \\ Linking environment, brain and genes}


(C) Dina Collip, Maastricht 2011

Cover Design | Sven Homes

Layout | Dina Collip \& Sven Homes

Print | Wöhrmann Print Service

ISBN | 978-90-8570-841-4 


\title{
Interactions in Psychosis \\ Linking environment, brain and genes
}

\author{
PROEFSCHRIFT
}

Ter verkrijging van de graad van doctor aan de Universiteit Maastricht, op gezag van de Rector Magnificus, Prof. Mr. G.P.M.F. Mols, volgens het besluit van het College van Decanen, in het openbaar te verdedigen op woensdag 14 september 2011 op 12:00

door

\section{Dina Collip}

Geboren op 27 augustus 1981 te Aachen 


\section{Promotores}

Prof. dr. I. Myin-Germeys

Prof. dr. J. van Os

\section{Beoordelingscommissie}

Prof. dr. M.W. deVries (voorzitter)

Prof. dr. R. Bentall (University of Liverpool)

Prof. Alison Yung (University of Melbourne)

Dr. Machteld Marcelis

Dr. Marieke Wichers

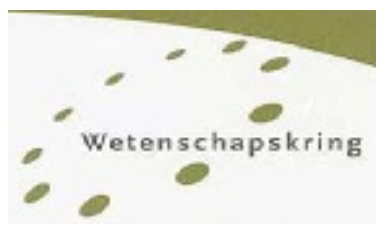

South Limburg Mental Health and Teaching Network PhD Series

The research presented in this thesis was conducted at the School for Mental Health and Neuroscience, Departement of Psychiatry and Neuropsychology of Maastricht University, Maastricht University Medical Centre and Mondriaan Zorggroep.

Publication of this thesis was financially supported by: Lundbeck B.V.

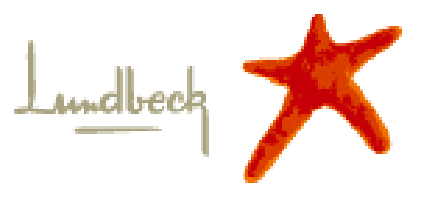


I am! Yet what I am who cares or knows?

My friends forsake me like a memory lost.

I am the self-consumer of my woes;

They rise and vanish, an oblivious host, Shadows of life, whose very soul is lost.

And yet I am - I live - though I am toss'd into the nothingness of scorn and noise, Into the living sea of waking dream, Where there is neither sense of life, nor joys,

But the huge shipwreck of my own esteem

And all that is dear. Even those I loved the best Are strange - nay, they are stranger than the rest.

I long for scenes where man has never trod For scenes where a woman never smiled or wept -

There to abide with my creator, God, And sleep as I in childhood sweetly slept, Full of high thoughts, unborn. So let me lie -

The grass below; above, the vaulted sky. 


\section{Paranimfen}

Margreet Oorschot

Johan Lataster 
Chapter 1 Introduction

p. 9

Chapter 2 Does the concept of "sensitization" provide a plausible mechanism for the putative link between the environment and schizophrenia?

Chapter 3 Dynamic association between interpersonal functioning \& positive symptom dimensions of psychosis over time: a longitudinal study of healthy adolescents.

Chapter 4 Social world interactions: how company connects to paranoia p. 51

Chapter 5 Daily cortisol, stress reactivity and psychotic experiences in individuals at above average genetic risk for psychosis.

Chapter 6 Hippocampal volume as marker of daily life emotional and cortisol stress sensitivity in psychosis.

Chapter 7 COMT Val158Met-stress interaction in psychosis: role of background psychosis risk.

Chapter 8 Epilogue

p. 131

Summary.

p. 143

Samenvatting

p. 148

Acknowledgements.

p. 153

Curriculum Vitae.

p. 159

List of publications

p. 160 



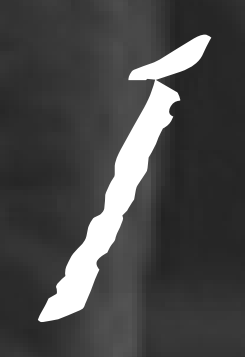

Introduction 


\section{Phenomenology of psychosis}

Psychotic disorders, including schizophrenia and other non-affective psychoses, are characterized by severe problems to recognize and understand reality. Among the core symptoms are positive symptoms, e.g. delusions and hallucinations, and negative symptoms, e.g. flat affect and anhedonia [see 1 for detailed description]. Besides positive and negative symptoms, alterations in functioning, including cognitive and social functioning, are a common characteristic of patients with schizophrenia or a first episode of psychotic illness [2, 3].

Schizophrenia, with a life time prevalence of $0.5-1 \%$ [4], is probably the most severe form of psychotic disorders, which affect about 2-3\% of the population [5]. It is a disorder of marked heterogeneity. The heterogeneity of the illness has traditionally been divided into two main forms [6-9], the positive syndrome, or non-deficit, type I schizophrenia and the negative syndrome, or deficit, type ll schizophrenia. The first is characterized by sudden onset, an episodic course, a good outcome, a good response to antipsychotic treatment, and high levels of positive symptoms, while the latter is characterized by a gradual onset, chronic course, poor outcome, resistance to drug treatment, high levels of negative symptoms and cognitive impairments [6-9].

In addition to this marked heterogeneity [10], psychotic experiences are thought to exist at levels below clinical expression, as subclinical psychotic experiences are commonly reported in the general population [11], particularly during adolescence, when expression of psychosis proneness peaks [12-14]. Therefore, the psychosis phenotype may be better understood as an extended psychosis phenotype. Subclinical psychotic experiences are mostly transient in nature [15-17], but in some individuals, psychotic experiences may be predictive of the development of psychotic disorder [16, 18]. According to the psychosis proneness - persistence - impairment model [19] the path from incidental psychotic experiences to clinical psychosis is mediated by the persistence of these experiences. Even though most subclinical psychotic experiences are transitory [20, 21], a familial and etiological continuity between subclinical psychotic experiences and psychotic disorders has been suggested. Etiological continuity refers to the notion that environmental factors implicated in clinical psychosis might be also important in eliciting subtle psychotic phenomena at preclinical stages of the psychosis continuum. Familial continuity indicates that the families of individuals with clinical psychotic disorder have higher rates of subclinical psychotic experiences and/or schizotypy, the milder and non-clinical form of psychosis at the level of personality structure [22]. Therefore, investigating psychotic symptoms in non-clinical populations with a higher than average risk of developing the disorder (psychometrically identified individuals with high schizotypy scores or first-degree relatives of patients with psychotic disorder) may help to increase the understanding of underlying mechanisms of psychotic symptoms and endophenotypes (e.g. increased stress sensitivity), without the problem of, for instance, potential confounding by antipsychotic treatment or effects of chronicity of psychotic illness. 
However, besides evidence supporting a psychosis continuum, the exact nature of this continuum still remains to be elucidated [23], including the identification of mechanisms and processes of transition over that continuum. While some studies suggest quantitative differences along the continuum, there might also be qualitative differences between those with subclinical symptomatology and those with established psychotic disorder, suggesting that studying mechanisms at different levels of psychosis risk may be valuable.

Clinical as well as subclinical psychotic experiences comprise broad concepts and have been divided into several symptom dimensions. Besides the possible classification into positive, negative, manic, depressive and disorganized symptoms and developmental cognitive alterations [24], there are several specific dimensions of positive psychotic symptoms such as hallucinations and delusions. Similarly, subclinical symptom dimensions have been identified, including persecutory ideation, bizarre experiences, perceptual abnormalities and magical thinking [13]. Bentall and Fernyhough have proposed that environmental influences impacting on psychotic experiences may display degrees of dimensional specificity [25], emphasizing the need to differentiate between symptom dimensions when studying underlying mechanisms. Therefore, one focus of this thesis was to differentially study positive symptom dimensions in association to environmental and genetic factors (Chapter 3, 4 \& 5).

\section{Affective pathway to psychosis}

In line with the idea of different subtypes of schizophrenia are the results of recent studies, which, in search of causal mechanisms and endophenotypes, investigated aberrations in sensitivity to stress as underlying vulnerability for psychotic disorders. In the face of everyday stress, patients with a psychotic disorder and their unaffected first-degree relatives have been found to display elevated emotional and behavioral responses [26]. It is suggested that this phenomenon, described as increased stress sensitivity, might be a sign of genetic and/or environmental liability to psychotic disorder [27]. Subsequent studies have shown that increased stress sensitivity clusters within families and might indeed be a vulnerability marker for psychosis $[28,29]$. Moreover, studies revealed that increased stress reactivity is independent from cognitive impairments and might even be a mutually exclusive mechanism or pathway to psychotic disorder [30-32]. While cognitive impairments seem to be predominantly associated with negative symptoms, increased stress reactivity has been found to be associated with positive psychotic symptoms [32]. Importantly, a genetic correlation between increased stress reactivity and the positive dimension of psychosis has been reported for clinical [29] as well as for subclinical psychotic experiences [28]. In addition, there is some evidence that part of the elevations in stress sensitivity can be explained by prior environmental exposures as life events [33] and childhood trauma [34]. Interestingly, these studies suggest that increased stress sensitivity characterizes a certain subgroup of patients that experience predominately positive psychotic symptoms. 
In conclusion, these studies provide evidence that increased sensitivity to stress is an endophenotype for psychosis. The underlying biological and genetic mechanisms of this increased stress sensitivity, however, have not satisfactorily been identified. Therefore, a main goal of the current thesis was to examine biological and genetic correlates of elevated stress sensitivity in psychosis (chapter 5, 6 \& 7).

\section{(Social) Environment and psychosis}

At present, there is overwhelming epidemiological evidence supporting the importance of environmental stress exposure in the developmental trajectory towards psychotic disorders [35] as well as for psychotic experiences in the general population [36]. Many of those factors, e.g. growing up in an urban environment [37, 38], experiencing discrimination [39] or childhood trauma [40] can be considered as indicators of social stress. Stress is thus an important factor in the etiology of psychosis, but many questions remain unanswered. None of the factors, for example, seems a necessary or sufficient cause to predict psychosis and it is unlikely that the diversity of environmental influences can be linked to as many different underlying mechanisms.

One possible approach to clarify the findings of epidemiological research may be to zoom into the microenvironment [41]. An excellent tool to study mechanisms at the microenvironment of everyday life is the Experience Sampling Method (ESM), as this enables the study of moment-to-moment changes of symptomatology in association with various contextual factors [42]. ESM is a structured self-assessment diary technique that is used to assess momentary context, thoughts, mood, and symptoms in the flow of daily life. Studies have demonstrated the feasibility, validity, and reliability of ESM in general and patient populations $[42,43]$. In chapter 4 , we present a study in which we used ESM to investigate whether social contextual variables are predictive of momentary increases in the intensity of paranoid thinking in a sample of participants ranging across the psychometric paranoia continuum.

Besides the external social environmental factors, there is also evidence for more internal trait-like problems, including alterations in social and interpersonal functioning. Clinical improvement in patients is often independent of improvements in social functioning, suggesting that alterations in social functioning may be a continuation of poor premorbid functioning [44]. This is in line with evidence of alterations in premorbid social development in childhood [45-47] and adolescence [48] in individuals who later develop non-affective psychotic disorder. Individuals at UHR who make the transition to an overt psychotic outcome also show alterations in social functioning compared to individuals who do not develop clinical psychotic states [49-52]. Furthermore, at the level of the general population, the persistence of subclinical psychotic experiences is similarly associated with poorer social functioning [16]. Together, these findings suggest that alterations in social functioning may impact on development of psychotic illness in those at risk. However, several issues remain. For instance, most studies only addressed one direction-social functioning predicting psychosis rather than the other way round-or used 
a cross-sectional design, which precludes observing how the variables are related over time. Moreover, most studies have looked at overall psychotic symptomatology or psychotic illness as outcome, but not at separate dimensions of symptoms. Most importantly, the majority of studies were conducted in UHR or patient populations. In individuals further along the extended psychosis continuum, it may be more difficult to draw conclusions, because the very presence of clinical psychotic experiences may color the interpretation of (neutral) social stimuli. In chapter $\mathbf{3}$ a study is presented, in which we extend results of the association between social functioning and psychotic symptoms in at-risk and patient populations to a general adolescent sample, considering differences between different symptom dimensions in the association with interpersonal functioning.

\section{Biological underpinnings}

The biological substrates underlying the phenomenon of increased stress sensitivity or the link between stress and psychotic experiences remain largely unknown, and vulnerability markers need to be identified. Results of experimental studies suggest that increased psychotic reactivity to stress may reflect increased dopamine reactivity $[53,54]$. In addition, several lines of evidence indicate that dysregulation of the hypothalamic-pituitary-adrenocortical (HPA) axis may play a role in the relationship between stress and psychotic experiences $[55,56]$. In individuals with a psychotic disorder, abnormalities have been found at various levels of the HPA axis, including functional and structural changes in the hypothalamus [57], the pituitary [58], and the hippocampus [59]. More specifically, patients with psychotic disorder display reduced hippocampal size [60-63]. Recent studies have shown that similar alterations in hippocampal volume (HV) are present in first-degree relatives of patients with schizophrenia [64, 65], suggesting that HV changes constitute part of the liability to psychosis. The hippocampus plays a pivotal role in regulating emotional responses to stressful stimuli and in the negative feedback mechanism on HPA axis activity [66-70], and is therefore an interesting candidate in search for biological underpinnings of increased stress sensitivity.

Similarly, diurnal cortisol levels and cortisol reactivity to stress may be disturbed in patients with psychotic disorder [71-79], although results are inconsistent. Moreover, patient status and use of medication introduce difficulties for the interpretation of the results: are cortisol alterations a state marker, a trait marker or a result of antipsychotic medication? Therefore, studies in individuals at increased psychometric risk for psychotic disorder were conducted. Schizotypal adolescents, for example, were found to have higher cortisol levels than controls [80], and heightened cortisol secretion in another sample of schizotypal adolescents was associated with schizotypal symptomatology at 2-year follow-up [81]. The potential predictive value of cortisol alterations is further supported by findings in a sample of young people at increased risk of psychotic disorder; Walker et al. (2010) recently reported that higher cortisol levels predicted higher risk of conversion to psychotic disorder in an at-risk sample studied 
longitudinally. However, cortisol reactivity to daily life stress in a sample at heightened genetic risk for psychosis has never been investigated.

Together these findings add support to the hypothesis that HPA axis abnormalities may underlie vulnerability to psychotic disorder and potentially also to heightened stress reactivity. Consequently, we examined daily life cortisol patterns in siblings of patients with a psychotic disorder compared to healthy controls. Besides examination of the diurnal slope and cortisol reactivity in response to naturally occurring stressors, we investigated the association between momentary psychotic experiences and momentary cortisol secretion. Results of this study are presented in chapter $\mathbf{5}$. Moreover, the study presented in chapter 6 investigated the association between hippocampal volume and emotional and cortisol reactivity to daily life stress in a sample of individuals with different degrees of psychosis liability.

\section{Gene-environment interactions}

Heterogeneity in behavioral and biological responses to environmental stressors can best be understood in the framework of the vulnerability-stress model [82]. According to the vulnerability-stress model, sensitivity to environmental stressors depends on the genetic liability of an individual. This approach has led to a series of studies investigating gene-environment interactions in the search for mechanisms of stress sensitivity and the development of positive psychotic symptoms [e.g. 83].

Moffitt et al. (2005) recommended a prospective collection of cumulative, repeated measures of proximal rather than distal environmental risk factors to optimize the environmental risk measurement in gene-environment research [84]. ESM has therefore been proposed as an excellent tool to study gene-environment interactions [42]. This is confirmed by a few studies of our group that used ESM to study gene-environment interactions in psychopathology [85-87].

In individuals at increased genetic risk for psychosis, augmented stress sensitivity in daily life has been associated with an augmented dopamine response to a physiological stressor [88]. Therefore, genetic variation in the Val158Met functional polymorphism of the catechol-O-methyltransferase (COMT) gene is an obvious candidate to study genetic moderation of environmental stress in psychosis. The COMT gene encodes an enzyme that is critical in the breakdown of dopamine, especially in the prefrontal cortex, and brain COMT enzyme activity in individuals with the Val/Val genotype is about $40 \%$ higher than in individuals with Met/Met genotype [89].

While the Val-allele carriers have been found to be more sensitive to the psychotogenic effects of cannabis [87], results of some COMT-stress interaction studies point towards exaggerated sensitivity to stress in Met-carriers $[90,91]$. The first ESM study in patients with psychotic disorder showed that 
patients with the Met/Met genotype reported the largest increase in psychotic symptoms and negative emotions in response to daily stressors, thus providing evidence for gene-environment interaction mechanisms in the formation of psychotic symptoms [86]. Interestingly, this effect was not found in the healthy control group and a second study in a general population sample found opposite results, with $\mathrm{Val} / \mathrm{Val}$ carriers displaying more feelings of paranoia in response to stress [92]. Thus, COMT Val158Met interactions with stress may be contingent on background genetic risk for psychotic disorder, revealing the need for replication studies. Consequently, in chapter 7 , such a replication study is presented including patients with a psychotic disorder as well as healthy controls allowing the examination of disease specific effects.

\section{Aims of this thesis}

The overall aim of this thesis was to further explore potential mechanisms underlying clinical and subclinical positive psychotic symptoms as well as the mechanisms underlying increased stress sensitivity, including contextual effects (social factors), biological markers and genetic underpinnings in the form of gene-environment interactions.

This led to the following research:

In chapter 2, literature is reviewed about environmental effects on psychosis. We propose the concept of sensitization as a possible link between environment and psychosis and discuss potential biological correlates of sensitization processes. As epidemiological studies suggest that social factors may be important risk factors for psychosis, we investigated the bi-directional, longitudinal association between interpersonal functioning and different positive psychotic symptom dimensions in a general population adolescent sample (chapter $\mathbf{3}$ ). In chapter $\mathbf{4}$, we zoomed in into the microenvironment of daily life in order to understand effects of social context on momentary paranoia at different levels of the paranoia phenotype.

A further aim was to investigate biological correlates of the association between stress and psychosis and of increased stress sensitivity. In chapter $\mathbf{5}$, cortisol patterns in the context of daily life were examined in siblings of patients with a psychotic disorder compared to healthy controls. This included, among others, examination of the diurnal slope, cortisol reactivity in response to naturally occurring stressors, and the association between momentary psychotic experiences and momentary cortisol. In chapter 6, we investigated the role of hippocampal volume in association with levels of emotional and cortisol reactivity to daily life stress and the hypothesis whether this association was different between groups with different degrees of risk for psychotic disorder.

Genetic variation might act synergistically with environmental stress in eliciting psychotic symptoms. In chapter 7, we aimed to replicate the association between the COMT gene and stress sensitivity and to clarify whether it might be moderated by psychosis liability. 


\section{References}

1. APA (1994): Diagnostic and Statistical Manual of Mental Disorders, Fourth Edition. 1994, Washington, DC: American Psychiatric Association.

2. Shim G, Kang DH, Sun Chung Y, Young Yoo S, Young Shin N, and Soo Kwon J, (2008): Social functioning deficits in young people at risk for schizophrenia. Informa UK Ltd UK. p. 678-685.

3. Addington J, Penn D, Woods SW, Addington D, and Perkins DO, (2008): Social functioning in individuals at clinical high risk for psychosis. Elsevier. p. 119-124.

4. McGrath J, Saha S, Welham J, El Saadi O, MacCauley C, and Chant D (2004): A systematic review of the incidence of schizophrenia: the distribution of rates and the influence of sex, urbanicity, migrant status and methodology. $B M C$ Med 2:13.

5. Perala J, Suvisaari J, Saarni SI, Kuoppasalmi K, Isometsa E, Pirkola S, et al. (2007): Lifetime prevalence of psychotic and bipolar I disorders in a general population. Arch Gen Psychiatry 64:19-28.

6. Andreasen NC (1985): Positive vs. negative schizophrenia: a critical evaluation. Schizophr Bull 11:380-9.

7. Crow TJ (1980): Molecular pathology of schizophrenia: more than one disease process? Br Med J 280:66-8.

8. Murray RM, O'Callaghan E, Castle DJ, and Lewis SW (1992): A neurodevelopmental approach to the classification of schizophrenia. Schizophr Bull 18:319-32.

9. Robins E and Guze SB (1970): Establishment of diagnostic validity in psychiatric illness: its application to schizophrenia. Am J Psychiatry 126:983-7.

10. Johns LC and van Os J (2001): The continuity of psychotic experiences in the general population. Clin Psychol Rev 21:1125-41.

van Os J, Linscott RJ, Myin-Germeys I, Delespaul P, and Krabbendam L (2009): A systematic review and meta-analysis of the psychosis continuum: evidence for a psychosis proneness-persistence-impairment model of psychotic disorder. Psychol Med 39:179-95.

12. Verdoux H, Van Os J, Maurice-Tison S, Gay B, Salamon R, and Bourgeois M, (1998): Is early adulthood a critical developmental stage for psychosis proneness? A survey of delusional ideation in normal subjects. Elsevier. p. 247254.

13. Yung AR, Nelson B, Baker K, Buckby JA, Baksheev G, and Cosgrave EM, (2009): Psychotic-like experiences in a community sample of adolescents: implications for the continuum model of psychosis and prediction of schizophrenia. p. 118-128.

14. Wigman JT, Vollebergh WA, Raaijmakers QA, ledema J, van Dorsselaer S, Ormel J, et al. (2009): The Structure of The Extended Psychosis Phenotype in Early Adolescence--A Cross-sample Replication. Schizophr Bull.

15. Dhossche D, Ferdinand R, Van der Ende J, Hofstra MB, and Verhulst F, (2002): Diagnostic outcome of self-reported hallucinations in a community sample of adolescents. Cambridge Univ Press. p. 619-627.

16. Dominguez MG, Wichers M, Lieb R, Wittchen HU, and van Os J, (2009): Evidence that onset of clinical psychosis is an outcome of progressively more persistent subclinical psychotic experiences: an 8-year cohort study. MPRC.

17. Wiles NJ, Zammit S, Bebbington P, Singleton N, Meltzer H, and Lewis G (2006): Self-reported psychotic symptoms in the general population: results from the longitudinal study of the British National Psychiatric Morbidity Survey. $\mathrm{Br}$ J Psychiatry 188:519-26.

18. Poulton R, Caspi A, Moffitt TE, Cannon M, Murray R, and Harrington H (2000): Children's self-reported psychotic symptoms and adult schizophreniform disorder: a 15-year longitudinal study. Arch Gen Psychiatry 57:1053-8.

19. Cougnard A, Marcelis M, Myin-Germeys I, De Graaf R, Vollebergh W, Krabbendam L, et al. (2007): Does normal developmental expression of psychosis combine with environmental risk to cause persistence of psychosis? A psychosis proneness-persistence model. Psychol Med:1-15. 
psychosis proneness-persistence model. Psychol Med 37:513-27.

21. Hanssen M, Bak M, Bijl R, Vollebergh W, and van Os J (2005): The incidence and outcome of subclinical psychotic experiences in the general population. British Journal of Clinical Psychology 44:181-91.

22. Johns LC and van Os J (2001): The continuity of psychotic experiences in the general population. Clin Psychol Rev 21:1125-41.

23. David AS (2010): Why we need more debate on whether psychotic symptoms lie on a continuum with normality. Psychol Med 40:1935-42.

24. Van Os J, Are psychiatric diagnoses of psychosis scientific and useful? The case of schizophrenia. Informa UK Ltd London, UK. p. 305-317.

25. Bentall RP and Fernyhough C, (2008): Social predictors of psychotic experiences: specificity and psychological mechanisms. MPRC.

26. Myin-Germeys I, Delespaul P, and van Os J (2005): Behavioural sensitization to daily life stress in psychosis. Psychological Medicine 35:733-41.

27. Myin-Germeys I and van Os J (2007): Stress-reactivity in psychosis: evidence for an affective pathway to psychosis. Clin Psychol Rev 27:409-24.

28. Lataster T, Wichers M, Jacobs N, Mengelers R, Derom C, Thiery E, et al. (2009): Does reactivity to stress cosegregate with subclinical psychosis? A general population twin study. Acta Psychiatr Scand 119:45-53.

29. Lataster T, Collip D, Lardinois M, van Os J, and Myin-Germeys I (2010): Evidence for a familial correlation between increased reactivity to stress and positive psychotic symptoms. Acta Psychiatr Scand 122:395-404.

30. Myin-Germeys I, Krabbendam L, Jolles J, Delespaul PA, and van Os J (2002): Are cognitive impairments associated with sensitivity to stress in schizophrenia? An experience sampling study. The American Journal of Psychiatry 159:443-9.

31. Morrens M, Krabbendam L, Bak M, Delespaul P, Mengelers R, Sabbe B, et al. (2007): The relationship between cognitive dysfunction and stress sensitivity in schizophrenia: a replication study. Soc Psychiatry Psychiatr Epidemiol 42:284-7.

32. Lataster T, Valmaggia, L., Lardinois, M., Mengelers, R., van Os, J. \& Myin-Germeys, I. (submitted): Stress-reactivity and cognitive impairments: separate mechanisms underlying different symptoms in psychosis.

33. Myin-Germeys I, Krabbendam L, Delespaul PA, and Van Os J (2003): Do life events have their effect on psychosis by influencing the emotional reactivity to daily life stress? Psychol Med 33:327-33.

34. Lardinois M, Lataster T, Mengelers R, Van Os J, and Myin-Germeys I (2011): Childhood trauma and increased stress sensitivity in psychosis. Acta Psychiatr Scand 123:28-35.

35. van Winkel R, Stefanis NC, and Myin-Germeys I (2008): Psychosocial stress and psychosis. A review of the neurobiological mechanisms and the evidence for gene-stress interaction. Schizophr Bull 34:1095-105.

36. Johns LC, Cannon M, Singleton N, Murray RM, Farrell M, Brugha T, et al. (2004): Prevalence and correlates of selfreported psychotic symptoms in the British population. Br J Psychiatry 185:298-305.

37. van Os J, Hanssen M, Bak M, Bijl RV, and Vollebergh W (2003): Do urbanicity and familial liability coparticipate in causing psychosis? Am J Psychiatry 160:477-82.

38. van Os J, Hanssen M, Bijl RV, and Vollebergh W (2001): Prevalence of psychotic disorder and community level of psychotic symptoms: an urban-rural comparison. Arch Gen Psychiatry 58:663-8.

39. Veling W, Selten JP, Veen N, Laan W, Blom JD, and Hoek HW (2006): Incidence of schizophrenia among ethnic minorities in the Netherlands: a four-year first-contact study. Schizophr Res 86:189-93.

40. Read J, van Os J, Morrison AP, and Ross CA (2005): Childhood trauma, psychosis and schizophrenia: a literature review with theoretical and clinical implications. Acta Psychiatrica Scandinavica 112:330-50.

41. McGrath JJ (2007): The surprisingly rich contours of schizophrenia epidemiology. Arch Gen Psychiatry 64:14-6. 
42. Myin-Germeys I, Oorschot M, Collip D, Lataster J, Delespaul P, and van Os J (2009): Experience sampling research in psychopathology: opening the black box of daily life. Psychol Med 39:1533-47.

43. Csikszentmihalyi M and Larson R (1987): Validity and reliability of the Experience-Sampling Method. Journal of Nervous and Mental Disease 175:526-36.

44. Shrivastava A, Johnston M, Shah N, and Bureau Y (2010): Redefining outcome measures in schizophrenia: integrating social and clinical parameters. Curr Opin Psychiatry 23:120-6.

45. Cannon M, Caspi A, Moffitt TE, Harrington H, Taylor A, Murray RM, et al. (2002): Evidence for early-childhood, pandevelopmental impairment specific to schizophreniform disorder: results from a longitudinal birth cohort. Arch Gen Psychiatry 59:449-56.

46. Jones P, Murray R, Rodgers B, and Marmot M, (1994): Child developmental risk factors for adult schizophrenia in the British 1946 birth cohort. Elsevier. p. 1398-1402.

47. Dworkin RH, Cornblatt BA, Friedmann R, Kaplansky LM, Lewis JA, Rinaldi A, et al. (1993): Childhood precursors of affective vs. social deficits in adolescents at risk for schizophrenia. Schizophr Bull 19:563-77.

48. Davidson M, Reichenberg A, Rabinowitz J, Weiser M, Kaplan Z, and Mark M (1999): Behavioral and intellectual markers for schizophrenia in apparently healthy male adolescents. Am J Psychiatry 156:1328-35.

49. Velthorst E, Nieman DH, Becker HE, van de Fliert R, Dingemans PM, Klaassen R, et al., (2009): Baseline differences in clinical symptomatology between ultra high risk subjects with and without a transition to psychosis. Elsevier. $\mathrm{p}$. 60-65.

50. Cornblatt BA, Auther AM, Niendam T, Smith CW, Zinberg J, Bearden CE, et al., (2007): Preliminary findings for two new measures of social and role functioning in the prodromal phase of schizophrenia. MPRC. p. 688.

51. Mason O, Startup M, Halpin S, Schall U, Conrad A, and Carr V (2004): Risk factors for transition to first episode psychosis among individuals with 'at-risk mental states'. Schizophr Res 71:227-37.

52. Yung AR, McGorry PD, McFarlane CA, Jackson HJ, Patton GC, and Rakkar A (1996): Monitoring and care of young people at incipient risk of psychosis. Schizophr Bull 22:283-303.

53. Myin-Germeys I, Marcelis M, Krabbendam L, Delespaul P, and van Os J (2005): Subtle fluctuations in psychotic phenomena as functional states of abnormal dopamine reactivity in individuals at risk. Biological Psychiatry 58:10510.

54. Soliman A, O'Driscoll GA, Pruessner J, Holahan AL, Boileau I, Gagnon D, et al. (2008): Stress-induced dopamine release in humans at risk of psychosis: a [11C]raclopride PET study. Neuropsychopharmacology 33:2033-41.

van Winkel R, Stefanis NC, and Myin-Germeys I (2008): Psychosocial stress and psychosis. A review of the neurobiological mechanisms and the evidence for gene-stress interaction. Schizophrenia Bulletin 34:1095-105.

56. Walker E, Mittal V, and Tessner K (2008): Stress and the hypothalamic pituitary adrenal axis in the developmental course of schizophrenia. Annual Review of Clinical Psychology 4:189-216.

57. Koolschijn PC, van Haren NE, Hulshoff Pol HE, and Kahn RS (2008): Hypothalamus volume in twin pairs discordant for schizophrenia. European Neuropsychopharmacology 18:312-5.

58. Pariante CM (2008): Pituitary volume in psychosis: the first review of the evidence. Journal of Psychopharmacology 22:76-81.

59. Wright IC, Rabe-Hesketh S, Woodruff PW, David AS, Murray RM, and Bullmore ET (2000): Meta-analysis of regional brain volumes in schizophrenia. American Journal of Psychiatry 157:16-25.

60. Geuze E, Vermetten E, and Bremner JD (2005): MR-based in vivo hippocampal volumetrics: 2. Findings in neuropsychiatric disorders. Mol Psychiatry 10:160-84.

61. Steen RG, Mull C, McClure R, Hamer RM, and Lieberman JA (2006): Brain volume in first-episode schizophrenia: systematic review and meta-analysis of magnetic resonance imaging studies. Br J Psychiatry 188:510-8.

62. Wright IC, Rabe-Hesketh S, Woodruff PW, David AS, Murray RM, and Bullmore ET (2000): Meta-analysis of regional brain volumes in schizophrenia. Am J Psychiatry 157:16-25. 
63. Vita A, De Peri L, Silenzi C, and Dieci M (2006): Brain morphology in first-episode schizophrenia: a meta-analysis of quantitative magnetic resonance imaging studies. Schizophr Res 82:75-88.

64. Lawrie SM, Mclntosh AM, Hall J, Owens DG, and Johnstone EC (2008): Brain structure and function changes during the development of schizophrenia: the evidence from studies of subjects at increased genetic risk. Schizophr Bull 34:330-40.

65. Boos H, Aleman A, Cahn W, Pol HH, and Kahn RS, (2007): Brain volumes in relatives of patients with schizophrenia: a meta-analysis. Am Med Assoc. p. 297.

66. Corcoran C, Walker E, Huot R, Mittal V, Tessner K, Kestler L, et al. (2003): The stress cascade and schizophrenia: etiology and onset. Schizophr Bull 29:671-92.

67. O'Brien JT, (1997): The'glucocorticoid cascade'hypothesis in man: prolonged stress may cause permanent brain damage. Maney. p. 199-201.

68. Sapolsky RM, (2000): Glucocorticoids and hippocampal atrophy in neuropsychiatric disorders. Am Med Assoc. p. 925.

69. Buchanan TW, Tranel D, and Kirschbaum C (2009): Hippocampal damage abolishes the cortisol response to psychosocial stress in humans. Horm Behav 56:44-50.

70. Jacobson L and Sapolsky R (1991): The role of the hippocampus in feedback regulation of the hypothalamicpituitary-adrenocortical axis. Endocr Rev 12:118-34.

71. Ryan MC, Sharifi N, Condren R, and Thakore JH (2004): Evidence of basal pituitary-adrenal overactivity in first episode, drug naive patients with schizophrenia. Psychoneuroendocrinology 29:1065-70.

72. Jansen LM, Gispen-de Wied CC, Gademan PJ, De Jonge RC, van der Linden JA, and Kahn RS (1998): Blunted cortisol response to a psychosocial stressor in schizophrenia. Schizophrenia Research 33:87-94.

73. Jansen LM, Gispen-de Wied CC, and Kahn RS (2000): Selective impairments in the stress response in schizophrenic patients. Psychopharmacology (Berlin) 149:319-25.

74. Marcelis M, Cavalier E, Gielen J, Delespaul P, and Van Os J (2004): Abnormal response to metabolic stress in schizophrenia: marker of vulnerability or acquired sensitization? Psychological Medicine 34:1103-11.

75. Breier A, Wolkowitz OM, Doran AR, Bellar S, and Pickar D (1988): Neurobiological effects of lumbar puncture stress in psychiatric patients and healthy volunteers. Psychiatry Research 25:187-94.

76. Albus M, Ackenheil M, Engel RR, and Muller F (1982): Situational reactivity of autonomic functions in schizophrenic patients. Psychiatry Research 6:361-70.

77. Brenner K, Liu A, Laplante DP, Lupien S, Pruessner JC, Ciampi A, et al. (2009): Cortisol response to a psychosocial stressor in schizophrenia: blunted, delayed, or normal? Psychoneuroendocrinology 34:859-68.

78. Walsh P, Spelman L, Sharifi N, and Thakore JH (2005): Male patients with paranoid schizophrenia have greater ACTH and cortisol secretion in response to metoclopramide-induced AVP release. Psychoneuroendocrinology 30:431-7.

79. Mondelli V, Dazzan P, Hepgul N, Di Forti M, Aas M, D’Albenzio A, et al. (2010): Abnormal cortisol levels during the day and cortisol awakening response in first-episode psychosis: the role of stress and of antipsychotic treatment. Schizophrenia Research 116:234-42.

80. Mittal VA, Dhruv S, Tessner KD, Walder DJ, and Walker EF (2007): The relations among putative biorisk markers in schizotypal adolescents: minor physical anomalies, movement abnormalities, and salivary cortisol. Biological Psychiatry 61:1179-86.

81. Walker EF, Walder DJ, and Reynolds F (2001): Developmental changes in cortisol secretion in normal and at-risk youth. Development and Psychopathology 13:721-32.

82. Nuechterlein KH and Dawson ME (1984): A heuristic vulnerability/stress model of schizophrenic episodes. Schizophr Bull 10:300-12.

83. Stefanis NC, Henquet C, Avramopoulos D, Smyrnis N, Evdokimidis I, Myin-Germeys I, et al. (2007): COMT Val158Met moderation of stress-induced psychosis. Psychol Med 37:1651-6. 
84. Moffitt TE, Caspi A, and Rutter M (2005): Strategy for investigating interactions between measured genes and measured environments. Arch Gen Psychiatry 62:473-81.

85. Wichers M, Kenis G, Jacobs N, Myin-Germeys I, Schruers K, Mengelers R, et al. (2008): The psychology of psychiatric genetics: evidence that positive emotions in females moderate genetic sensitivity to social stress associated with the BDNF Val-sup-6-sup-6Met polymorphism. J Abnorm Psychol 117:699-704.

86. van Winkel R, Henquet C, Rosa A, Papiol S, Fananas L, De Hert M, et al. (2008): Evidence that the COMT(Val158Met) polymorphism moderates sensitivity to stress in psychosis: an experience-sampling study. Am J Med Genet $B$ Neuropsychiatr Genet 147B:10-7.

87. Henquet C, Rosa A, Delespaul P, Papiol S, Fananas L, van Os J, et al. (2009): COMT ValMet moderation of cannabisinduced psychosis: a momentary assessment study of 'switching on' hallucinations in the flow of daily life. Acta Psychiatr Scand 119:156-60.

88. Myin-Germeys I, Marcelis M, Krabbendam L, Delespaul P, and van Os J (2005): Subtle fluctuations in psychotic phenomena as functional states of abnormal dopamine reactivity in individuals at risk. Biol Psychiatry 58:105-10.

89. Chen J, Lipska BK, Halim N, Ma QD, Matsumoto M, Melhem S, et al. (2004): Functional analysis of genetic variation in catechol-O-methyltransferase (COMT): effects on mRNA, protein, and enzyme activity in postmortem human brain. Am J Hum Genet 75:807-21.

90. Montag C, Buckholtz JW, Hartmann P, Merz M, Burk C, Hennig J, et al. (2008): COMT genetic variation affects fear processing: psychophysiological evidence. Behav Neurosci 122:901-9.

91. Mandelli L, Serretti A, Marino E, Pirovano A, Calati R, and Colombo C(2007): Interaction between serotonin transporter gene, catechol-O-methyltransferase gene and stressful life events in mood disorders. Int J Neuropsychopharmacol 10:437-47.

92. Simons CJ, Wichers M, Derom C, Thiery E, Myin-Germeys I, Krabbendam L, et al. (2009): Subtle gene-environment interactions driving paranoia in daily life. Genes Brain Behav 8:5-12. 
Chapter I 
${ }^{1}$ Department of Psychiatry and Neuropsychology, South Limburg

Mental Health Research and Teaching Network, EURON, Maastricht University, PO Box 616, 6200 MD Maastricht, The Netherlands

${ }^{2}$ Section of Social Cognition, Mondriaan Zorggroep, Heerlen, The Netherlands

${ }^{3}$ School of Psychological Sciences, University of Manchester, UK

${ }^{4}$ Division of Psychological Medicine, Institute of Psychiatry, London, UK 


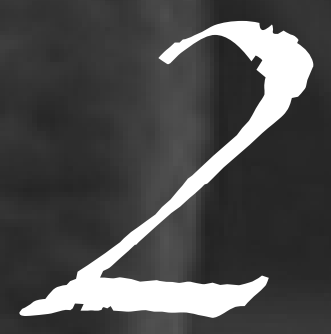

Does the Concept of "Sensitization" Provide a Plausible Mechanism for the Putative Link Between the Environment and Schizophrenia?

Dina Collip ${ }^{1}$, Inez Myin-Germeys ${ }^{1-3}$, and Jim Van Os ${ }^{1,4}$ 


\begin{abstract}
Previous evidence reviewed in Schizophrenia Bulletin suggests the importance of a range of different environmental factors in the development of psychotic illness. It is unlikely, however, that the diversity of environmental influences associated with schizophrenia can be linked to as many different underlying mechanisms. There is evidence that environmental exposures may induce, in interaction with (epi)genetic factors, psychological or physiological alterations that can be traced to a final common pathway of cognitive biases and/or altered dopamine neurotransmission, broadly referred to as "sensitization", facilitating the onset and persistence of psychotic symptoms. At the population level, the behavioral phenotype for sensitization may be examined by quantifying, in populations exposed to environmental risk factors associated with stress or dopamine-agonist drugs, i) the increased rate of persistence (indicating lasting sensitization) of normally transient developmental expressions of subclinical psychotic experiences and ii) the subsequent increased rate of transition to clinical psychotic disorder.
\end{abstract}

Key words: environment/psychosis/schizophrenia/mechanisms/mediators 


\section{Introduction}

In previous issues of Schizophrenia Bulletin [1-9], evidence has been presented suggesting that environmental risk factors (prenatal stress/malnutrition/infection/hypoxia, paternal age, developmental trauma, urbanicity, cannabis, ethnic minority group, social fragmentation) may be associated with an increased risk for schizophrenia and psychotic symptoms, resulting in widespread geographical variation in incidence and prevalence [10]. These studies have several themes in common and are summarized in the box. It is clear that much remains to be clarified. For example, although the research on individual environmental risk factors is mostly consistent, effect sizes have been derived from bias and confounding-prone observational research, none of the hypothesized factors are likely necessary or sufficient, nor are they specific for psychosis outcomes. In addition, variables such as ethnic group, urbanicity or prenatal maternal malnutrition merely represent proxies for one or more non-genetic factors that remain to be identified.

Given these uncertainties, relatively little attention has been paid to what arguably constitutes the most challenging issue: is there any evidence for a plausible mechanism linking exposure to the environment to psychosis outcomes? How does the environment induce change in human beings so that enduring risk states or psychopathological outcomes may result? Rutter [11] has argued that there a number of ways in which the environment can impact on the individual to increase the risk for psychopathology, including effects on gene expression, effects on developmental programming of the brain, effects on neuroendocrine and neurotransmitter functioning, effects on patterns of interpersonal interactions that may shape risk for later psychopathology, and effects on affective and cognitive processing. Therefore, one way to validate the hypothesis of a link between the environment and psychosis is to examine to what degree the two may be plausibly linked by any of the above mentioned cognitive or biological mechanisms. Given the fact that i) it is unlikely that the extreme diversity of environmental influences associated with schizophrenia can be linked to as many different underlying mechanisms and ii) it has been suggested that environmental exposures in schizophrenia may induce psychological or physiological alterations that can be traced to a final common pathway of cognitive biases and/ or altered dopamine neurotransmission [12-15], this particular suggestion will be examined in more detail, subsumed under the broad header of "sensitization".

\section{Sensitization}

Sensitization refers to the observation that individuals who are exposed repeatedly to an environmental risk factor may develop progressively greater responses over time, finally resulting in a lasting change in response amplitude. It has been hypothesized that the process of sensitization is the substrate for the susceptibility to the psychosis-inducing effects of stress and dopamine-agonist drugs observed in patients with schizophrenia $[13,16]$. Given the fact that many environmental exposures associated 
with schizophrenia may be linked to stress or dopamine-agonist drugs, sensitization may represent a common mechanism linking multiple environmental influences.

\section{Sensitization: cognitive and affective processing}

Although the precise exposure under most environmental risk factors associated with schizophrenia remains unknown, many, including trauma, ethnic minority group, urbanicity and social fragmentation can be plausibly traced to "social defeat"-type psychological effects [17] in the realm of interpersonal interactions. Such effects play a key role in recent cognitive models of psychosis [12, 18-23]. Early exposure to environmental risk factors such as developmental trauma and discrimination may shape specific negative beliefs about the self and about others. It has been hypothesized that such negative schemas and self-beliefs may predispose individuals to employ external attributions for negative events, possibly in order to protect the self from negative self-evaluative beliefs (delusion-as-a-defense theory) $[18,24,25]$. Indeed, studies have confirmed that psychotic individuals likely attribute negative events to external causes [18]. Accordingly, dysfunctional beliefs and schemas may moderate the psychotogenic effect of later environmental risk factors, such as adverse life events, for psychosis. It has been argued that stressful events trigger particular emotional and cognitive changes, including automatic cognitive processes and maladaptive conscious appraisals. These, in combination with cognitive biases induced by earlier exposures, may be crucial in the formation and maintenance of positive symptoms of psychosis $[12,26]$. Recent work examining these hypotheses has yielded some evidence that the path from childhood abuse or trauma to adult psychopathology and respectively psychosis is mediated by disrupted self-representations and negative beliefs about others, as well as by alterations in meta-cognition that may predispose to psychosis [27-29]. With the advent of sophisticated virtual reality (VR) techniques, researchers now can conduct controlled experiments in order to study, for example, how paranoia may arise from cognitive biases by observing how people interact with one another and interpret interpersonal behavior in controlled social situations. Early results show that people with paranoid thinking patterns interpret social signals abnormally [30]. These VR experiments can be extended to test whether certain environmental exposures, or indeed certain genotypes, are associated with psychotic interpretations. If ethically acceptable, similar controlled experiments can be conducted using actual environmental exposures. For example, a recent experimental study showed that in individuals with a liability for psychosis, paranoid thoughts were exacerbated by a deprived urban environment [31].

\section{Sensitization: behavioral stress-sensitivity}

It has been demonstrated that persons with a higher than average liability to psychosis are over-reactive to small stressors displaying an exaggerated affective response [32] and increases in the intensity of subtle psychotic experiences associated with minor stresses in the flow of daily life [33]. This effect 
may be described as behavioral sensitization, as it has been shown to result, at least in part, from a sensitization process by which previous exposures to severe stress, such as childhood trauma [34] or stressful life events [35], increase the sensitivity to small stresses in daily life, the cumulative impact of which might lead to the development of impairment and need for care [36]. These findings therefore suggest that the effects of early stress may give rise to a lasting liability in the form of emotional and psychotic reactivity.

\section{Sensitization: dopamine neurotransmission}

Research has revealed that dopamine is released in response to stress in both animals [37-39] and humans [40,41], although not all studies agree [42]. As dopamine is often regarded as the final common pathway of the factors involved in the causation of psychosis $[13,43]$, it is attractive to speculate that dopamine plays a role in the pathway from environmental risk exposure to psychosis.

In animals, there is compelling evidence that disruptions in postnatal rearing conditions can lead to profound and lasting changes in the responsiveness of mesocorticolimbic dopamine neurons to stress and psychostimulants [44-46]. A similar mechanism may exist in humans as well and provide an explanation for differential dopamine reactivity in those with and without psychosis liability $[47,48]$. For example, a recent human study reported that mesolimbic dopamine release in response to psychosocial stress depended on low early life maternal care [41]. In rodents, there is evidence that not only early life stress, but also use of agonist drugs may induce sensitization of dopamine systems [45]. Not only is there evidence for similar mechanisms in humans [49], studies also indicate that schizophrenia is associated with increased amphetamine-induced dopamine release [50].

While these findings go some way towards validating the link between environmental exposures and psychosis, the precise mechanisms and neurocircuitry underlying stress-dopamine interactions and sensitization in schizophrenia remain unknown. There is evidence that mesocortical dopaminergic innervation of the prefrontal cortex (PFC) may regulate the activity of mesolimbic subcortical DA innervations, and that the impact of environmental risk factors may result in taking the PFC "off line", resulting in altered responsiveness of subcortical dopaminergic innervations [51]. Thus, when dopamine transmission is increased by exposure to stress or agonist drugs, there may be a shift in the balance of the system away from prefrontal cortical control and toward limbic predominance, facilitating the onset of psychotic symptoms [45, 52]. Genetic variation may act synergistically with environmental risk factors in shifting the balance between mesocortical and mesolimbic dopamine neurotransmission, explaining interactions between, for example, cannabis and the COMTval158met polymorphism [53, 54]. Other models for gene-environment interactions have been proposed. For example, it has been suggested that excessive levels of catecholamine release during stress impair PFC cognitive function through intracellular signalling pathways; schizophrenia may arise in individuals with mutations in DISC1 and RGS4, who may have weaker regulation of these intra-cellular stress pathways [55]. Other factors may also play a role. For example, there is animal evidence that glucocorticoids may control stress- 
induced sensitization by changing the sensitivity of the mesencephalic dopaminergic transmission to drugs of abuse [56]. Similarly, prenatal stress, associated with schizophrenia [57], may induce changes in dopamine sensitivity of the nucleus accumbens and in the capacity to develop amphetamine-induced sensitization in adulthood, which may be mediated by impaired control of corticosterone secretion in the prenatally-stressed animal [58]. Finally, neurotransmitter sensitisation may be associated with epigenetic mechanisms. Epigenetic factors are inherited and acquired modifications of DNA (eg DNA methylation) and histones that occur without a change in nuclear DNA sequence but may impact on gene expression. The epigenetic state of a gene may be influenced by stress and drugs amongst other things and thus be considered a priori as an important factor mediating environment-schizophrenia relationships [59]. Many studies have identified changes in mRNA levels in key areas involved in dopaminergic neurotransmission including the ventral tegmental area and the nucleus accumbens induced by dopamine-agonist drugs (including cannabis) [60-63]. Similarly, an epigenetic mechanism has been shown to mediate the relationship between variations in mother-infant interactions and the development of individual differences in behavioral and endocrine responses to stress in adulthood [64].

\section{Sensitization: epidemiological predictions}

If environmental risk factors are causally associated with psychotic disorder and sensitization is the mechanism linking risk and outcome, then the challenge is to find a way to measure its behavioral phenotype and demonstrate epidemiological evidence that matches the hypothesis. Cougnard and colleagues [65] suggested that the behavioral phenotype for sensitization may be examined at the population level by quantifying, in populations exposed to environmental risk factors associated with stress or dopamine-agonist drugs, i) the increased rate of persistence (indicating lasting sensitization) of normally transient [66-68] developmental expressions of subclinical psychotic experiences and ii) the subsequent increased rate of transition to clinical psychotic disorder (Fig. 1). In two large, prospective independent general population studies (NEMESIS, $n=7076$ and EDSP, $n=3021$ ) they examined the hypothesis that relatively common, subclinical developmental psychotic experiences would become abnormally persistent when synergistically combined with developmental exposures that may impact on sensitisation such as cannabis, developmental trauma and urbanicity. The authors found that the threeyear persistence rates of psychotic experiences were low at 26\% in NEMESIS and 31\% in EDSP. However, persistence rates were progressively higher with greater baseline number of environmental exposures in predicting follow-up psychotic experiences. The authors concluded that level of environmental risk combines synergistically with subclinical developmental expression of psychosis to cause abnormal persistence, reflecting a mechanism of sensitization.

In a subsequent study, Dominguez and colleagues [69] went one step further and examined the hypothesis that the probability of poor outcome (in the sense of clinical psychotic disorder) of the normally transitory developmental expression of subclinical psychosis in the general population 
becomes progressively greater with more tendency to persistence over time. Expression of psychosis was assessed four times (TO-T3) over a period of 8.4 years in a sample of 845 adolescents from the general population. Transition from subclinical psychosis at T0-T2 to clinical psychosis at T3 was examined as a function of the level of prior persistence of the subclinical phenotype over T0-T2 (subclinical psychosis present never, once, twice or thrice at T0, T1 and T2). The authors found that the more subclinical psychosis persisted over the period T0-T2, the greater the risk of transition to clinical psychosis at T3 in a dose-response fashion.

\section{Conclusion}

Although many questions remain, there is some evidence that environmental exposures may induce, in interaction with (epi)genetic factors, psychological or physiological alterations that can be traced to a final common pathway of cognitive biases and/or altered dopamine neurotransmission, broadly referred to as "sensitization", facilitating the onset and persistence of psychotic symptoms. The behavioral phenotype for sensitization may be examined at the population level by quantifying, in populations exposed to environmental risk factors associated with stress or dopamine-agonist drugs, i) the increased rate of persistence (indicating lasting sensitization) of normally transient developmental expressions of subclinical psychotic experiences and ii) the subsequent increased rate of transition to clinical psychotic disorder. 
Box 1: Common themes of recent reviews on the link between the environment and schizophrenia

1. Most findings are derived from observational studies that can never conclusively exclude bias and confounding

2. It is not clear to what degree genetic liability for psychosis is the origin of the environmental influence (eg psychosis liability contributing to later cannabis use or maternal psychosis liability giving rise to pregnancy complications).

3. For the majority of environmental risk factors, the increase in risk is associated with exposure before adulthood suggesting an interaction with developmental processes. For example, the risk-increasing effects of urbanicity, trauma and cannabis use are limited to exposure during childhood and adolescence, suggesting they may create enduring liabilities that, in interaction with other factors, bring about psychotic disorder in adulthood.

4. Relative risks associated with environmental exposures are modest and none are likely necessary or sufficient. Indeed, for most, interactions with genetic risk factors are hypothesized and some gene-environment interactions using direct or indirect measures of genes and environments have been reported.

5. Many, if not most, of the environmental candidate factors represent proxies for as yet unidentified non-genetic effects. For example, while ethnic group and urbanicity can be readily used as categorical variables in statistical analyses, it is not known what underlying true environmental effect they may represent.

6. The evidence for a link between the environment and psychosis is for many risk factors, at least where this has been studied, not limited to the narrow syndrome of schizophrenia, but extending to broader psychometric expressions of psychosis in the form of psychotic-like symptoms or schizotypy.

7. None of the reported risk factors can claim a specific link to psychosis - all have been associated with a range of other psychiatric and somatic disorders 
Figure 1. Sensitization behavioural phenotype. Person A has "normal" developmental expression of subclinical psychotic experiences that are mild and transient. Person B has similar expression, but longer persistence due to additional but mild environmental exposure. Person $C$ has prolonged persistence due to severe repeated environmental exposure and subsequent transition to clinical psychotic disorder

Level of

psychosis

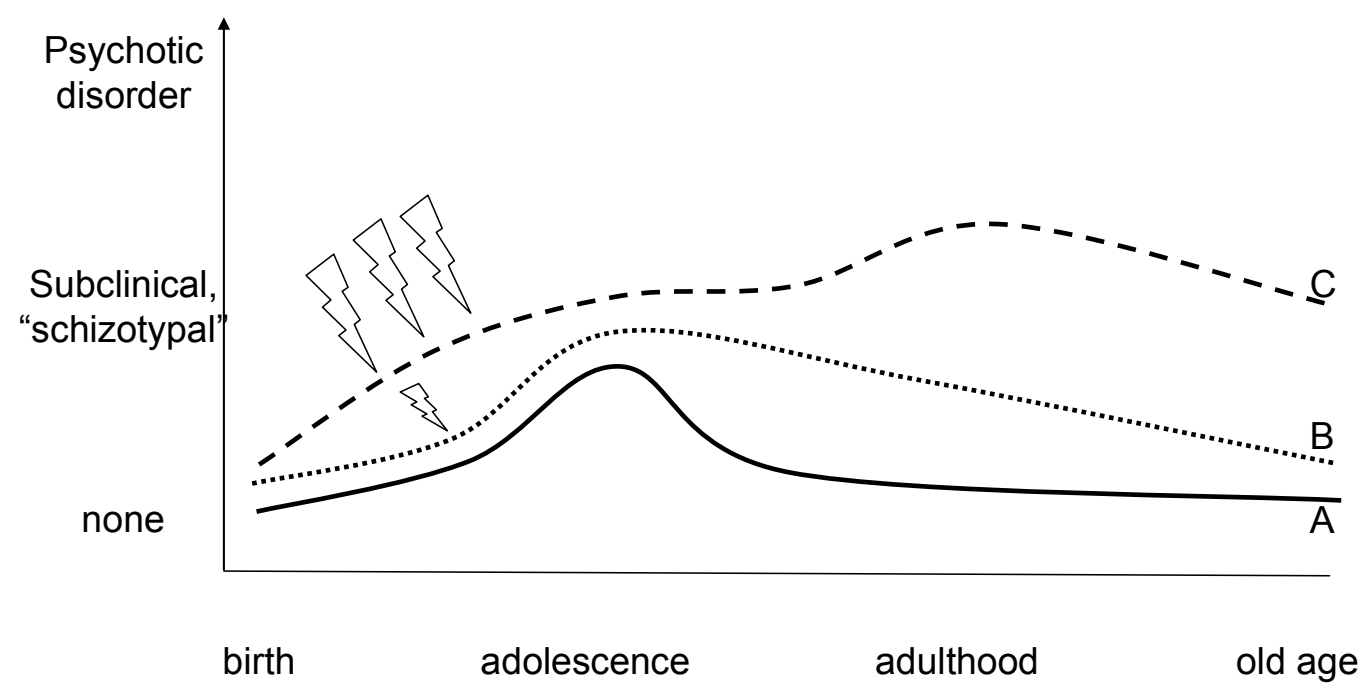




\section{References}

1. Henquet C, Murray R, Linszen D, and van Os J (2005): The environment and schizophrenia: the role of cannabis use. Schizophrenia Bulletin 31:608-612.

2. Morgan C and Fisher H (2007): Environmental factors in schizophrenia: childhood trauma - a critical review. Schizophrenia Bulletin 33:3-10.

3. Fearon P and Morgan C (2006): Environmental factors in schizophrenia: the role of migrant studies. Schizophrenia Bulletin 32:405-408

4. $\quad$ Allardyce J and Boydell J (2006): Review: the wider social environment and schizophrenia. Schizophr Bull 32:592598.

5. Clarke MC, Harley M, and Cannon M (2006): The role of obstetric events in schizophrenia. Schizophrenia Bulletin 32:3-8.

6. Krabbendam L and van Os J (2005): Schizophrenia and urbanicity: a major environmental influence - conditional on genetic risk. Schizophrenia Bulletin 31:795-799.

7. Zammit S, Lewis S, Gunnell D, and Smith GD (2007): Schizophrenia and neural tube defects: comparisons from an epidemiological perspective. Schizophr Bull 33:853-8.

8. Brown AS (2006): Prenatal infection as a risk factor for schizophrenia. Schizophr Bull 32:200-2.

9. Perrin MC, Brown AS, and Malaspina D (2007): Aberrant epigenetic regulation could explain the relationship of paternal age to schizophrenia. Schizophr Bull 33:1270-3.

10. McGrath JJ (2006): Variations in the incidence of schizophrenia: data versus dogma. Schizophrenia Bulletin 32:195197.

11. Rutter M (2005): How the environment affects mental health. British Journal of Psychiatry 186:4-6.

12. Garety PA, Kuipers E, Fowler D, Freeman D, and Bebbington PE (2001): A cognitive model of the positive symptoms of psychosis. Psychol Med 31:189-95.

13. Howes OD, McDonald C, Cannon M, Arseneault L, Boydell J, and Murray RM (2004): Pathways to schizophrenia: the impact of environmental factors. The International Journal of Neuropsychopharmacology 7:S7-S13.

14. Selten J-P and Cantor-Graaf E (2005): Social defeat: risk factor for schizophrenia? The British Journal of Psychiatry 187:101-102.

15. Myin-Germeys I and van Os J (2007): Stress-reactivity in psychosis: evidence for an affective pathway to psychosis. Clinical Psychology Review 27:409-424.

16. Lieberman JA, Sheitman BB, and Kinon BJ (1997): Neurochemical sensitization in the pathophysiology of schizophrenia: deficits and dysfunction in neuronal regulation and plasticity. Neuropsychopharmacology 17:20529.

17. Bjorkqvist K (2001): Social defeat as a stressor in humans. Physiol Behav 73:435-42.

18. Bentall RP and Taylor JL (2006): Psychological processes and paranoia: implications for forensic behavioral science. Behavioural Sciences and the Law 24:277-294.

19. Fowler D, Freeman D, Smith B, Kuipers E, Bebbington P, Bashforth H, et al. (2006): The Brief Core Schema Scales (BCSS): psychometric properties and associations with paranoia and grandiosity in non-clinical and psychosis samples. Psychol Med 36:749-59.

20. Freeman D (2007): Suspicious minds: the psychology of persecutory delusions. Clin Psychol Rev 27:425-57.

21. Hacker D, Birchwood M, Tudway J, Meaden A, and Amphlett C (2007): Acting on voices: omnipotence, sources of threat, and safety-seeking behaviours. Br J Clin Psychol.

22. Morrison AP, Wells A, and Nothard S (2002): Cognitive and emotional predictors of predisposition to hallucinations in non-patients. Br J Clin Psychol 41:259-70. 
23. Peters ER, Joseph SA, and Garety PA (1999): Measurement of delusional ideation in the normal population: introducing the PDI (Peters et al. Delusions Inventory). Schizophr Bull 25:553-76.

24. Freeman D (2007): Suspicious minds: the psychology of persecutory delusions. Clinical Psychology Review 27:425457.

25. Garety PA and Freeman D (1999): Cognitive approaches to delusions: a critical review of theories and evidence. British Journal of Clinical Psychology 38:113-154.

26. Garety PA, Bebbington P, Fowler D, Freeman D, and Kuipers E (2007): Implications for neurobiological research of cognitive models of psychosis: a theoretical paper. Psychol Med 37:1377-91.

27. Finzi-Dottan R and Karu T (2006): From emotional abuse in childhood to psychopathology in adulthood. The Journal of Nervous and Mental Disease 194:616-621.

28. Gracie A, Freeman D, Green S, Garety PA, Kuipers E, Hardy A, et al. (2007): The association between traumatic experience, paranoia and hallucinations: a test of the predictions of psychological models. Acta Psychiatrica Scandinavica 116:280-289.

29. Bak M, Krabbendam L, Janssen I, de Graaf R, Vollebergh W, and van Os J (2005): Early trauma may increase the risk for psychotic experiences by impacting on emotional response and perception of control. Acta Psychiatr Scand 112:360-6.

30. Freeman D, Garety PA, Bebbington P, Slater M, Kuipers E, Fowler D, et al. (2005): The psychology of persecutory ideation II: a virtual reality experimental study. J Nerv Ment Dis 193:309-15.

31. Ellett L, Freeman D, and Garety PA (2007): The psychological effect of an urban environment on individuals with persecutory delusions: The Camberwell walk study. Schizophr Res.

32. Myin-Germeys I, Van Os J, Schwartz JE, Stone AA, and Delespaul PA (2001): Emotional reactivity to daily life stress in psychosis. Arch Gen Psychiatry 58:1137-44.

33. Myin-Germeys I, Marcelis M, Krabbendam L, Delespaul P, and van Os J (2005): Subtle fluctuations in psychotic phenomena as functional states of abnormal dopamine reactivity in individuals at risk. Biol Psychiatry 58:105-10.

34. Glaser JP, van Os J, Portegijs PJ, and Myin-Germeys I (2006): Childhood trauma and emotional reactivity to daily life stress in adult frequent attenders of general practitioners. J Psychosom Res 61:229-36.

35. Myin-Germeys I, Peeters F, Havermans R, Nicolson NA, DeVries MW, Delespaul P, et al. (2003): Emotional reactivity to daily life stress in psychosis and affective disorder: an experience sampling study. Acta Psychiatr Scand 107:12431.

36. Myin-Germeys I and van Os J (2007): Stress-reactivity in psychosis: Evidence for an affective pathway to psychosis. Clin Psychol Rev.

37. Abercrombie ED, Keefe KA, DiFrischia DS, and Zigmond MJ (1989): Differential effect of stress on in vivo dopamine release in striatum, nucleus accumbens, and medial frontal cortex. Journal of Neurochemistry 52:1655-1658.

38. Tidey JW and Miczek KA (1996): Social defeat stress selectively alters medocorticolimbic release: an in vivo microdialysis study. Brain Research 721:140-149.

39. Gresch PJ, Sved AF, Zigmond MJ, and Finlay JM (1994): Stress-induced sensitization of dopamine and norepinephrine efflux in medial prefrontal cortex of the rat. $J$ Neurochem 63:575-83.

40. Wand GS, Oswald LM, McCaul ME, Wong DF, Johnson E, Zhou Y, et al. (2007): Association of amphetamine-induced striatal dopamine release and cortisol responses to psychological stress. Neuropsychopharmacology 32:2310-20.

41. Pruessner JC, Champagne F, Meaney MJ, and Dagher A (2004): Dopamine release in response to a psychological stress in humans and its relationship to early life maternal care: a positron emission tomography study using [11C]raclopride. The Journal of Neuroscience 24:2825-2831.

42. Montgomery AJ, Mehta MA, and Grasby PM (2006): Is psychological stress in man associated with increased striatal dopamine levels?: A [11C]raclopride PET study. Synapse 60:124-31.

43. Kapur S (2003): Psychosis as a state of aberrant salience: a framework linking biology, phenomenology, and 
pharmacology in schizophrenia. The American Journal of Psychiatry 160:13-24.

44. Brake WG, Zhang TY, Diorio J, Meaney MJ, and Gratton A (2004): Influence of early postnatal rearing conditions on mesocorticolimbic dopamine and behavioural responses to psychostimulants and stressors in adult rats. Eur $\mathrm{J}$ Neurosci 19:1863-74.

45. Pani L, Porcella A, and Gessa GL (2000): The role of stress in the pathophysiology of the dopaminergic system. Mol Psychiatry 5:14-21.

46. Hall FS, Wilkinson LS, Humby T, and Robbins TW (1999): Maternal deprivation of neonatal rats produces enduring changes in dopamine function. Synapse 32:37-43.

47. Myin-Germeys I, Marcelis M, Krabbendam L, Delespaul P, and van Os J (2005): Subtle fluctuations in psychotic phenomena as functional states of abnormal dopamine reactivity in individuals at risk. Biological Psychiatry 58.

48. Soligman A, O’Driscoll GA, Pruessner JC, Holahan AL, Boileau I, Gagnon D, et al. (2007): Stress-induced dopamine release in humans at risk of psychosis: a [(11)C] raclopride PET study. Neuropsychopharmacology [Epub ahead of print].

49. Boileau I, Dagher A, Leyton M, Gunn RN, Baker GB, Diksic M, et al. (2006): Modeling sensitization to stimulants in humans: an [11C]raclopride/positron emission tomography study in healthy men. Arch Gen Psychiatry 63:138695.

50. Laruelle M (2000): The role of endogenous sensitization in the pathophysiology of schizophrenia: implications from recent brain imaging studies. Brain Research Reviews 31:371-384.

51. Deutch AY, Clark WA, and Roth RH (1990): Prefrontal cortical dopamine depletion enhances the responsiveness of mesolimbic dopamine neurons to stress. Brain Res 521:311-5.

52. Goto Y, Otani S, and Grace AA (2007): The Yin and Yang of dopamine release: a new perspective. Neuropharmacology 53:583-7.

53. Caspi A, Moffitt TE, Cannon M, McClay J, Murray R, Harrington H, et al. (2005): Moderation of the effect of adolescentonset cannabis use on adult psychosis by a functional polymorphism in the catechol-O-methyltransferase gene: longitudinal evidence of a gene $\mathrm{X}$ environment interaction. Biol Psychiatry 57:1117-27.

54. Henquet C, Rosa A, Krabbendam L, Papiol S, Fananas L, Drukker M, et al. (2006): An Experimental Study of CatecholO-Methyltransferase Val(158)Met Moderation of Delta-9-Tetrahydrocannabinol-Induced Effects on Psychosis and Cognition. Neuropsychopharmacology online publication 16936704.

55. Arnsten AF (2007): Catecholamine and second messenger influences on prefrontal cortical networks of "representational knowledge": a rational bridge between genetics and the symptoms of mental illness. Cereb Cortex 17 Suppl 1:i6-15.

56. Deroche V, Marinelli M, Maccari S, Le Moal M, Simon H, and Piazza PV (1995): Stress-induced sensitization and glucocorticoids. I. Sensitization of dopamine-dependent locomotor effects of amphetamine and morphine depends on stress-induced corticosterone secretion. J Neurosci 15:7181-8.

57. Van Os J and Selten J-P (1998): Prenatal exposure to maternal stress and subsequent schizophrenia: The May 1940 invasion of The Netherlands. British Journal of Psychiatry 172:324-326.

58. Sorg BA and Kalivas PW (1991): Effects of cocaine and footshock stress on extracellular dopamine levels in the ventral striatum. Brain Res 559:29-36.

59. Tsankova N, Renthal W, Kumar A, and Nestler EJ (2007): Epigenetic regulation in psychiatric disorder. Nature Reviews 8:355-367.

60. Freeman WM, Brebner K, Lynch WJ, Patel KM, Robertson DJ, Roberts DC, et al. (2002): Changes in rat frontal cortex gene expression following chronic cocaine. Brain Res Mol Brain Res 104:11-20.

61. McClung CA and Nestler EJ (2003): Regulation of gene expression and cocaine reward by CREB and DeltaFosB. Nat Neurosci 6:1208-15.

62. Villares J (2007): Chronic use of marijuana decreases cannabinoid receptor binding and mRNA expression in the human brain. Neuroscience 145:323-34. 
63. Yao WD, Gainetdinov RR, Arbuckle MI, Sotnikova TD, Cyr M, Beaulieu JM, et al. (2004): Identification of PSD-95 as a regulator of dopamine-mediated synaptic and behavioral plasticity. Neuron 41:625-38.

64. Weaver IC, Cervoni N, Champagne FA, D’Alessio AC, Sharma S, Seckl JR, et al. (2004): Epigenetic programming by maternal behavior. Nat Neurosci 7:847-54.

65. Cougnard A, Marcelis M, Myin-Germeys I, De Graaf R, Vollebergh W, Krabbendam L, et al. (2007): Does normal developmental expression of psychosis combine with environmental risk to cause persistence of psychosis? A psychosis proneness-persistence model. Psychol Med 37:513-27.

66. Hanssen M, Bak M, Bijl R, Vollebergh W, and van Os J (2005): The incidence and outcome of subclinical psychotic experiences in the general population. Br J Clin Psychol 44:181-91.

67. Wiles NJ, Zammit S, Bebbington P, Singleton N, Meltzer H, and Lewis G (2006): Self-reported psychotic symptoms in the general population: results from the longitudinal study of the British National Psychiatric Morbidity Survey. $\mathrm{Br}$ J Psychiatry 188:519-26.

68. Poulton R, Caspi A, Moffitt TE, Cannon M, Murray R, and Harrington H (2000): Children's self-reported psychotic symptoms and adult schizophreniform disorder: a 15-year longitudinal study. Arch Gen Psychiatry 57:1053-8.

69. Dominguez $\mathrm{M}$, Wichers $\mathrm{M}$, Lieb R, Wittchen $\mathrm{H}-\mathrm{U}$, and van Os J (submitted): Evidence that onset of clinical psychosis is the outcome of progressively more persistent subclinical psychotic experiences: an 8-year cohort study. 
${ }^{1}$ Dept of Psychiatry and Psychology, School of Mental Health and Neuroscience, Maastricht University Medical Center, PO Box 616 (DRT 10), 6200 MD Maastricht, the Netherlands

${ }^{2}$ Dept of Interdisciplinary Social Science, University of Utrecht, PO Box 80.140, 3508 TC Utrecht, the Netherlands

${ }^{3}$ Orygen Youth Health Research Centre and Centre for Youth Mental Health, University of Melbourne, 35 Poplar Rd, Parkville, Victoria, 3052, Australia

${ }^{4}$ School of Psychology, University of Birmingham, Edgbaston, Birmingham B15 2TT, UK

${ }^{5}$ King's College London, King's Health Partners, Department of Psychosis Studies, Institute of Psychiatry, De Crespigny Park, London SE5 8AF, UK

*contributed equally 


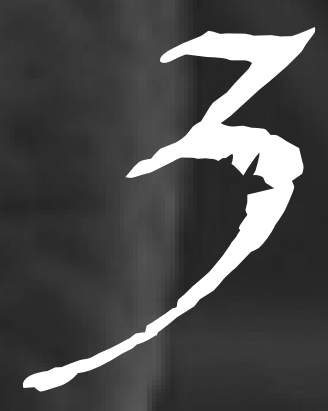

Dynamic association between Interpersonal Functioning \& Positive Symptom Dimensions of P sychosis over Time: a Longitudinal Study of Healthy Adolescents

Dina Collip ${ }^{1 *}$, Johanna T.W. Wigman ${ }^{2 *}$, Ashleigh Lin ${ }^{3,4}$, Barnaby Nelson ${ }^{3}$, Margreet Oorschot ${ }^{1}$, Wilma A.M. Vollebergh ${ }^{2}$, Jaymee Ryan ${ }^{3}$, Gennedy Baksheev ${ }^{3}$, Marieke Wichers ${ }^{1}$, Jim van Os $^{1,5}$, Inez Myin-Germeys ${ }^{1}$, Alison R. Yung ${ }^{3}$ 


\section{Abstract}

\section{Background}

Cross-sectional studies have indicated that alterations in social functioning, particularly interpersonal functioning, are associated with the occurrence of psychotic symptoms and experiences at different levels of the extended psychosis phenotype (ranging from population psychometric expression of liability to overt psychotic disorder). However, more research is needed on the development of this association over time.

\section{Methods}

Cross-lagged path modelling was used to analyse bi-directional, longitudinal associations between four dimensions of subclinical psychotic experiences (persecutory ideation, bizarre experiences, perceptual abnormalities, magical thinking) and interpersonal functioning in an adolescent general population sample ( $\mathrm{N}=881$ at $\mathrm{T} 1, \mathrm{~N}=652$ at $\mathrm{T} 2$ and, $\mathrm{N}=512$ at $\mathrm{T3}$ ), assessed three times in three years.

\section{Results}

All symptom dimensions showed some association with interpersonal functioning over time, but only bizarre experiences and persecutory ideation were consistently and longitudinally associated with interpersonal functioning. Poorer interpersonal functioning predicted higher levels of bizarre experiences and persecutory ideation at later measurement points (both T1 to T2 and T2 to T3).

\section{Conclusions}

Poor interpersonal functioning in adolescence may reflect the earliest expression of neurodevelopmental alterations preceding expression of psychotic experiences, in a symptom-specific fashion.

Key words: psychotic disorder*/interpersonal relation*/social support/longitudinal studies/risk factors/ schizophrenic psychology 


\section{Introduction}

Psychosis is thought to exist as an extended phenotype including levels below clinical expression [1]. Subclinical psychotic experiences are commonly reported in the general population [2], particularly during adolescence, when expression of psychosis proneness peaks [3, 4]. Although mostly transient in nature $[5,6]$, psychotic experiences may be predictive of later psychotic disorder in some individuals $[5,7]$. According to the psychosis proneness - persistence - impairment model [8], the pathway from incidental psychotic experiences to clinical psychosis is mediated by the persistence of these experiences, which may eventually lead to need for care. Persistence of subclinical experiences and shifts along the extended psychosis phenotype are thought to result from an interactive process influenced by a number of biological, psychological and social factors $[9,10]$.

One factor that impacts on risk for psychosis is social functioning. Deviations in social functioning are a common feature in patients with schizophrenia, those experiencing a first episode of psychotic illness, and in help-seeking individuals at ultra-high risk (UHR) for psychotic disorder [11]. This is consistent with evidence of deficits in premorbid social development in childhood [12, 13] and adolescence [14] in individuals destined to develop non-affective psychotic disorder. Individuals at UHR who transition to psychotic disorder also show poorer social functioning compared to those who do not make the transition [15-17] [18]. At the general population level, persistence of subclinical psychotic experiences is similarly associated with poorer social functioning [5]. A recent prospective study of a general population sample from Israel showed that transition from subclinical psychotic experiences to hospital admission for psychotic disorder occurred almost exclusively in the group with psychotic experiences who also showed deviant premorbid social functioning [19].

Together, these findings suggest that poor social functioning, possibly reflecting alterations in neurodevelopment, are associated with development of psychotic illness in those at psychometric risk. However, several issues remain. Most studies have only addressed one direction in the relationship, i.e. social functioning predicting psychosis rather than vice versa, or have used a cross-sectional design, which precludes observing how the variables are related over time. Another issue is that most studies employed a broad and variable definition of social functioning, capturing quality of social relations as well as occupational and educational achievements. Distinguishing between these concepts is important, since they may be differentially related to outcome. For example, Cornblatt and colleagues (2007) showed that poorer social functioning predicted the transition from UHR status to overt psychotic disorder, whereas role (academic/work) functioning did not[15]. Similarly, Velthorst et al. (2010) found that amid a range of disability domains, only "getting along with people" predicted the onset of psychosis in their UHR sample[17].

The concept of subclinical psychotic experiences is similarly broad and captures many dimensions which may be quite different in their pattern of associations with other measures. Several dimensional solutions have been proposed in both adult[20] and adolescent [3, 4] populations. Yung and colleagues (2009) demonstrated a four-dimensional structure of subclinical positive psychotic experiences 
(persecutory ideation, bizarre experiences, perceptual abnormalities and magical thinking). These separate symptom dimensions were differentially associated with secondary distress and other measures of psychopathology such as depression.

Research has shown that environmental risk factors for psychotic experiences may be associated with specific dimensions of these experiences, such as hallucinations or paranoia[21] . It may be hypothesized that distinct symptom dimensions of psychotic experiences may also be differentially related to aspects of interpersonal functioning. For example, persecutory ideation may be related to interpersonal functioning because paranoia frequently involves misinterpretation of other people's behavior [22]. Furthermore, experimental studies using virtual reality paradigms to investigate paranoia in different social contexts have demonstrated that individuals with paranoid thinking patterns interpret neutral social signals as threatening $[23,24]$.

The current study addressed the longitudinal association between dimensions of subclinical positive psychotic experiences (as identified in the current sample by Yung et al., 2009) and interpersonal functioning in an adolescent general population sample followed over three years. The study aimed to examine longitudinal bidirectional associations between interpersonal functioning and positive psychotic experiences. Interpersonal functioning was defined as functioning in context of family and peers, and four specific subdimensions of subclinical psychotic experiences were examined, including, persecutory ideation, bizarre experiences, perceptual abnormalities and magical thinking. Since earlier work has previously found association of social function with depressive and negative symptoms, these symptoms were controlled for[25].

\section{Methods}

Sample

Participants were recruited through secondary schools in the western metropolitan region of Melbourne, Australia. Sixty secondary schools were approached to participate; 34 consented. Three data collection waves were completed: T1 (baseline), $\mathrm{N}=881$; T2, (12 months after baseline); $\mathrm{N}=652$ (74\% of original cohort); and T3 (three years after baseline assessment), $\mathrm{N}=512$ (58\% of the original cohort). At baseline, $51 \%$ was female. The mean age was 15.6 years (SD 2.6).

At T1, students from each school were assessed by questionnaire. Trained research assistants were present in the classroom to answer queries. All participants provided written informed consent and assent from their parent/guardian. The second wave of data collection (T2) comprised a semi-structured interview and questionnaires conducted in the participant's home or at Orygen Youth Health, a youth mental health service. Written consent was again obtained from participants and their parent/guardian if they were still under 18 years of age. This process was repeated atT3. The study was approved by Research and Ethics Committees at the University of Melbourne, Victorian Department of Education and the Catholic Education Office. 


\section{Instruments}

The Community Assessment of Psychic Experiences (CAPE) positive experiences subscale (20 selfreported items), based on the Peters et al Delusion Inventory [26], was used to assess psychotic experiences at all study phases [27]. The CAPE negative symptom subscale was only assessed at T1. Each CAPE item rates two aspects of psychotic experiences: (i) frequency and (ii) associated distress, both rated on a four-point scale of never/not distressed (1); sometimes/a bit distressed (2); often/quite distressed (3); nearly always/very distressed (4). The CAPE items showed good internal consistency at all time points ( $\mathrm{T} 1$, Cronbach's alpha=0.85; $\mathrm{T} 2$ and $\mathrm{T} 3,0.82$ ). The 20 frequency items of the positive experience subscale were used to index psychotic experiences. The four sum scores of the subscales of the CAPE defined by Yung et al. (2009) (persecutory ideation, bizarre experiences, perceptual abnormalities, magical thinking) were used to separately investigate the association of each symptom dimension with interpersonal functioning. Persecutory ideation was defined using 7 items including "Have you ever felt that you are being persecuted in some way?". Bizarre experiences included 6 items including, for example: "Have you ever felt as if the thoughts in your head are being taken away from you?", 3 items measured perceptual abnormalities, for example:" Have you ever heard voices when you were alone?") and 4 items defined magical thinking, such as: "Have you ever thought that people can communicate telepathically?" The sum score of the 12 frequency items of the negative subscale was used as a continuous indicator of baseline subclinical negative symptoms (Cronbach's alpha=0.83 at T1).

The Revised Multidimensional Assessment of Functioning Scale (RMAFS) was used to assess interpersonal functioning at all three measurement points. The 23-item questionnaire designed at Orygen Youth Health assesses functioning in the domains of family, peer and general daily life. Peer relationships were assessed with six items (e.g., "I feel close to my friends", "I spend quite a lot of time with my friends") and family functioning with seven items (e.g., "I get on well with my parents","My parents disapprove of my friends, lifestyle or appearance"). Items are rated from "not at all or rarely applicable" (rated "0") to "(almost) always applicable" (rated " 5 "), higher scores indicating better functioning. Peer functioning and family functioning (both as observed variables) were set to load on a latent factor labelled "interpersonal functioning".

The Centre for Epidemiologic Studies Depression Scale (CES-D) was used at T1 to assess level of selfreported depressive symptomatology over the past week [28]. The CES-D consists of 20 items that rate frequency of depressive symptoms from 1 (rarely) to 4 (mostly). The sum score defined continuous depression outcome (T1, Cronbach's alpha=0.75).

\section{Analysis}

Analysis was conducted with Mplus 5.1 (Muthen \& Muthen, 1998-2007) and PASW Statistics 18. Full Information Maximum Likelihood (FIML) was used for model estimation in Mplus and, given that data 
were non-normally distributed, robust $M L(M L R)$ was used. This method estimates a mean-adjusted $\chi^{2}$ that is robust to non-normality. Baseline differences between participants who completed all assessments and those who dropped out of the study at T2 or T3 were analyzed using independent t-tests.

Path analysis was used to investigate relationships between symptom dimensions of subclinical positive psychotic experiences and interpersonal functioning over time, using observed as well as latent variables. Stability paths of symptom dimensions and interpersonal functioning over time were also included in the model. All dimensions of subclinical psychotic experiences were entered as observed variables. A path analyses was used to examine a model of interpersonal functioning and psychotic experiences.

In the model, both family and peer functioning were used as indicators of the latent factor of interpersonal functioning. This latent factor of interpersonal functioning (one for each measurement point) represents an estimation of a general interpersonal functioning phenotype, i.e. the overlap between interpersonal functioning in both domains. A cross-lagged path model was made with interpersonal functioning and the four CAPE sub-dimensions. Paths were drawn allowing psychotic experiences to predict interpersonal functioning over time and vice versa. All symptom dimension scores (persecutory ideation, bizarre experiences, perceptual abnormalities, magical thinking) and interpersonal functioning were allowed to correlate at all time points. To extract the unique contribution of situation-specific functioning, the model also contains two context-specific common factors, one each for family and peer interpersonal functioning; hence, the specific association between subclinical psychotic experiences and general interpersonal functioning was investigated. This approach, combining both aspects of interpersonal functioning into one latent factor, reduces error variance. The path model was corrected for gender, baseline depression as measured by CES-D and baseline negative symptoms as measured by CAPE at baseline [25].

\section{Results}

\section{Descriptives}

There were no significant differences in age, socio-economic status, baseline depression, overall CAPE scores and interpersonal or general functioning between participants who completed all phases of the study and those who dropped out at T2 or T3. Participants who completed all phases had lower baseline scores on persecutory ideations $(t(868)=-2.02, p=0.044)$ and magical thinking $(t(860)=-2.06$, $\mathrm{p}=0.040$ ) than those who dropped out at T2 or T3. There were no baseline differences between the groups with respect to the other symptom dimensions. Furthermore, we tested cannabis use and socio-economic status as possible confounders; however, although cannabis was associated with psychotic experiences, it was not with social functioning, which would be required for it to confound the association. Furthermore, socio-economic status was associated with interpersonal functioning, but not with psychotic experiences. Therefore these factors were not included in the model. 


\section{Path analysis}

\section{Model with latent interpersonal functioning factor}

The model for interpersonal functioning with all paths included showed good model fit $\left[\chi^{2}(123)=\right.$ 253.543; $C F I=0.962$ and $R M S E A=0.035]$. In Figure 1, all significant paths $(p<.05)$ are shown (beta coefficients indicate path strength). As expected, symptom dimensions were inter-correlated at all different time points (for parsimony, these correlations are not depicted). Cross-sectional correlations between symptoms dimensions were positive (range $r 0.20-0.47$ ). At $\mathrm{T} 1$, there was no significant correlation between interpersonal functioning and bizarre experiences $(r=0.01, p=0.86)$, perceptual abnormalities $(r=-0.09, p=0.11)$, persecutory ideation $(r=-0.05, p=0.41)$, or magical thinking $(r=0.03$, $p=0.65)$. At $T 2$, interpersonal functioning was negatively correlated with bizarre experiences $(r=-0.27$, $p=0.004)$, perceptual abnormalities $(r=-0.18, p=0.04)$ and persecutory ideation $(r=-0.41, p=0.000)$, but not with magical thinking $(r=0.08, p=0.35)$. At $T 3$, interpersonal functioning was correlated with persecutory ideation $(r=-0.35, p=.0 .000)$, but not bizarre experiences $(r=-0.16, p=0.08)$, perceptual abnormalities $(r=-0.15, p=0.10)$ or magical thinking $(r=-0.08, p=0.39)$. The interpersonal functioning factor was indicated by both peer and family functioning. This model showed good model fit and all items loaded significantly $(p<.05)$ on the factor. The factor loadings were quite stable over time: 0.72 0.80 for family functioning and 0.41-0.56 for peer functioning. Factor loadings were higher for family functioning than peer functioning, indicating that the common interpersonal functioning factor was determined more strongly by functioning in family than peer context.

Longitudinally, interpersonal functioning at T1 predicted bizarre experiences $(\beta=-0.15, p=0.014)$ and persecutory ideation $(\beta=-0.17 ; p=0.002)$, but not perceptual abnormalities or magical thinking at $\mathrm{T} 2$. Interpersonal functioning at $\mathrm{T} 2$ predicted bizarre experiences $(\beta=-0.17, p=0.024)$, persecutory ideation $(\beta=-0.17, p=0.017)$, perceptual abnormalities $(\beta=-0.16, p=0.042)$ and magical thinking $(\beta=-0.18, p=0.007)$ at T3. None of the subclinical psychotic experiences predicted interpersonal functioning over time.

\section{Discussion}

The present study investigated the bidirectional, longitudinal association between interpersonal functioning and different dimensions of subclinical positive psychotic experiences (persecutory ideation, bizarre experiences, perceptual abnormalities, magical thinking) in an adolescent general population sample. All sub-dimensions showed some longitudinal association with interpersonal functioning, interpersonal functioning predicting later psychotic experiences, but only bizarre experiences and persecutory ideation were predicted consistently. In addition, while deviations in interpersonal functioning predicted persecutory ideation and bizarre experiences over time, this did not hold the other way round. The unidirectional nature of the association is consistent with earlier research suggesting that poor social functioning precedes the occurrence of symptoms and transition to 
psychosis in individuals with increased risk for psychotic disorder [29]. The finding that magical thinking was less strongly predicted by interpersonal functioning is consistent with earlier work showing that this dimension represents a milder form of psychotic experiences [3, 4]. On the other hand, a less robust association between interpersonal functioning and perceptual abnormalities contrasts previous findings, which suggested that this dimension carries more pathological weight, e.g. shows a strong association with depressive symptoms $[4,30]$. It is possible, however, that the more pathological nature of perceptual abnormalities is mediated by associations with other dimensions of psychopathology (e.g. behavioural or emotional problems) rather than directly by interpersonal functioning [4]. Other research demonstrates that the link between functioning and subclinical perceptual abnormalities emerges mainly when perceptual abnormalities are accompanied by delusional experiences [9], suggesting that a deterioration in social functioning is neither required nor sufficient for subclinical perceptual abnormalities to occur.

The current results extend earlier work in several ways. First, most studies have examined overall psychotic symptomatology or psychotic illness as the outcome, but not separate (subclinical) symptom dimensions [31]. Since different subtypes of psychotic symptoms may be differentially related to outcome [30], it is important to examine subtypes separately. Second, earlier studies comparing social functioning between groups at different levels of risk for psychosis often employed cross-sectional designs. This makes it impossible to draw conclusions about possible causality, in contrast to the present study. Third, most studies tested only one direction of the association, namely whether interpersonal functioning predicts psychosis [18]. This study examined the association bi-directionally. Importantly, the majority of studies were conducted in help-seeking UHR or patient populations, but not in the general population. In help-seeking and clinical populations, it may be difficult to draw conclusions, because the very presence of clinical psychotic experiences may colour the interpretation of (neutral) social stimuli. The current results emphasize the importance of the quality of interpersonal relations in the development of specific psychotic experiences at the level of the general population and suggest that at the subclinical stages of psychosis, interpersonal functioning may be particularly important for the formation of persecutory ideation and bizarre experiences.

Several mechanisms may be hypothesized to underlie this association. First, family interactions are particularly important early in life when the child develops basic schemas about interpersonal relationships, and may determine trust in others from early life onwards[32]. This is supported by the findings that factor loadings of peer functioning on the common interpersonal functioning factor were lower than the factor loadings of family functioning, suggesting that family functioning still plays an important, central role in determining social functioning in adolescence. Early (deficient) interpersonal functioning may thus be a powerful (psychological) mechanism for onset of persecutory ideation. Second, neurodevelopmental mechanisms may drive these longitudinal associations.

The direction of the association between interpersonal functioning and psychotic experiences is in line with earlier work by Dominguez and colleagues (2010), who found in a longitudinal study on adolescents that negative symptoms, that were associated with markers of developmental deviance, preceded 
positive symptoms, but not the other way around[33]. Thus, social dysfunction and negative symptoms preceding positive symptoms may reflect the unfolding of an underlying neurodevelopmental process associated with psychosis liability.

Social functioning is closely related to social cognition[34] and individuals at risk for psychosis show alterations not only in social functioning, but also in social cognition, including poor emotion recognition and altered theory of mind capacities[35,36]. These deviations may increase the probability of psychotic symptom expression, as early subclinical pathological ideas are not corrected in social interaction, resulting in even worse interpersonal functioning [37]. Breaking this cycle may be important and may offer opportunities for early intervention [38], for example using CBT [39].

The results of the study should be interpreted in light of its strengths and limitations. First, clinical outcome data were not available; however, the main aim was to examine the development of symptoms at the level of subclinical expression during a phase of life characterized by both a peak in psychosis proneness and changing social roles and relationships. Second, there was significant sample attrition between T1 and T3. However, there were no differences in either CAPE scores or interpersonal functioning between those who did and did not remain in the study. Furthermore, the estimator used for model estimation used all available data and did not delete individuals list-wise.

Keeping these limitations in mind, this study's major strength was the availability of both symptom dimensions and interpersonal functioning at all measurement points, enabling the testing of bidirectional associations in one model and to address this relationship in more detail. Furthermore, a latent factor of interpersonal functioning was used, reducing error variance and representing a more exact estimator of interpersonal functioning since it represents the overlap between peer and family functioning. Further examination of this kind of longitudinal association between variables early in life may have implications for psychological models of psychosis, help test plausible causal mechanisms underlying shifts along the extended psychosis continuum and development of need for care, and gain a better understanding of early mechanisms preceding the development of subclinical psychotic experiences. It should be remembered that associations between psychotic experiences and other concepts, such as interpersonal functioning, may differ at different positions along the extended psychosis continuum. 
Funding This work was supported by a grant to from the Dutch "Stichting Koningsheide" to Johanna Wigman. Ashleigh Lin is supported by a National Health and Medical Research Council (NHMRC) program grant (566529). Alison Yung is supported by a NHMRC Senior Research Fellowship and a NHMRC program grant (566529). Barnaby Nelson is supported by a NARSAD Young Investigator Award and a Ronald Phillip Griffith Fellowship. This research project was supported by the Colonial Foundation. Inez Myin-Germeys was supported by a 2006 NARSAD Young Investigator award and by the Dutch Medical Council (VENI and VIDI grant). These funding sources had no further role in study design; in the collection, analysis and interpretation of data; in the writing of the report; and in the decision to submit the paper for publication.

Acknowledgements We would like to thank all participants and staff involved in the study. 
Figure 1. Longitudinal associations between interpersonal functioning and symptom dimension scores

Note: Only significant paths are depicted. Latent factors are represented by ovals or circles and manifest (i.e. observed) variables as rectangles. All symptom dimensions correlated at all time points (correlations coefficients between 0.20 and 0.47 ; arrows are not being displayed to keep the figure more simple). Effect sizes are given in beta's. Model is adjusted for gender, subclinical negative symptom scores and depression scores at T1.

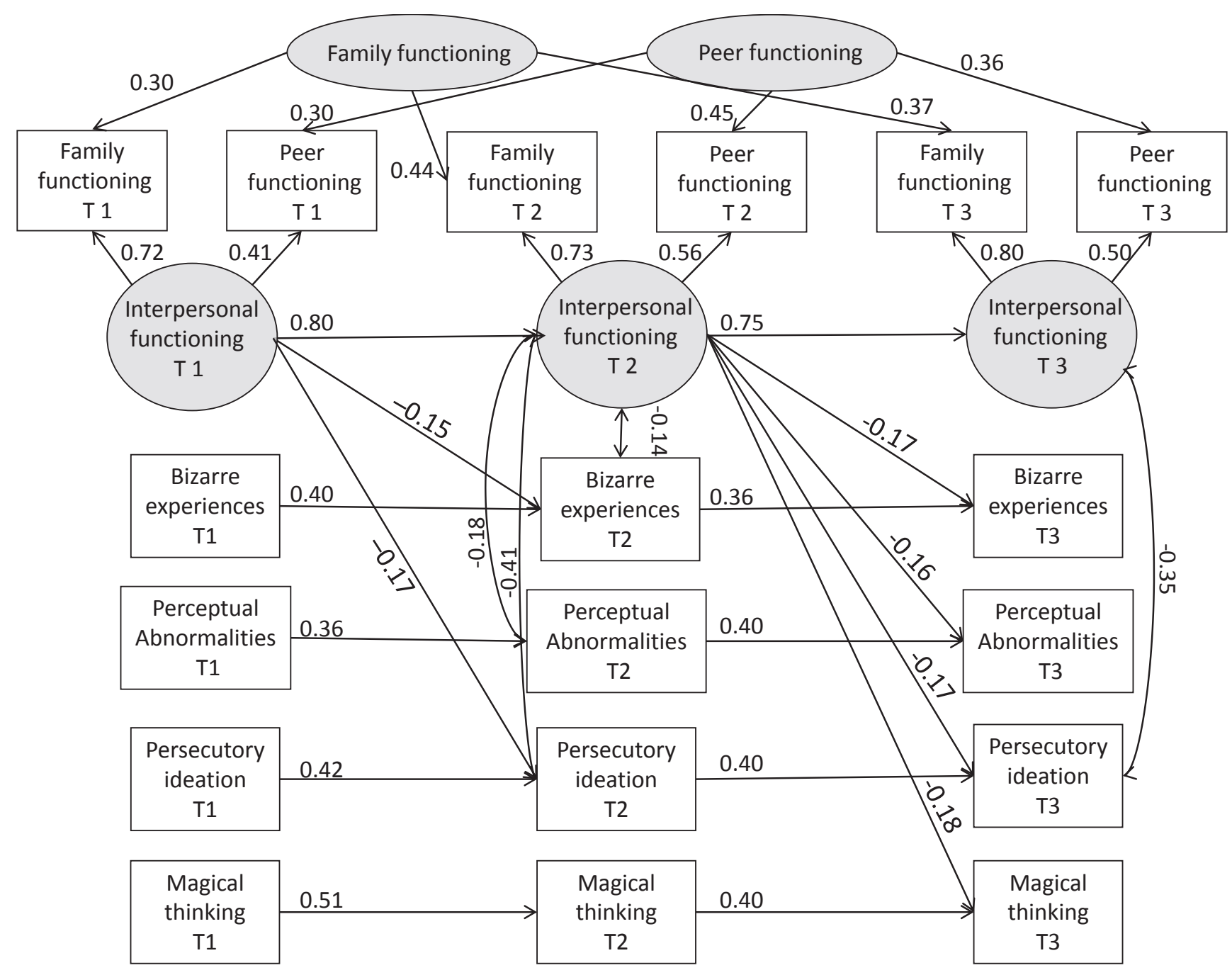




\section{References}

1. Johns LC and van Os J (2001): The continuity of psychotic experiences in the general population. Clin Psychol Rev 21:1125-41.

2. van Os J, Linscott RJ, Myin-Germeys I, Delespaul P, and Krabbendam L (2009): A systematic review and meta-analysis of the psychosis continuum: evidence for a psychosis proneness-persistence-impairment model of psychotic disorder. Psychol Med 39:179-95.

3. Yung AR, Nelson B, Baker K, Buckby JA, Baksheev G, and Cosgrave EM (2009): Psychotic-like experiences in a community sample of adolescents: implications for the continuum model of psychosis and prediction of schizophrenia. Aust N Z J Psychiatry 43:118-128.

4. Wigman JT, Vollebergh WA, Raaijmakers QA, ledema J, van Dorsselaer S, Ormel J, et al. (2009): The Structure of The Extended Psychosis Phenotype in Early Adolescence--A Cross-sample Replication. Schizophr Bull.

5. Dominguez MG, Wichers M, Lieb R, Wittchen HU, and van Os J (2009): Evidence that onset of clinical psychosis is an outcome of progressively more persistent subclinical psychotic experiences: an 8-year cohort study. Schizophr Bull 37:84-93.

6. Wiles NJ, Zammit S, Bebbington P, Singleton N, Meltzer H, and Lewis G (2006): Self-reported psychotic symptoms in the general population: results from the longitudinal study of the British National Psychiatric Morbidity Survey. $B r$ J Psychiatry 188:519-26.

7. Poulton R, Caspi A, Moffitt TE, Cannon M, Murray R, and Harrington H (2000): Children's self-reported psychotic symptoms and adult schizophreniform disorder: a 15-year longitudinal study. Arch Gen Psychiatry 57:1053-8.

8. Cougnard A, Marcelis M, Myin-Germeys I, De Graaf R, Vollebergh W, Krabbendam L, et al. (2007): Does normal developmental expression of psychosis combine with environmental risk to cause persistence of psychosis? $A$ psychosis proneness-persistence model. Psychol Med:1-15.

9. Smeets F, Lataster T, Dominguez MD, Hommes J, Lieb R, Wittchen HU, et al. (2010): Evidence That Onset of Psychosis in the Population Reflects Early Hallucinatory Experiences That Through Environmental Risks and Affective Dysregulation Become Complicated by Delusions. Schizophr Bull.

10. Spauwen J, Krabbendam L, Lieb R, Wittchen HU, and van Os J (2006): Evidence that the outcome of developmental expression of psychosis is worse for adolescents growing up in an urban environment. Psychol Med 36:407-15.

11. Addington J, Penn D, Woods SW, Addington D, and Perkins DO (2008): Social functioning in individuals at clinical high risk for psychosis. Schizophr Res 99:119-124.

12. Cannon M, Caspi A, Moffitt TE, Harrington H, Taylor A, Murray RM, et al. (2002): Evidence for early-childhood, pandevelopmental impairment specific to schizophreniform disorder: results from a longitudinal birth cohort. Arch Gen Psychiatry 59:449-56.

13. Dworkin RH, Cornblatt BA, Friedmann R, Kaplansky LM, Lewis JA, Rinaldi A, et al. (1993): Childhood precursors of affective vs. social deficits in adolescents at risk for schizophrenia. Schizophr Bull 19:563-77.

14. Davidson M, Reichenberg A, Rabinowitz J, Weiser M, Kaplan Z, and Mark M (1999): Behavioral and intellectual markers for schizophrenia in apparently healthy male adolescents. Am J Psychiatry 156:1328-1335.

15. Cornblatt BA, Auther AM, Niendam T, Smith CW, Zinberg J, Bearden CE, et al. (2007): Preliminary findings for two new measures of social and role functioning in the prodromal phase of schizophrenia. Schizophr Bull 33:688-702.

16. Yung AR, McGorry PD, McFarlane CA, Jackson HJ, Patton GC, and Rakkar A (1996): Monitoring and care of young people at incipient risk of psychosis. Schizophr Bull 22:283-303.

17. Velthorst E, Nieman DH, Linszen D, Becker H, de Haan L, Dingemans PM, et al. (2010): Disability in people clinically at high risk of psychosis. Br J Psychiatry 197:278-84.

18. Dragt S, Nieman DH, Veltman D, Becker HE, van de Fliert R, de Haan L, et al. (2010): Environmental factors and social adjustment as predictors of a first psychosis in subjects at ultra high risk. Schizophr Res 125:69-76.

19. Werbeloff N, Drukker M, Dohrenwend B, Levav I, Yoffe R, van Os J, et al. (2009): Self-reported psychotic symptoms in the community are associated with increased risk of later hospitalization for non-affective psychotic disorders. 
Schizophr Bull 35.

20. Stefanis NC, Hanssen M, Smirnis NK, Avramopoulos DA, Evdokimidis IK, Stefanis CN, et al. (2002): Evidence that three dimensions of psychosis have a distribution in the general population. Psychol Med 32:347-358.

21. Bentall RP and Fernyhough C (2008): Social predictors of psychotic experiences: specificity and psychological mechanisms. Schizophr Bull 34:1012-1020.

22. Freeman D (2008): Studying and treating schizophrenia using virtual reality: a new paradigm. Schizophr Bull 34:60510.

23. Ellett L, Freeman D, and Garety PA (2008): The psychological effect of an urban environment on individuals with persecutory delusions: The Camberwell walk study. Schizophr Res.

24. Freeman D, Garety PA, Bebbington P, Slater M, Kuipers E, Fowler D, et al. (2005): The psychology of persecutory ideation II: a virtual reality experimental study. J Nerv Ment Dis 193:309-15.

25. Corcoran CM, Kimhy D, Parrilla-Escobar MA, Cressman VL, Stanford AD, Thompson J, et al. (2011): The relationship of social function to depressive and negative symptoms in individuals at clinical high risk for psychosis. Psychol Med 41:251-61.

26. Peters ER, Joseph SA, and Garety PA (1999): Measurement of delusional ideation in the normal population: introducing the PDI (Peters et al. Delusions Inventory). Schizophr Bull 25:553-76.

27. Konings M, Bak M, Hanssen M, van Os J, and Krabbendam L (2006): Validity and reliability of the CAPE: a self-report instrument for the measurement of psychotic experiences in the general population. Acta Psychiatr Scand 114:5561.

28. Radloff LS (1977): The CES-D Scale: A Self Report Depression Scale for Research in the General Population. Appl Psych Meas 1:385-401.

29. Cannon M, Jones P, Huttunen MO, Tanskanen A, Huttunen T, Rabe-Hesketh S, et al. (1999): School performance in Finnish children and later development of schizophrenia: a population-based longitudinal study. Arch Gen Psychiatry 56:457-463.

30. Yung AR, Buckby JA, Cotton SM, Cosgrave EM, Killackey EJ, Stanford C, et al. (2006): Psychotic-like experiences in nonpsychotic help-seekers: associations with distress, depression, and disability. Schizophr Bull 32:352.

31. Crow TJ (1998): Precursors of psychosis as pointers to the Homo sapiens-specific mate recognition system of language. Br J Psychiatry 172:289-90.

32. Belsky J, Steinberg L, and Draper P (1991): Childhood experience, interpersonal development, and reproductive strategy: An evolutionary theory of socialization. Child Dev 62:647-670.

33. Dominguez MD, Saka MC, Lieb R, Wittchen HU, and van Os J (2010): Early expression of negative/disorganized symptoms predicting psychotic experiences and subsequent clinical psychosis: a 10-year study. Am J Psychiatry 167:1075-82.

34. Pinkham AE and Penn DL (2006): Neurocognitive and social cognitive predictors of interpersonal skill in schizophrenia. Psychiatry Res 143:167-78.

35. Gibson CM, Penn DL, Prinstein MJ, Perkins DO, and Belger A Social skill and social cognition in adolescents at genetic risk for psychosis. Schizophr Res 122:179-84.

36. Brown LA and Cohen AS Facial emotion recognition in schizotypy: the role of accuracy and social cognitive bias. $J$ Int Neuropsychol Soc 16:474-83.

37. Breier A and Strauss JS (1984): The role of social relationships in the recovery from psychotic disorders. Am J Psychiatry 141:949-955.

38. McGorry P, Nordentoft M, and Simonsen E (2005): Introduction to "Early psychosis: a bridge to the future". Br J Psychiatry Suppl 48:s1-3.

39. French P, Shryane N, Bentall RP, Lewis SW, and Morrison AP (2007): Effects of cognitive therapy on the longitudinal development of psychotic experiences in people at high risk of developing psychosis. Br J Psychiatry Suppl 51:s82- 


\footnotetext{
${ }^{1}$ Department of Psychiatry and Neuropsychology, South Limburg Mental Health Research and Teaching Network, EURON, Maastricht University, Maastricht, The Netherlands

${ }^{2}$ Faculty of Psychology, Open University of the Netherlands, Heerlen, The Netherlands

${ }^{3}$ Division of Psychological Medicine, Institute of Psychiatry, London, UK

${ }^{4}$ School of Psychology, University of Bangor, Bangor, UK
} 


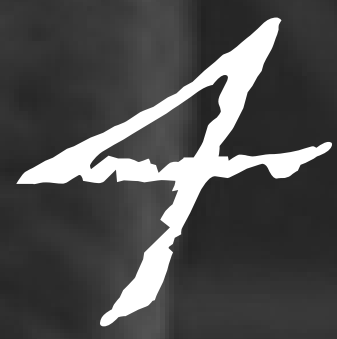

\section{Social World Interactions: \\ How Company Connects to Paranoia}

D. Collip ${ }^{1}$, M. Oorschot ${ }^{1}$, V. Thewissen ${ }^{1,2}$, J. Van Os ${ }^{1,3}$, R. Bentall ${ }^{4}$ and I. Myin-Germeys ${ }^{1}$ 


\section{Abstract}

\section{Background}

Experimental studies have indicated that social contact, even when it is neutral, triggers paranoid thinking in people who score high on clinical or sub-clinical paranoia. We investigated whether contextual variables are predictive of momentary increases in the intensity of paranoid thinking in a sample of participants ranging across a psychometric paranoia continuum.

\section{Methods}

The sample ( $\mathrm{N}=154)$ consisted of 30 currently paranoid patients, 34 currently non-paranoid patients, 15 remitted psychotic patients, 38 high-schizotypy participants, and 37 control subjects. Based on their total score on Fenigstein's Paranoia Scale, three groups with different degrees of paranoia were defined. The Experience Sampling Method (a structured diary technique) was used to assess momentary social context, perceived social threat and paranoia in daily life.

\section{Results}

There were differences in the effect of social company on momentary levels of paranoia and perceived social threat across the range of trait paranoia. The low and medium paranoia group reported higher levels of perceived social threat when with less familiar compared to familiar individuals. The medium paranoia group reported more paranoia in less familiar company. The high paranoia group reported no difference in the perception of social threat or momentary paranoia between familiar and unfamiliar contacts.

\section{Conclusions}

Paranoid thinking is context-dependent in individuals with medium or at-risk levels of trait paranoia. Perceived social threat seems to be context dependent in the low paranoia group. However, at high levels of trait paranoia momentary paranoia and momentary perceived social threat become autonomous and independent of social reality.

Key words: environment/psychosis/mechanism/context/daily life/experience sampling 


\section{Introduction}

A key feature of paranoid ideation, one of the most common delusions in psychosis [1, 2], is a misinterpretation of other people's behavior [3]. Paranoia can be understood as an exaggerated perception or belief that others have malevolent or harmful intentions towards oneself $[4,5]$. Some authors have suggested that aberrant motivational salience of neutral environmental stimuli may be responsible for such beliefs $[6,7]$. The social environment may thus be particularly important in the formation of paranoia.

Several experiments have confirmed the significance of social factors in the development of psychosis, and paranoia in particular. One experiment exposed participants briefly to a deprived urban environment. This real life exposure exacerbated paranoid thinking in individuals with psychosis [8]. Some recent experimental studies used virtual reality to investigate paranoia in different social contexts such as a library or a metro train [8]. Avatars in these virtual reality settings showing ambiguous behavior (looking, smiling, talking) provoked ideas of persecution and reference in individuals from the general population with increased interpersonal sensitivity and high anxiety levels [9]. Moreover, individuals with paranoid thinking patterns abnormally interpreted neutral social signals [10]. These studies thus suggest an important association between the environmental context and paranoia.

These studies as well as some daily life studies have suggested that paranoia may fluctuate in intensity and preoccupation, not only over days or months but even over moments within the day [11]. Changes in social context may be a crucial factor in these short-term fluctuations. Valid tests of whether contextual factors can trigger moment-to-moment variations in paranoia require momentary assessment strategies such as the Experience Sampling Method (ESM) $[12,13]$. ESM is a structured diary technique that captures mental states and contexts in the flow of daily life. ESM has been successfully applied to measure the experience of delusions and hallucinations in the course of everyday life in psychotic disorders [11, 14, 15]. A non-clinical population study by Verdoux et al. (2003) revealed that in individuals with high psychosis vulnerability fluctuations in general psychotic experiences depended on changes in social contacts. Specifically, higher risk of minor psychotic experiences was found in the presence of non-familiar individuals, while lower risk was reported in the presence of family or friends [16]. A study examining the context of delusions in a clinical population reported that the presence of familiar individuals decreased the probability of occurrence of delusions in individuals with a psychotic disorder [11]. These findings suggest that the social environment may influence the intensity of delusional ideations in the flow of daily life. However, specific associations underlying paranoid thinking have not yet been identified.

Paranoia may exist as a continuous trait or phenotype in nature and is thought to be expressed also at levels well below psychotic illness [17], in which case it is usually referred to as psychosis proneness, schizotypy or at-risk mental state [18]. Underlying mechanisms associated with paranoid symptoms are 
also likely to operate in individuals who have an at-risk mental state. Alternatively, there might not only be quantitative but also qualitative differences in the underlying mechanisms of clinical and subclinical paranoia $[17,19,20]$. For instance, two different types of paranoid beliefs, poor me paranoia (in which individuals believe that persecution is undeserved) and bad me paranoia (in which persecution is believed to be deserved) [21] have been found to be differentially prevalent in early and later stages of psychosis $[22,23]$, suggestive of a qualitative difference occurring across the paranoia continuity. However, very few studies have investigated possible qualitative differences.

In the current study, participants with low, medium and high levels of trait paranoia were investigated to disentangle quantitative and/or qualitative differences in the effect of the social environment on fluctuations in momentary paranoia. Specifically, we investigated i) whether trait paranoia was associated with differences in time spent in different social contexts (e.g. being alone vs. being with others); ii) whether social features of the situation were predictive of momentary paranoia and whether this was different between the low, medium and high paranoid participants; iii) whether the effects on momentary paranoia were specific for social contextual effects or whether they could be replicated for another type of stressor (i.e. event stress).

\section{Methods}

\section{Sample}

In order to obtain a sample that ranged across the continuum of paranoia, the following individuals who differed in level of current paranoid symptomatology were included: i) patients diagnosed with a psychotic disorder who currently presented paranoid psychotic symptoms, defined as having a score of $>3$ on item P6 (suspiciousness) of the Positive and Negative Syndromes Scale (PANSS)[24]; ii) patients diagnosed with a psychotic disorder who currently had other positive psychotic symptoms, defined as having a score of $<4$ on the PANSS items P6 (suspiciousness) AND having a score of $>3$ on at least one of the PANSS items P1 (delusions), P3 (hallucinatory behaviour), P5 (grandiosity) and G9 (unusual thought content); iii) patients diagnosed with a psychotic disorder who reported remitted psychotic symptoms, defined as having a score of $<4$ on all the aforementioned PANSS items; iv) individuals with no diagnosis of psychotic disorder who presented a psychometric at risk mental state for paranoid psychosis (hereafter 'high schizotypy participants'), defined as scoring high ( $>90^{\text {th }}$ percentile) on the paranoid items of a questionnaire measuring psychosis-proneness (Community Assessment of Psychic Experiences, CAPE)[25]; and v) 'healthy' control participants defined in terms of scoring in the average range (between $45^{\text {th }}-55^{\text {th }}$ percentile) on all three symptom dimensions of the CAPE, and not scoring high ( $>90^{\text {th }}$ percentile) on the paranoid items [for a full description of this sample, see 26].

The study was approved by the local ethics committee, and all participants gave written informed consent. The inclusion criteria for all participants were age 18 to 65 years and sufficient command of the Dutch language to understand and fill out the questionnaires. Patients were recruited from inpatient 
and outpatient mental health facilities in the cities of Heerlen and Maastricht, the Netherlands. They were extensively screened in clinical interviews for psychiatric symptoms using the PANSS and the Life Chart [27]. Interview data and, when necessary, clinical record data were used to compose ICD-10 diagnoses using the OPCRIT computer program [28]. High schizotypy participants and healthy controls were recruited from an earlier longitudinal family study in the general population conducted in the city of Sittard, the Netherlands (Continuum of Mental Disorders Study, COMED)[for a full description of selection procedure, see 29].

\section{Instruments}

\section{Paranoia Scale (PS)}

The Paranoia Scale is a 20 -item questionnaire developed to measure subclinical levels of paranoid ideation [30]. It has been validated in a population of individuals diagnosed with schizophrenia [31]. All items are scored on a 5-point scale from not at all applicable to me (1) to extremely applicable to me (5). Good internal consistency (alpha $=0.84$ ) and stability $(r=0.70)$ of the PS have been established. Mean scores on the PS were conceptualized as measures of trait paranoia, with higher scores reflecting higher levels of trait paranoia (scores ranging from 20 to 100). For the current study, the PS showed excellent internal consistency (Cronbach's alpha $=0.92$ ). The PS score distribution was divided by its tertiles, creating tertile groups (= trait paranoia groups; LP = low paranoia, MP = medium paranoia, HP = high paranoia) (see Table 1 for composition).

\section{The Experience Sampling Method (ESM)}

Paranoid ideations and social context variables were assessed with ESM $[12,13]$. This is a random timesampling, self-assessment technique to assess mental state and context in normal daily life. Participants received a digital wristwatch and ESM assessment forms collated in a booklet for each day. Ten times a day on 6 consecutive days, the watch emitted a signal at unpredictable moments between 7.30 a.m. and 10.30 p.m. After every "beep", subjects were asked to fill out the ESM self-assessment forms previously handed to them, collecting reports of thoughts, current context (activity, persons present and location), appraisals of the current situation, mood and psychotic experiences. All self-assessments were rated on 7-point Likert scales. Open-ended questions were used to collect information on the social company at the time of each signal. Participants were instructed to complete their reports immediately after the beep, to minimize memory distortions, and to record the time at which they completed the form. Reports are assumed valid when subjects respond to the beep within 15 minutes. This was ascertained by comparing the actual beeping time with the reported time of completion of the reports. All reports completed more than 15 minutes after the signal were excluded from the analyses. Participants with less than 20 valid beeps were excluded from the analyses. Previous studies have demonstrated the feasibility, validity, and reliability of ESM in general and patient populations [13]. 


\section{ESM measures}

Social environment \& company. At each beep, participants had to indicate whether they were alone or in social company (alone: $0=$ yes and $1=$ no). When not alone, participants were asked to specify the kind of social company they were in (social company: 0 = being with familiar individuals (partner, children, siblings, other relatives and friends) and 1 = being with less-familiar individuals (colleagues, neighbors, health care professionals, other acquaintances and strangers)).

Momentary paranoia. In accordance with previous work [26], momentary paranoia was defined as the mean score of the ESM items I feel suspicious, I feel safe (reversed score), I feel that others dislike me, and I feel that others might hurt me. All items were rated on 7-point Likert scales (ranging from not at all to very) ( $\alpha=.82)$.

Perceived social threat. Since we sampled subjects across the range of paranoia, we also included a more subtle indicator of paranoia. In case participants were not alone at the time of the beep, they evaluated their social company. The mean score of the ESM items I like this company (reversed score),, In this company, I feel threatened, In this company, I feel accepted (reversed score), and I would rather be alone, rated on 7-point Likert scales (ranging from not at all to very), was conceptualized as a continuous variable of perceived social threat (Cronbach's $\alpha=.71$ ).

Event stress. In accordance with previous work, stress was conceptualized as the subjectively appraised stressfulness of distinctive events (event stress) [32]. Participants were asked to report the most important event that happened between the current and the previous beep. Subsequently, they evaluated its valence on a 7-point bipolar scale (ranging from $-3=$ very unpleasant, $0=$ neutral, to $3=$ very pleasant). The variable event stress was recoded so that higher values presented higher stress levels.

\section{Statistical Analyses}

Multilevel linear and logistic modeling techniques, which are ideally suited for analyses of clustered data [33], were used since ESM data have a hierarchical structure with repeated momentary measurements (level 1) per subject (level 2). Analyses were carried out with the XTREG module and the XTGEE module in STATA/MP version 10.0 [34]. Outcome variables included in the analyses were standardized by dividing the variables by the standard deviation of this variable over the group, yielding standardized effect sizes. All multilevel models were corrected for the a priori selected confounders sex and age.

Main effects and interactions were assessed by Wald test. The size of moderator effects was calculated by applying and testing the appropriate linear combinations using the STATA LINCOM command. Since we were interested in identifying quantitative versus qualitative differences across the range of paranoia, trait paranoia was included as a categorical variable in the analyses (1=low paranoia, 2=medium paranoia, 3=high paranoia). 


\section{i) Association between trait paranoia and the social environment}

Associations between trait paranoia group and the frequency of moments participants spend alone, with familiar individuals and with less-familiar individuals were investigated. Two multilevel logistic regression models were estimated with trait paranoia group as the independent variable and respectively alone and type of social company as the dependent variables.

\section{ii) Association between momentary paranoia and the social environment}

In order to examine the association between being alone and momentary paranoia, a multilevel linear regression analysis was estimated with momentary paranoia as the dependent variable and 'alone' and 'trait paranoia', as well as their interaction as independent variables. The interaction term (alone*trait paranoia group) was of major interest as the main hypothesis revolved around the question whether trait paranoia group moderated the association between being alone and momentary paranoia. In order to estimate effect sizes for each of the trait paranoia groups separately, linear combinations were calculated. Similar analyses were conducted with social company as the independent variable and momentary paranoia and perceived social threat respectively as the outcome variables.

Paranoia at the previous moment and perceived social threat at the previous moment respectively were separately added as covariates, since preceding levels of paranoia or social threat may influence the choice of company.

\section{iii) Association between momentary paranoia and subjectively appraised stress}

All multilevel linear regression models with momentary paranoia as the dependent variable and trait paranoia group as the predictor variable were repeated with event stress as predictor variable.

\section{Results}

\section{Sample and Descriptive Statistics}

Of the 183 participants in the study, 29 (15.8\%) were excluded from the analyses. Twenty-two participants terminated the study before the end of the 6-day sampling period, due to severity of psychotic symptoms ( $n=11)$, not being able to understand the instructions $(n=5)$, or lack of cooperation $(n=6)$. Although they finished the study, 3 individuals were excluded because of an insufficient number $(<20)$ of valid ESM observations, 1 individual because of missing data on the PS, 1 individual because of missing data on the PANSS, and 2 individuals because of missing data on both the PS and the PANSS. Of the 29 individuals who were excluded from the analyses, 24 were patients, 3 were high-schizotypy 
participants, and 2 were healthy controls. Drop-out was not associated with levels of event stress, momentary paranoia, momentary perceived social threat or trait paranoia. The final study sample therefore comprised 154 participants. These participants had each completed an average of 44 valid ESM reports $(S D=10)$. Additional information regarding number of valid reports and sociodemographic and clinical characteristics of the sample are depicted in Table 2 and Table 3.

\section{i) Association between trait paranoia and the social environment}

Trait paranoia group was not significantly associated with the amount of time participants spent alone $\left(\chi^{2}(2)=3.30, p=0.19\right)$ (see Figure 1a for percentages). Moreover, trait paranoia group was not associated with the relative amount participants spent with either familiar or less-familiar individuals $\left(\chi^{2}(2)=2.02\right.$, $p=0.36$ ) (see Figure $1 b$ for percentages).

\section{ii) Association between momentary paranoia and the social environment}

There was no significant main effect of being alone on momentary paranoia ( $\beta[S E]=.009[.02] ; p=.68$ ). Moreover, multilevel analyses revealed no significant interaction between trait paranoia group and being alone in the model of momentary paranoia $\left(\chi^{2}(2)=2.28, p=0.32\right)$. A marginal main effect was found for type of social company in the model of momentary paranoia ( $\beta[S E]=.07$ [.04]; $p=.052$ ), with more momentary paranoia in the company of less familiar individuals. In addition, level of trait paranoia moderated the effect of social company on momentary paranoia $\left(\chi^{2}(2)=10.02, p<0.01\right)$. In the low and medium paranoia group, more paranoia was reported when in the company of less-familiar people (Table 4). The high paranoia group reported no difference in paranoia when they were in less-familiar compared to more familiar company (Table 4).

For perceived social threat, a main effect was found for the type of social company, suggesting more perceived social threat in the company of less-familiar individuals $(\beta[S E]=.36[.04] ; p<.001)$. Again, a significant interaction was apparent between trait paranoia and social company $\left(\chi^{2}(2)=31.59, p<0.001\right)$. Low and medium paranoid subjects reported higher levels of perceived social threat when with lessfamiliar compared to familiar individuals, whereas no difference was found for the high paranoia group (Table 4). After controlling for paranoia or perceived social threat at the previous moment, all interaction effects remained significant.

iii) Association between momentary paranoia and subjectively appraised stress

Multilevel analyses revealed a significant interaction between paranoia group and event stress in the model of momentary paranoia $\left(\chi^{2}(2)=12.86, p<0.01\right)$. Higher levels of event stress predicted higher 
levels of momentary paranoia in all groups. However, the effect was largest in the high paranoia group $(\beta[S E]=.06[.01] ; p<.001)$, medium large in the second paranoia tertile $(\beta[S E]=.04[.01] ; p<.001)$ and smallest in the low paranoia group $(\beta[S E]=.03[.004] ; p<.001)$.

Slightly decreased but still significant effect sizes were found when controlling for paranoia at the previous moment.

\section{Sensitivity Analysis}

Additional analyses were performed to investigate whether inclusion of the participants, who were excluded due to an insufficient number of valid ESM observations, influenced the results. Apart from some small effect size alterations, all results remained unchanged.

\section{Discussion}

The present study investigated the effect of real-life social situations on moment-to-moment changes in paranoia. It was shown that social situations may be particularly relevant in the variability of low and mild levels of paranoia, whereas high levels of paranoia may become autonomous and independent of the social environment. However, general levels of subjective distress were important predictors of paranoid thinking at all levels of trait paranoia and indeed the most paranoid individuals showed the greatest reactivity to subjective stress.

\section{Trait paranoia and social context}

There were no differences between the paranoia groups in time spent alone versus time spent in company. Moreover, there were no differences between the groups in time spent in familiar company versus time spent in less-familiar company. This may seem counterintuitive, since, for example, the overall size of the social networks of psychotic patients is often smaller than in control groups [35]. Moreover, social avoidance as a result of safety behaviors has been related to trait paranoia [36, 37]. Likewise, social withdrawal is a common phenomenon emerging prior to manifestation of psychosis [38]. However, our results are in line with the results of another ESM study that reported no effect of psychosis-proneness on time spent alone or with familiar individuals [39]. Our results thus suggest that individuals with high levels of paranoia are not necessarily alone more often during everyday life, even though their social networks may be smaller. Demographic characteristics of the three groups can possibly explain these results. Although few participants of the high paranoia group lived together with a partner or family, about $57 \%$ of them were living on a psychiatric ward or in supported accommodation with other patients, probably resulting in more social contact. 


\section{Momentary paranoia and the social environment}

The current study found a different association between the type of social company and momentary fluctuations in paranoid ideation dependent on trait paranoia. Type of social company most prominently affected momentary paranoia in the medium paranoia group, who experienced increases in momentary paranoia and perceived social threat when in the company of less-familiar people. This result concurs with earlier findings. In Verdoux's study (2003), a non-clinical population with a high level of vulnerability for psychosis experienced an increase in psychotic experiences when in non-familiar compared to familiar social company [16]. Moreover, an effect of social company on paranoia for high-schizotypy groups in particular has been confirmed repeatedly by virtual reality studies [3].

Interestingly, these effects were absent at high levels of trait paranoia. This seems in contrast with the results of the study by Myin-Germeys et al. (2001), who found a decreased risk of experiencing delusions when patients were in familiar social company [11]. A possible explanation for this difference might be the composition of samples. Whereas in the current study participants were divided on basis of their level of trait paranoia, the patient sample in the study by Myin-Germeys et al. (2001) consisted mostly of stable, chronic patients, possibly with medium rather than high levels of trait paranoia.

Interestingly, the qualitative differences we found between paranoia groups seem to be specific for the association between paranoia and social company, which is a relatively "objective" contextual measure. For event stress, which reflects the subjective appraisal of an event as stressful, we found quantitative differences of paranoid reactivity between the groups. The low paranoia group experienced the least paranoia in response to event stress, while the high paranoia group experienced the most, suggesting a dose-response effect on paranoid thinking.

These findings suggest that for highly paranoid individuals, subjective experiences of stress have become decoupled from social context.

\section{Underlying Mechanisms}

The current findings suggest that superimposed on the continuity of psychosis, which has been supported by a meta-analysis of data on the distribution of psychotic symptoms [18], there may be qualitative differences between those with severe psychotic experiences and those whose experiences are less severe. Our observations are unlikely to reflect a ceiling effect for paranoid thinking because even those participants who were high on trait paranoia showed fluctuations in response to subjectively appraised stress.

The results obtained from the low and medium paranoia groups suggest that sensitivity to social context is a normal, perhaps adaptive, process; when exposed to unfamiliar people, one may be inclined to be wary and suspicious of their intentions but when encountering someone with whom one has a long 
history of positive interactions, wariness may not be warranted. The failure to take into account this social contextual information may be a core feature of severe paranoid delusions.

It is possible that this finding helps to explain other discontinuities which have been observed across the paranoid spectrum. Attributional style (reasoning about causes of events) [40] has been found to differ across the range of paranoia. Some studies have reported that paranoid patients make excessive external attributions for negative events [e.g. 41, 42], but this style seems to be absent in less severely paranoid non-clinical samples [41, 43,44]. Furthermore, acutely ill paranoid patients predominantly have "poor me" paranoid beliefs in which they believe that they do not deserve to be persecuted [22, 45], but less severely paranoid non-patients typically report "bad me" beliefs, in which they believe that they do deserve to be persecuted [46]. The relationship between these discontinuities is as yet poorly understood. One possibility is that, in the absence of the ability to adjust suspiciousness and perception of threat according to context, paranoid beliefs will escalate leading to the generalized assumption that misfortunes are always caused by the intentional action of others (externalizing attributional style) together with the belief that one is being undeservedly persecuted (poor me paranoia).

Recent evidence suggests that the striatal dopamine system may play a role in threat-anticipation that parallels its well-documented role in reward learning [47]. Hence, the finding that at a high level of paranoia, social threat anticipation and paranoid thinking are independent of social context may be consistent with the hypothesis, supported by some recent evidence [7], that acute psychosis is associated with a hypersensitized dopamine system that operates independently of context [48]. Evidence from recent $\mathrm{fMRI}$ research is also in line with the present findings, suggesting that paranoid individuals with schizophrenia in contrast to non-paranoid individuals with schizophrenia and a control group exhibit impaired modulation of neural activity when processing social stimuli [49].

\section{Methodological issues}

Measurements of momentary paranoia, perceived social threat, event stress and the social environment were based on subjective reports. Although it is sometimes assumed that subjective reports can be considered less reliable than objective measures, they can be valid, whereas the validity of objective approaches should not be taken for granted [50]. Second, the current study used a daily life assessment technique in which participants had to comply with a paper-and-pencil diary protocol without the researcher being present, making it impossible to determine directly whether patients filled in the booklets after the beeps or whether they were backfilling their diaries. Therefore, some authors have cast doubt on the reliability and subject compliance in paper-and-pencil ESM studies, favoring the use of electronic devices [51]. However, in a comparative study, Green et al. concluded that both methods yielded similar results [52]. With a paper and pencil approach, participants are instructed to write down the time when they filled out the assessment and only those assessments filled out within a 15 minutes time frame of the beep are considered valid. If participants want to backfill the diary, they at 
least have to carry the watch and note all the times, since beeps are presented randomly over the day. A recent study of our group using a signal-contingent random time sampling procedure with multiple observations per day - such as the protocol used in the current study -found evidence underscoring acceptable compliance rates and thus the validity of the paper-and-pencil random time self-report data in the current study [53]. The research sample was specifically selected for paranoid symptoms. Therefore, conclusions cannot be generalized to delusions in general.

\section{Conclusions}

The data suggest that important differences exist across the range of trait paranoia in the effect of social context on paranoid thinking. The development of momentary paranoia appears to be contextdependent in individuals with medium or at-risk levels of trait paranoia, probably reflecting adaptive processes. However, at high levels of trait paranoia, momentary paranoia and momentary perceived social threat seem to become autonomous and independent of the social reality.

Further examination of these effects may have implications for the clinical management of paranoid patients. Cognitive behavioural therapy for paranoid symptoms usually involves encouraging patients to reflect on the social context of their symptoms and question their interpretations of events [54]. If paranoid thoughts are autonomous of social context patients may have difficulty doing this. It seems possible that approaches which encourage detached acceptance of unpleasant thoughts, for example acceptance and commitment therapy, will be more effective. A small randomized controlled trail has reported positive effects of acceptance commitment therapy for patients recovering from acute psychosis [55].

Funding This work was supported by a 2006 National Alliance for Research on Schizophrenia and Depression (NARSAD) Young Investigator Award and by the Dutch Medical Research Council, VIDI grant [grant number 917.76.341] to I.M.-G.

Acknowledgments The authors wish to thank Truda Driesen and Frieda van Goethem for data administration. 
Table 1. Composition of the paranoia tertile groups

\begin{tabular}{l|lll}
\hline Sample $N=154$ & $\begin{array}{l}\text { Tertile group 1 } \\
\text { Low paranoia } \\
(n=55)\end{array}$ & $\begin{array}{l}\text { Tertile group 2 } \\
\text { Medium paranoia } \\
(n=48)\end{array}$ & $\begin{array}{l}\text { Tertile group } 3 \\
\text { High paranoia } \\
(n=51)\end{array}$ \\
\hline Healthy controls & $84 \%(n=31)$ & $16 \%(n=6)$ & $0 \%(n=0)$ \\
High schizotypy participants & $36 \%(n=13)$ & $56 \%(n=20)$ & $8 \%(n=3)$ \\
Remitted patients & $27 \%(n=4)$ & $33 \%(n=5)$ & $40 \%(n=6)$ \\
Low paranoid patients & $18 \%(n=6)$ & $29 \%(n=10)$ & $53 \%(n=18)$ \\
High paranoid patients & $3 \%(n=1)$ & $23 \%(n=7)$ & $74 \%(n=23)$ \\
\hline
\end{tabular}

Note. Due to rounding, percentages may not add exactly to $100 \%$. 
Table 2. Subsample characteristics

\begin{tabular}{|c|c|c|c|c|c|c|}
\hline & $\begin{array}{l}\text { Healthy } \\
\text { controls } \\
(n=38)\end{array}$ & $\begin{array}{c}\text { High } \\
\text { schizotypy } \\
\text { participants } \\
(n=36)\end{array}$ & $\begin{array}{l}\text { Remitted } \\
\text { patients } \\
\quad(n=15)\end{array}$ & $\begin{array}{l}\text { Current } \\
\text { non- } \\
\text { paranoid } \\
\text { patients } \\
(n=34)\end{array}$ & $\begin{array}{l}\text { Current } \\
\text { paranoid } \\
\text { patients } \\
(n=30)\end{array}$ & \\
\hline Age, years: mean (SD) & $48(9.3)$ & $47(10.5)$ & $33(12.3)$ & $36(11.6)$ & $38(10.6)$ & $\mathrm{F}=12.01 * * *$ \\
\hline Gender (male:female) & $14: 24$ & $12: 24$ & $14: 1$ & $26: 8$ & $26: 4$ & $\chi^{2}(4)=36.4^{* * *}$ \\
\hline Education, No. (\%)* & & & & & & $\chi^{2}(8)=37.9 * * *$ \\
\hline Elementary school & $1(2.7)$ & $2(5.6)$ & $1(6.7)$ & $6(17.7)$ & $3(10)$ & \\
\hline Secondary school & $15(39.5)$ & $21(58.3)$ & $14(93.3)$ & $22(64.7)$ & $25(83.3)$ & \\
\hline Higher education & $22(57.9)$ & $13(36.1)$ & $0(0)$ & $6(17.7)$ & $2(6.7)$ & \\
\hline Marital status, No. (\%) & & & & & & $\chi^{2}(12)=95.9 * * *$ \\
\hline Married or living together & $33(86.8)$ & $27(75)$ & $1(6.7)$ & $2(5.9)$ & $3(10)$ & \\
\hline Divorced & $4(10.5)$ & $4(11.1)$ & $1(6.7)$ & $5(14.7)$ & $6(20)$ & \\
\hline Widowed & - & - & - & $1(2.9)$ & - & \\
\hline Never married/single & $1(2.6)$ & 5 (13.9) & $13(86.7)$ & $26(76.5)$ & $21(70)$ & \\
\hline Work situation, No. (\%)* & & & & & & $\chi^{2}(12)=96.1^{* * *}$ \\
\hline $\begin{array}{l}\text { Working/significant housework/ } \\
\text { studying }\end{array}$ & $34(89.5)$ & $23(63.9)$ & $2(14.3)$ & $2(6.3)$ & $1(3.3)$ & \\
\hline Protected work & $1(2.6)$ & $7(19.4)$ & $3(21.4)$ & $4(12.5)$ & $5(16.7)$ & \\
\hline Incapable of work & $2(5.3)$ & $5(13.9)$ & $9(64.3)$ & $26(81.3)$ & $24(80)$ & \\
\hline Unemployed & - & - & - & - & - & \\
\hline Retired & $1(2.6)$ & $1(2.8)$ & - & - & - & \\
\hline Living situation, No. (\%)* & & & & & & $\chi^{2}(16)=112^{* * *}$ \\
\hline Alone & $3(7.9)$ & $4(11.1)$ & $1(6.7)$ & $6(17.7)$ & $6(20)$ & \\
\hline With partner/family/childr & $35(92.1)$ & $28(77.8)$ & $1(6.7)$ & $3(8.8)$ & $3(10)$ & \\
\hline With parents/relatives & - & $2(5.6)$ & $3(20)$ & $2(5.9)$ & $1(3.3)$ & \\
\hline $\begin{array}{l}\text { Ward or supported } \\
\text { accommodation } \\
\text { Other }\end{array}$ & - & $2(5.6)$ & $\begin{array}{l}9(60) \\
1(6.7)\end{array}$ & $\begin{array}{l}21(61.8) \\
2(5.9)\end{array}$ & $20(66.7)$ & \\
\hline OPCRIT lifetime ICD-10 diagnosis, No.(\%)* & & & & & & $\chi^{2}(12)=161.9 * * *$ \\
\hline Schizophrenia/psychotic disorder & - & - & $15(100)$ & $28(82.4)$ & $28(93.3)$ & \\
\hline Schizoaffective disorder & - & - & - & $6(17.7)$ & $2(6.7)$ & \\
\hline Mild/Moderate depression & $6(15.8)$ & $4(11.1)$ & - & - & - & \\
\hline No diagnosis & $32(84.2)$ & $32(88.9)$ & - & - & - & \\
\hline PS score ${ }^{a}$ & $32.8(7.3)$ & $41.6(9.7)$ & $46.3(13.7)$ & $52.7(13.7)$ & $64.1(16.5)$ & $\mathrm{F}=2.85^{* * *}$ \\
\hline PANSS score ${ }^{b}$ & $31.7(2.9)$ & $34.1(4.2)$ & $41.7(8)$ & $57.4(9.1)$ & $65.2(17.6)$ & $\mathrm{F}=77.3 * * *$ \\
\hline No. of valid reports, mean (SD) & $48.75 .8)$ & $49.4(6.6)$ & $42.2(8.9)$ & $38.03(9.6)$ & $36.6(10)$ & $\mathrm{F}=17.9 * * *$ \\
\hline Momentary paranoia, mean (SD) & $1.3(0.3)$ & $1.6(0.6)$ & $1.7(0.7)$ & $2.1(0.8)$ & $3.2(1.5)$ & $\mathrm{F}=26.2 * * *$ \\
\hline Perceived social threat, mean (SD) & $1.5(0.3)$ & $1.6(0.5)$ & $1.9(0.7)$ & $2.4(0.8)$ & $2.5(1)$ & $\mathrm{F}=15.6 * * *$ \\
\hline Event stress, mean (SD) & $-1.6(0.6)$ & $-1.5(0.6)$ & $-1.7(0.6)$ & $-1.3(1)$ & $-1.3(1)$ & $\mathrm{F}=1.2$ \\
\hline Age first psychotic episode, mean (SD) ${ }^{c}$ & - & - & $22.1(6.3)$ & $23.1(7.3)$ & $22.7(8.2)$ & $F=0.08$ \\
\hline
\end{tabular}


Table 3. ESM variables per paranoia tertile

\begin{tabular}{l|llll}
\hline & $\begin{array}{l}\text { Low } \\
\text { paranoia } \\
(\mathrm{n}=55)\end{array}$ & $\begin{array}{l}\text { Medium } \\
\text { paranoia } \\
(\mathrm{n}=48)\end{array}$ & High paranoia & $\mathrm{F}(2,151)$ \\
$(\mathrm{n}=51)$ & \\
\hline Number of valid reports, Mean (SD) & $46.7(8.95)$ & $44.5(9.02)$ & $39.2(9.96)$ & $8.86^{* * *}$ \\
Momentary paranoia, Mean (SD) & $1.4(0.47)$ & $1.8(0.09)$ & $2.8(1.2)$ & $34.16^{* * *}$ \\
Perceived social threat, Mean (SD) & $1.5(0.48)$ & $1.8(0.59)$ & $2.6(0.92)$ & $33.09^{* * *}$ \\
Event stress, Mean (SD) & $-1.6(0.7)$ & $-1.4(0.7)$ & $-1.3(1.0)$ & 2.55 \\
\hline
\end{tabular}

Note. Separate means were calculated for each participant and subsequently aggregated to obtain group means. ${ }^{* * *} \mathrm{p}<0.001$

Table 4. Effect of social environment on momentary paranoia assessed by paranoia group

\begin{tabular}{|c|c|c|c|c|}
\hline & & Low paranoia & Medium paranoia & High paranoia \\
\hline Paranoia & $\begin{array}{l}\text { Type of social } \\
\text { company } \\
\beta^{\text {a }}(95 \% \mathrm{Cl} ; \mathrm{p})\end{array}$ & $\begin{array}{l}\mathbf{0 . 0 7} \\
(-0.001,0.13 ; p=0.05)\end{array}$ & $\begin{array}{l}\mathbf{0 . 1 1} \\
(0.03,0.2 ; p<0.01)\end{array}$ & $\begin{array}{l}-0.07 \\
(-0.16,0.02 ; p=0.10)\end{array}$ \\
\hline $\begin{array}{l}\text { Perceived } \\
\text { social } \\
\text { threat }\end{array}$ & $\begin{array}{l}\text { Type of social } \\
\text { company } \\
\beta^{b}(95 \% \mathrm{Cl} ; p)\end{array}$ & $\begin{array}{l}0.36 \\
(0.27,0.45 ; p<0.001)\end{array}$ & $\begin{array}{l}\mathbf{0 . 4 9} \\
(0.39,0.60 ; p<0.001)\end{array}$ & $\begin{array}{l}0.07 \\
(-0.05,0.18 ; p=0.26)\end{array}$ \\
\hline
\end{tabular}

\footnotetext{
${ }^{a}$ Regression coefficient indicates change in ESM paranoia associated with being in familiar company versus being in non-familiar company, analyses adjusted for age and sex.

${ }^{\mathrm{b}}$ Regression coefficient indicates change in ESM perceived social threat associated with being in familiar company versus being in non-familiar company, analyses adjusted for age and sex.
} 
Figure 1. Percentage of time spent alone versus not alone and percentage of time spent in familiar company versus non-familiar comapny by group

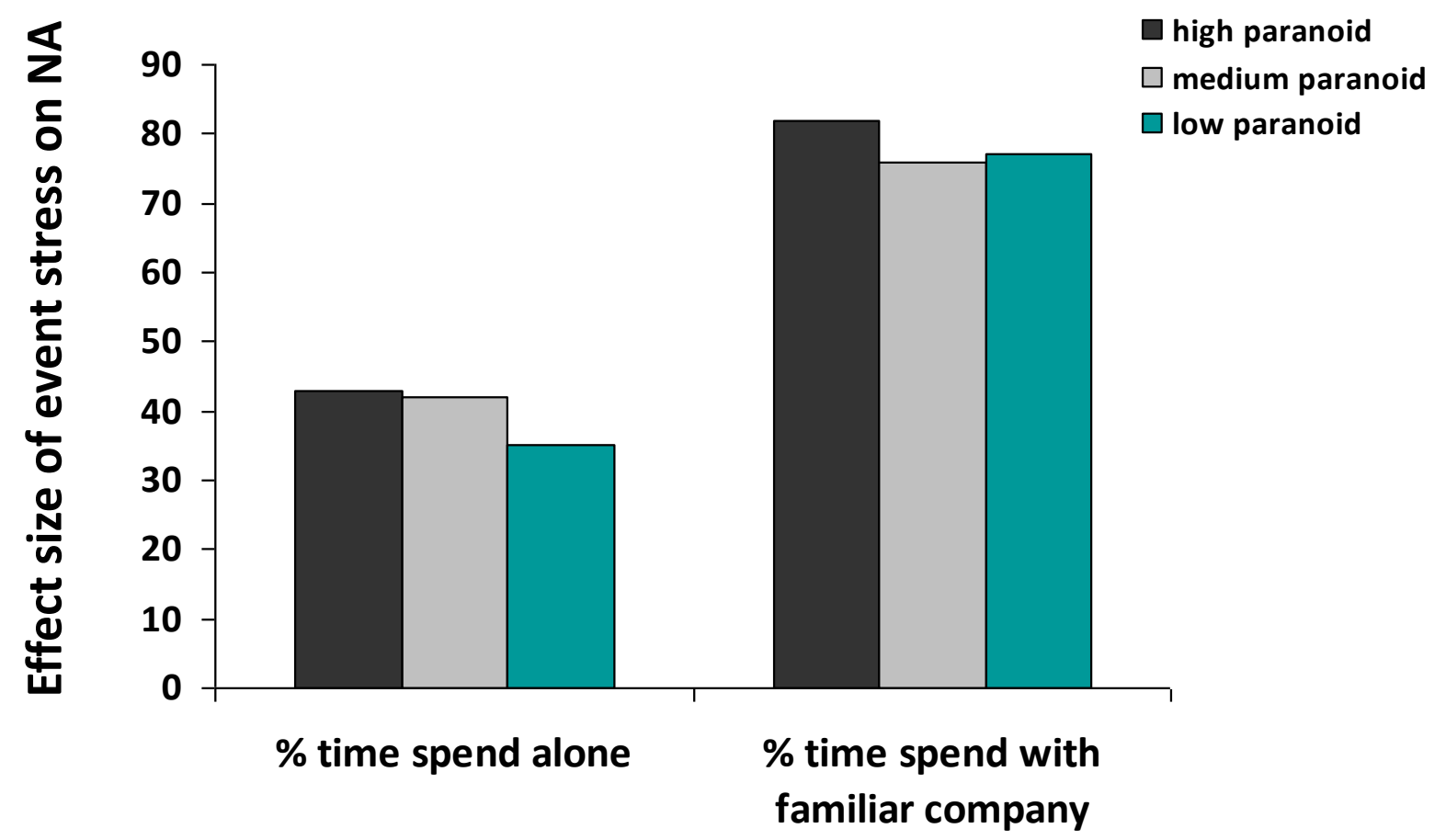




\section{References}

1. Garety PA and Hemsley DR (1987): Characteristics of delusional experience. Eur Arch Psychiatry Neurol Sci 236:2948.

2. Jorgensen P (1994): Course and outcome in delusional disorders. Psychopathology 27:79-88.

3. Freeman D (2008): Studying and treating schizophrenia using virtual reality: a new paradigm. Schizophr Bull 34:60510.

4. Bentall RP, Corcoran R, Howard R, Blackwood N, and Kinderman P (2001): Persecutory delusions: a review and theoretical integration. Clin Psychol Rev 21:1143-92.

5. Freeman D and Garety PA (2000): Comments on the content of persecutory delusions: does the definition need clarification? The British journal of clinical psychology / the British Psychological Society 39 ( Pt 4):407-14.

6. Jensen J and Kapur S (2009): Salience and psychosis: moving from theory to practise. Psychol Med 39:197-8.

7. Roiser JP, Stephan KE, den Ouden HE, Barnes TR, Friston KJ, and Joyce EM (2009): Do patients with schizophrenia exhibit aberrant salience? Psychol Med 39:199-209.

8. Ellett L, Freeman D, and Garety PA (2008): The psychological effect of an urban environment on individuals with persecutory delusions: The Camberwell walk study. Schizophr Res.

9. Freeman D, Slater M, Bebbington PE, Garety PA, Kuipers E, Fowler D, et al. (2003): Can virtual reality be used to investigate persecutory ideation? J Nerv Ment Dis 191:509-14.

10. Freeman D, Garety PA, Bebbington P, Slater M, Kuipers E, Fowler D, et al. (2005): The psychology of persecutory ideation II: a virtual reality experimental study. J Nerv Ment Dis 193:309-15.

11. Myin-Germeys I, Nicolson NA, and Delespaul PA (2001): The context of delusional experiences in the daily life of patients with schizophrenia. Psychol Med 31:489-98.

12. Csikszentmihalyi M and Larson R (1987): Validity and reliability of the Experience-Sampling Method. J Nerv Ment Dis 175:526-36.

13. Myin-Germeys I, Oorschot M, Collip D, Lataster J, Delespaul P, and van Os J (2009): Experience sampling research in psychopathology: opening the black box of daily life. Psychol Med:1-15.

14. Oorschot M, Kwapil T, Delespaul P, and Myin-Germeys I (2009): Momentary assessment research in psychosis. Psychol Assess 21:498-505.

15. Delespaul P, deVries M, and van Os J (2002): Determinants of occurrence and recovery from hallucinations in daily life. Soc Psychiatry Psychiatr Epidemiol 37:97-104.

16. Verdoux H, Husky M, Tournier M, Sorbara F, and Swendsen JD (2003): Social environments and daily life occurrence of psychotic symptoms--an experience sampling test in a non-clinical population. Soc Psychiatry Psychiatr Epidemiol 38:654-61.

17. Freeman D (2007): Suspicious minds: the psychology of persecutory delusions. Clin Psychol Rev 27:425-57.

18. van Os J, Linscott R, Myin-Germeys I, Delespaul P, and Krabbendam L (2009): A systematic review and meta-analysis of the psychosis continuum: evidence for a psychosis proneness-persistence-impairment model of psychotic disorder. Psychol Med 39:179-95.

19. Kapur S, Mizrahi R, and Li M (2005): From dopamine to salience to psychosis--linking biology, pharmacology and phenomenology of psychosis. Schizophr Res 79:59-68.

20. Moutoussis M, Williams J, Dayan P, and Bentall RP (2007): Persecutory delusions and the conditioned avoidance paradigm: towards an integration of the psychology and biology of paranoia. Cognit Neuropsychiatry 12:495-510.

21. Trower P and Chadwick P (1995): Pathways to defence of the self: A theory of two types of paranoia. Clinical Psychology: Science and Practice 2:263-277.

22. Fornells-Ambrojo M and Garety PA (2005): Bad me paranoia in early psychosis: a relatively rare phenomenon. Br J 
Clin Psychol 44:521-8.

23. Chadwick PD, Trower P, Juusti-Butler TM, and Maguire N (2005): Phenomenological evidence for two types of paranoia. Psychopathology 38:327-33.

24. Kay SR, Fiszbein A, and Opler LA (1987): The positive and negative syndrome scale (PANSS) for schizophrenia. Schizophr Bull 13:261-76.

25. Konings M, Bak M, Hanssen M, van Os J, and Krabbendam L (2006): Validity and reliability of the CAPE: a self-report instrument for the measurement of psychotic experiences in the general population. Acta Psychiatr Scand 114:5561.

26. Thewissen V, Bentall RP, Lecomte T, van Os J, and Myin-Germeys I (2008): Fluctuations in self-esteem and paranoia in the context of daily life. J Abnorm Psychol 117:143-53.

27. Susser E, Finnerty M, Mojtabai R, Yale S, Conover S, Goetz R, et al. (2000): Reliability of the life chart schedule for assessment of the long-term course of schizophrenia. Schizophr Res 42:67-77.

28. McGuffin P, Farmer A, and Harvey I (1991): A polydiagnostic application of operational criteria in studies of psychotic illness. Development and reliability of the OPCRIT system. Arch Gen Psychiatry 48:764-70.

29. Hanssen M, Peeters F, Krabbendam L, Radstake S, Verdoux H, and van Os J (2003): How psychotic are individuals with non-psychotic disorders? Soc Psychiatry Psychiatr Epidemiol 38:149-54.

30. Fenigstein A and Vanable PA (1992): Paranoia and self-consciousness. J Pers Soc Psychol 62:129-138.

31. Smári J, Stefánsson S, and Thorgilsson H (1994): Paranoia, self-consciousness, and social cognition in schizophrenics. Cognit Ther Res 18:387-399.

32. Myin-Germeys I and van Os J (2007): Stress-reactivity in psychosis: evidence for an affective pathway to psychosis. Clin Psychol Rev 27:409-24.

33. Schwartz JE and Stone AA (1998): Strategies for analyzing ecological momentary assessment data. Health Psychol $17: 6-16$.

34. StataCorp, (2007): Stata Statistical Software, Release 10.0. College Station, TX: Stata Corporation.

35. Macdonald EM, Hayes RL, and Baglioni AJ, Jr. (2000): The quantity and quality of the social networks of young people with early psychosis compared with closely matched controls. Schizophr Res 46:25-30.

36. Freeman D, Garety PA, and Kuipers E (2001): Persecutory delusions: developing the understanding of belief maintenance and emotional distress. Psychol Med 31:1293-306.

37. Freeman D, Garety PA, Kuipers E, Fowler D, Bebbington PE, and Dunn G (2007): Acting on persecutory delusions: the importance of safety seeking. Behav Res Ther 45:89-99.

38. Hoffman RE (2007): A social deafferentation hypothesis for induction of active schizophrenia. Schizophr Bull 33:1066-70.

39. Husky MM, Grondin OS, and Swendsen JD (2004): The relation between social behavior and negative affect in psychosis-prone individuals: an experience sampling investigation. Eur Psychiatry 19:1-7.

40. Kinderman P and Bentall RP (1996): Self-discrepancies and persecutory delusions: evidence for a model of paranoid ideation. J Abnorm Psychol 105:106-13.

41. Janssen I, Versmissen D, Campo JA, Myin-Germeys I, van Os J, and Krabbendam L (2006): Attribution style and psychosis: evidence for an externalizing bias in patients but not in individuals at high risk. Psychol Med 36:771-8.

42. Kaney S and Bentall RP (1989): Persecutory delusions and attributional style. Br J Med Psychol 62 (Pt 2):191-8.

43. Martin JA and Penn DL (2001): Social cognition and subclinical paranoid ideation. Br J Clin Psychol 40:261-5.

44. Combs DR and Penn DL (2004): The role of subclinical paranoia on social perception and behavior. Schizophr Res 69:93-104.

45.

Bentall RP, Rowse G, Kinderman P, Blackwood N, Howard R, Moore R, et al. (2008): Paranoid delusions in 
schizophrenia spectrum disorders and depression: the transdiagnostic role of expectations of negative events and negative self-esteem. J Nerv Ment Dis 196:375-83.

46. Melo S, Corcoran R, Shryane N, and Bentall RP (2009): The persecution and deservedness scale. Psychol Psychother 82:247-60.

47. Moutoussis M, Bentall RP, Williams J, and Dayan P (2008): A temporal difference account of avoidance learning. Network 19:137-60.

48. Laruelle M and Abi-Dargham A (1999): Dopamine as the wind of the psychotic fire: new evidence from brain imaging studies. J Psychopharmacol (Oxf) 13:358-71.

49. Pinkham AE, Hopfinger JB, Pelphrey KA, Piven J, and Penn DL (2008): Neural bases for impaired social cognition in schizophrenia and autism spectrum disorders. Schizophr Res 99:164-75.

50. Strauss JS (1994): The person with schizophrenia as a person. II: Approaches to the subjective and complex. British Journal of Psychiatry (Suppl):103-7.

51. Stone AA, Shiffman S, Schwartz JE, Broderick JE, and Hufford MR (2003): Patient compliance with paper and electronic diaries. Control Clin Trials 24:182-99.

52. Green AS, Rafaeli E, Bolger N, Shrout PE, and Reis HT (2006): Paper or plastic? Data equivalence in paper and electronic diaries. Psychol Methods 11:87-105.

53. Jacobs N, Nicolson NA, Derom C, Delespaul P, van Os J, and Myin-Germeys I (2005): Electronic monitoring of salivary cortisol sampling compliance in daily life. Life Sciences 76:2431-43.

54. Morrison AP, Renton J, Dunn H, Williams S, and Bentall R (2003): Cognitive therapy for psychosis: A formulationbased approach. 2003, Hove: Brunner-Routledge.

55. Bach P and Hayes SC (2002): The use of acceptance and commitment therapy to prevent the rehospitalization of psychotic patients: a randomized controlled trial. J Consult Clin Psychol 70:1129-39. 
${ }^{1}$ From the Department of Psychiatry and Neuropsychology, South Limburg Mental Health Research and Teaching Network, EURON, Maastricht University Medical Centre, Maastricht, The Netherlands

2 King's College London, King's Health Partners, Department of Psychosis Studies, Institute of Psychiatry, London, UK

* contributed equally 


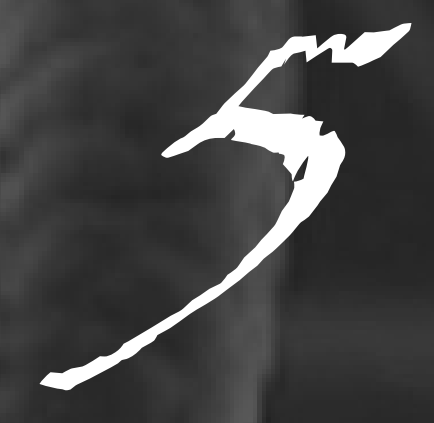

\section{Daily Cortisol, Stress Reactivity and Psychotic Experiences in Individuals at Above Average Genetic Risk for Psychosis}

Dina Collip ${ }^{1}$, Nancy A. Nicolson ${ }^{1 *}$, Mariëlle Lardinois ${ }^{1 *}$, Tineke Lataster ${ }^{1}$, Jim van Os $^{1,2} \&$ Inez Myin-Germeys ${ }^{1}$ for GROUP* 


\section{Abstract}

\section{Background}

Hypothalamic-pituitary-adrenocortical (HPA) axis abnormalities have been found in patients with a psychotic disorder and first-degree relatives of patients with a psychotic disorder react with subtle increases in non-clinical psychotic experiences and negative emotions in the face of everyday stress. The current study investigated whether HPA axis functioning is altered in individuals at above average genetic risk for psychotic disorder, examining diurnal cortisol profiles, cortisol reactivity to daily stressors and the association between HPA axis activity and subclinical psychotic experiences.

\section{Method}

Participants included siblings of patients with a psychotic disorder $(n=60)$ and a healthy comparison group ( $n=63$ ). The Experience Sampling Method (ESM; a structured diary technique) was employed to assess stress, psychotic experiences, negative affect and salivary cortisol repeatedly in the flow of daily life.

\section{Results}

Multilevel analyses revealed higher diurnal cortisol level and heightened cortisol reactivity to negative daily events in siblings compared to controls. Diurnal cortisol slope did not differ between the two groups, but momentary increases in psychotic experiences and negative affect were associated with increased cortisol in the sibling group.

\section{Conclusions}

Findings support altered HPA axis activity in individuals at above average genetic risk for psychotic disorder, as evidenced by higher diurnal cortisol levels and increased cortisol reactivity to daily stress. Results also suggest a dynamic association between cortisol secretion and the intensity of psychoticlike experiences and negative emotions in daily life, although the direction of this association remains to be elucidated.

Key words: psychosis/cortisol/HPA axis/momentary assessment/siblings/daily stress/negative affect 


\section{Introduction}

Minor stresses in the flow of daily life have repeatedly been associated with increases in psychotic symptoms and negative emotions in individuals with a psychotic disorder [1]. However, the biological substrate underlying this phenomenon remains unknown, and vulnerability markers need to be identified. Results of experimental studies suggest that increased psychotic reactivity to stress may reflect increased dopamine reactivity $[2,3]$. In addition, several lines of evidence indicate that dysregulation of the hypothalamic-pituitary-adrenocortical (HPA) axis may play a role in the relationship between stress and psychotic experiences $[4,5]$. In individuals with a psychotic disorder, abnormalities have been found at various levels of the HPA axis, including functional and structural changes in the hypothalamus [6], the pituitary [7], and the hippocampus [8]. Similarly, cortisol levels and reactivity to stress may be disturbed [9-17]. Nevertheless, it remains uncertain whether HPA axis abnormalities are causally related to the increased emotional and psychotic stress reactivity observed in patients with a psychotic disorder.

One of the major obstacles to research in this area is that patients with a psychotic disorder usually receive antipsychotic medication, which can affect cortisol levels and brain structures [7, 18, 19]; thus, studies in patient samples cannot yield firm conclusions about HPA axis dysregulation as biological substrate or vulnerability marker. Studies in patients with a first episode of psychotic illness are informative in that they are not confounded by long-term medication or illness effects; however, they cannot establish the degree to which increased HPA axis activity constitutes a precursor of psychosis, as reflected in an increased vulnerability to stress, as opposed to being the result of the psychosis and related stress. Therefore, studies have been conducted in persons who are prone to psychosis but have not developed a psychotic disorder. Schizotypal adolescents, for example, were found to have higher cortisol levels than controls [20], and heightened cortisol secretion in another sample of schizotypal adolescents was associated with schizotypal symptomatology at 2-year follow-up [21]. The potential predictive value of HPA axis abnormalities is further supported by findings in a sample of young people at ultra high risk (UHR) of psychotic disorder; months before onset, those who later developed a psychotic disorder had larger pituitary volumes than those who did not develop a disorder [22]. Walker et al. (2010) recently reported that higher cortisol levels predicted higher risk of conversion to psychotic disorder in an at-risk sample studied longitudinally. Together these findings add support to the hypothesis that heightened stress reactivity may be one of the risk factors underlying both HPA axis abnormalities and vulnerability to psychotic disorder.

Healthy siblings of individuals with a psychotic disorder are another important research population: they share not only genetic vulnerability for psychosis with their affected siblings, but also many sociodemographic, parental and developmental characteristics. First-degree relatives show increases in the intensity of subtle psychotic experiences and negative emotions in reaction to daily hassles [1, 23]. Moreover, studies have reported structural HPA axis abnormalities, such as increased pituitary [24] 
and hypothalamic volumes [25], in relatives of patients with schizophrenia. However, sibling studies have not yet examined cortisol secretory patterns or the possible association of cortisol with increased emotional and psychotic reactivity to stress in daily life.

The current study therefore assessed cortisol in a sample of siblings of patients with psychotic disorder and matched controls. We used the Experience Sampling Method, a structured diary technique, to assess daily hassles, psychotic experiences and negative emotions at frequent intervals over the course of several days, with concurrent sampling of salivary cortisol. In studies conducted in the natural environment, cortisol is generally characterized in terms of overall levels, diurnal patterns, and reactivity to acute stressors [26]. Cortisol has a pronounced circadian rhythm, with a sharp increase in the first hour after awakening and a gradual decrease over the rest of the day. Acute stressors can induce short-term peaks, with a half-life of approximately an hour. The design of the current study allowed investigation of several of these aspects of cortisol secretion. The aims were threefold: (i) to examine differences in overall cortisol levels and diurnal slope between siblings and controls, (ii) to investigate differences between siblings and controls in cortisol reactivity in response to naturally occurring stressors, and (iii) to determine the association between momentary psychotic experiences or negative emotions and momentary cortisol in siblings compared to controls. In case of significant group differences, we performed additional analyses to explore alternative (non-genetic) explanations of any difference in cortisol patterns (including effects of depression, childhood trauma, current stress and distress levels and degree of psychometric psychosis liability).

\section{Methods}

\section{Participants}

The sample consisted of 71 healthy siblings (68 full siblings and 3 half-siblings) of patients diagnosed with a non-affective psychotic disorder and 66 control subjects. The siblings were recruited through their ill relatives or family support organizations as part of a larger study [27]. Controls were selected through random mailings to addresses in the residential areas of patients and siblings. Written informed consent, conforming to local ethics committee guidelines, was obtained from all subjects. The Comprehensive Assessment of Symptoms and History (CASH) [28] was completed to assess symptom history over the past six months and lifetime, yielding DSM-IV diagnoses [29]. Inclusion criteria were: (i) age 16 to 55 years, and (ii) sufficient command of the Dutch language. Exclusion criteria were: (i) use of steroid medication; (ii) current axis 1 disorder; (iii) lifetime history of psychotic disorder; and, for the controls, (iv) family history of psychotic disorder as assessed by the FIGS [30]. 


\section{Experience Sampling Method (ESM)}

The Experience Sampling Method is a random time-sampling self-assessment technique; studies have demonstrated the feasibility, validity, and reliability of ESM in general and patient populations [31, 32]. Subjects received a digital wristwatch that emitted a signal ten times a day on six consecutive days, at unpredictable moments between 7:30 a.m. and 10:30 p.m. After each 'beep', subjects completed ESM self-assessment forms concerning current context, thoughts, emotions, and psychotic experiences. Subjects were instructed to complete their reports immediately after the beep, thus minimizing memory distortions. Reports were considered valid when subjects responded within 15 min after the beep, as determined by comparing the actual beep time with the reported time of completion. For inclusion in the analyses, participants had to have provided valid responses to at least one-third of the emitted beeps [33].

\section{Salivary cortisol sampling}

After each ESM beep, subjects collected a saliva sample with a cotton swab (Salivette; Sarstedt, EttenLeur, the Netherlands), replaced the swab in the salivette tube, and recorded the exact collection time. Samples were stored in subjects' home freezers until transport to the lab, where uncentrifuged samples were kept at $-20^{\circ} \mathrm{C}$ until analysis. Saliva samples collected more than 15 minutes after the beep were excluded from the analysis.

\section{ESM measures}

Event stress. Stress was conceptualized in terms of subjective appraisals of events and minor disturbances that continually occur in the natural flow of daily life. After each beep, participants were asked to report the most important event that had happened between the current and the previous report and then to rate this event on a 7-point, bipolar Likert scale $(-3=$ very unpleasant, $0=$ neutral, $3=$ very pleasant). For the current analyses, all positive responses were recoded as 0 , and the negative responses were recoded so that high scores reflect more unpleasant and potentially stressful events $(0=$ neutral, $3=$ very unpleasant) [34].

Psychotic experiences. Psychotic symptomatology was assessed with 8 ESM items rated on 7-point Likert scales ( 1 = not at all to 7 = very): "My thoughts are now paranoid", "My thoughts are difficult to express", "I can't get rid of my thoughts", "My thoughts are influenced by other people", "I feel unreal", "I hear voices", "I see things" and "I am afraid of losing control" [1]. The mean of these 8 ratings formed the variable psychotic experiences. 
Negative affect. Negative affect was assessed as the mean score on 5 ESM items, rated on 7-point Likert scales (1 = not at all to 7 = very): "I feel insecure", "I feel lonely", "I feel anxious", "I feel down" and "I feel guilty".

\section{Salivary cortisol}

Salivary cortisol is a reliable and non-invasive measure of the free, unbound cortisol in blood, which is considered to be the biologically active hormone. Radioimmunoassays were run in duplicate, using a tracer solution of cortisol-3CMO coupled with 2-[125 I] histamine and specific antibodies raised against cortisol-3CMO-BSA [35] (Dr. J. Sulon, Department of Reproductive Physiology, School of Veterinary Medicine, University of Liège). The lower detection limit of the assay was $0.2 \mathrm{nmol} / \mathrm{L}$. The intra- and inter-assay coefficients of variation were $<5 \%$ and $<12 \%$, respectively. All samples from an individual were analyzed in the same assay to reduce sources of variability. Observations with cortisol $>44 \mathrm{nmol} / \mathrm{L}$ $(n=11)$ were considered physiologically abnormal and were excluded from the statistical analysis (van Eck et. al 1996, Peeters et al. 2004, Jacobs et al. 2007).

\section{Questionnaire measures}

Trait psychosis liability. Participants completed the 40-item Community Assessment of Psychic Experiences (CAPE) [36], a self-report instrument that captures variation in the positive and negative dimensions of nonclinical psychotic experiences as well as variation in depression. The total score on the positive dimension (hereafter: CAPE trait score) was used as indicator of psychometric psychosis liability.

Childhood trauma. Participants completed a Dutch version of the Childhood Trauma Questionnaire 25item short form (CTQ) [37]. The CTQ consisted of 25 questions rated on a 5-point Likert scale enquiring about traumatic experiences in childhood. A general measure of childhood trauma was generated by calculating the sum of the answers to all 25 questions.

\section{Statistical analyses}

Multilevel modelling approach. ANOVAs and Chi-square tests were conducted to investigate group differences in demographic characteristics, cross-sectional measures, and mean ratings on ESM variables. ESM and cortisol data were analysed using multilevel regression techniques, which take the hierarchical structure of the data into account. Thirty-five families provided more than one participant, resulting in a further level of clustering. Thus, in the current study, repeated momentary measurements (level 1 ) were clustered within days (level 2) within subjects (level 3), some of whom were members of the same family (level 4). Data were analyzed using the XTMIXED multilevel random regression routine 
in STATA 11.0 [38]. The betas are the fixed regression coefficients of the predictors in the multilevel model. When significant interactions were found, stratified analyses were conducted to clarify group differences, using the STATA LINCOM command to calculate the appropriate linear combinations.

Cortisol values were log transformed to reduce skewness of distribution, generating the variable Incort. The variable time was centered around the grand mean for all samples. To model the cortisol diurnal curve, the variables time and time $e^{2}$ were included as predictors in all analyses with Incort as dependent variable; addition of higher order polynomial terms did not improve model fit. The following confounders were examined in all models: gender, age, recent consumption of food or tobacco use (i.e., in the approximately 90 min interval since the previous ESM beep), use of oral contraceptives, and recent awakening (samples taken within 60 min of awakening).

Cortisol levels and diurnal slope. To test whether mean cortisol level differed between sibling and control groups, a multilevel model was estimated with Incort as the dependent variable and the categorical variable group ( $0=$ controls, $1=$ siblings) as independent variable. To examine whether familial vulnerability for psychosis was reflected in the diurnal cortisol slope, a group by time interaction term was added to the model.

Cortisol reactivity to daily stressors. To investigate whether stress elicited differential cortisol reactions in the two groups, a multilevel analysis was conducted with Incort as the dependent variable and group, event stress and their interaction as the independent variables.

Association between cortisol, psychotic experiences and negative affect. To examine whether increases in psychotic experiences or negative affect were associated with within-person fluctuations in cortisol, we first conducted multilevel analyses with Incort as the dependent variable and group, psychotic experiences (and respectively negative affect) and their interaction as the independent variables. Next, negative affect and psychotic experiences and their interactions with group were included in the same model to determine whether negative affect might mediate the relationship between momentary psychotic experience and cortisol, given that negative emotions have been associated with both cortisol and psychosis [39-41]. 


\section{Results}

\section{Compliance and sample characteristics}

Fourteen participants (11 siblings and 3 control subjects) were unable to comply adequately with the research protocol (13 filled in fewer than 20 valid reports and 1 provided no saliva samples) and were therefore excluded from the analyses. The final sample included 123 participants (60 siblings and 63 controls), who completed a total of 5217 valid ESM observations. Control group participants completed significantly more ESM reports than the siblings (mean (SD) $=45.6$ (8.6) and 41.0 (9.3) reports, respectively; $F=8.31, p=.01$ ). Sample characteristics are summarized in Table 1 . Sibling and control groups were well matched on demographic variables, but controls were on average $4.5 \mathrm{yr}$ older. With the exception of moderate differences in ESM compliance and age, the two groups did not differ significantly from each other on any of the displayed variables.

\section{Cortisol levels and diurnal slope}

Siblings had significantly higher cortisol levels over the ESM sampling moments than controls (Table 2). This pattern was present throughout the day, with no difference between the two groups in the steepness of the diurnal decline in cortisol secretion, as evidenced by the non-significant group by time of day effect shown in Table 2.

\section{Cortisol reactivity to daily stress}

Controls and siblings reported similar frequencies and intensities of daily negative events $($ mean $(S D)=$ 14.5 (12.3) and $13.1(10)$, respectively; $F=0.49, p=0.49$ ). There was no significant interaction between group and event stress in the model of negative affect $\left(\chi^{2}(1)=0.03, p=0.87\right)$, but a main effect of event stress ( $\beta=0.11,95 \% \mathrm{Cl} 0.087,0.122 ; p<0.001)$. Multilevel analysis confirmed the main effect of group on cortisol $(\beta=0.17,95 \% \mathrm{Cl} 0.016,0.316, p=0.03)$ but revealed no main effect of event stress $(\beta=0.01,95 \%$ $\mathrm{Cl}-0.013,0.034 ; \mathrm{p}=0.37)$. However, there was a significant interaction between group and event stress $\left(\chi^{2}(1)=4.11, p=0.04\right)$. Stratified analyses showed an increase in cortisol following unpleasant events in the siblings $(\beta=0.04,95 \% \mathrm{Cl} 0.003,0.075, p=0.03)$, but no such effect in the controls $(\beta=-0.01,95 \% \mathrm{Cl}$ $0.04,0.021 ; p=0.53$ ) (Figure 1). Controlling for negative affect, a putative mediator of the stress-cortisol relationship, had no substantial effect on the results (group by event stress interaction: $\chi^{2}(1)=4.71$, $p=0.03$ ). 


\section{Association between cortisol, psychotic experiences and negative affect}

There was no main effect of psychotic experiences on momentary cortisol. However, group moderated the effect of psychotic experiences on cortisol (Table 3, Model 1). In the sibling group, increased levels of momentary psychotic experiences were associated with increased cortisol levels $(\beta=0.18,95 \% \mathrm{Cl}$ $0.081,0.287 ; p<0.001$ ), whereas in the control group, no association was found between psychotic experiences and cortisol $(\beta=-0.02,95 \% \mathrm{Cl}-0.105,0.075 ; p=0.74)$. For negative affect, there was no main effect in the model predicting cortisol; however an interaction was found with group (Table 3, Model 2). Stratified analyses indicated no significant association between negative affect and cortisol in the control group, whereas in the sibling group cortisol levels increased when participants reported higher negative affect. Finally, in a full model that included group, psychotic experiences, negative affect, and the interactions between group and the two ESM variables, only the interaction between group and psychotic experiences remained significant (Table 3, Model 3).

\section{Post-hoc exploratory analyses}

To better understand the nature of the observed differences in cortisol patterns between sibling and control groups, we conducted a final series of multilevel analyses exploring the possible contribution of depression (presence or absence of lifetime diagnosis), childhood trauma (CTQ total score), overall current stress (mean unpleasantness of daily events during the sampling week) and distress (mean negative affect level) and degree of psychometric psychosis liability (CAPE trait score), by either exploring the interaction with group or adding the appropriate variable as additional predictor in the models of cortisol. Apart from some small effect size alterations, results were similar, with siblings having significantly higher cortisol levels over the ESM sampling moments and a significantly increased cortisol response following unpleasant events compared to controls. Some minor decrease of significance emerged only in the analyses controlling for psychometric psychosis liability (effect of group on cortisol: $\beta=0.15,95 \% \mathrm{Cl}-0.003,0.305 ; p=0.05)$; interaction between group and event stress on cortisol: $\chi^{2}(1)$ $=3.49, \mathrm{p}=0.06)$.

\section{Sensitivity analysis in non-depressed participants}

Additional analyses were carried out, investigating whether diagnoses of depression impacted the results. All analyses were repeated with exclusion of participants who had a current or past diagnosis of depression (controls $n=52$ and relatives $n=48$ remained in the analyses). The results remained the same. 


\section{Discussion}

The current findings indicated that siblings of individuals with psychotic disorder had higher mean cortisol levels throughout the day and greater cortisol reactivity to daily stressors than a comparison group with no family history of psychosis. In the sibling but not the control group, rated intensities of momentary psychotic experiences and negative emotions were associated with transient increases in cortisol secretion. These findings suggest that alterations in HPA axis activity previously reported in patients with established psychotic disorder, unmedicated first-episode patients, and ultra high risk samples may also be present to some degree in healthy first-degree relatives.

The higher diurnal cortisol observed in the sibling group is consistent with reports of elevated HPA activity in patients with schizophrenia [42], first-episode psychosis [9, 17] and individuals at high risk for psychotic disorder [20,43]. However, other studies suggest a blunted cortisol response to stress in chronic and first episode psychosis $[44,45]$. Together, these findings may support the notion that altered activity of the HPA axis may be a marker of underlying vulnerability for psychotic disorder. The finding of greater cortisol reactivity to daily stressors in siblings compared to controls provides new information, in light of the fact that previous studies of neuroendocrine responses to stress in psychotic disorder or at-risk samples were performed in the laboratory. In the control group, we expected to find relatively smaller cortisol stress reactivity to stress compared to the sibling group. In the current study, stressors were daily hassles and minor disturbances in the flow of everyday life. In comparison to experimentally induced stress, these were insufficiently stressful to influence cortisol secretion in our control group, without underlying vulnerability for psychotic disorder. The cortisol non-response in our control group may be a sign of good psychological health, with small daily events not significantly impacting on their stress axis. We have, however, no information about the immediate cortisol response after stressful events and can therefore not eliminate the possibility of an immediate but transient cortisol response with a short recovery time in the control group. Consistent with findings in ESM studies of increased emotional and psychotic symptom reactivity to minor daily stressors in relatives of patients with psychotic disorder $[1,23]$, the current study reveals a similar pattern with respect to cortisol responses in the sibling group. Although siblings did not differ from controls in either the reported frequency or appraised unpleasantness of negative daily events, these minor stressors were more likely to trigger a cortisol increase, supporting the idea that elevated biological stress sensitivity may be a vulnerability marker for psychosis. However, in this sample, siblings and controls did not differ in their emotional reactivity to daily life stress, which contrasts earlier studies in relatives of patients with a psychotic disorder (Myin-Germeys et al. 2001b). Therefore, another possible explanation may be that the current siblings were a relatively healthy subgroup with "normal" levels of emotional stress reactivity. The increased cortisol response to daily hassles may then reflect a protective mechanism.

Another potentially important finding is that momentary increases in psychotic symptomatology were accompanied by increases in cortisol. This association between cortisol and momentary psychotic 
experiences can be interpreted in two causal directions. One plausible interpretation is that the distress associated with psychotic experiences and negative emotions could induce secondary increases in cortisol levels. Several studies have shown that momentary psychotic experiences are related to increases in negative affect $[39,41,46]$; increases in momentary negative affect have, in turn, been linked to heightened cortisol secretion in healthy individuals $[34,47]$. In the current analyses, the estimated group by psychotic experiences interaction effect on cortisol decreased only slightly and remained significant after addition of negative affect and the group by negative affect interaction as predictors. In contrast, there was no significant association between negative affect and cortisol in this complete model. This suggests that sub-clinical psychotic experiences may have made a greater contribution than negative affect to increased cortisol levels in the sibling group. A second interpretation of the observed association between cortisol and momentary psychotic experiences reflects the possibility that increases in cortisol are either directly involved in the pathogenesis of psychotic experiences or reflect secondary, downsteam processes. For instance, cortisol secretion increases dopamine activity in certain brain regions [48]. The dopaminergic system is thought to play an important role in the pathogenesis of positive psychotic symptoms [49]. Thus, heightened cortisol secretion could theoretically, via dopaminergic pathways, increase the likelihood of clinical psychotic experiences. The current finding could be interpreted as evidence for a similar association with subclinical psychotic experiences in unaffected first-degree relatives, but whether this association is causal cannot be established in an ESM study. In general, the observed interrelationships among cortisol, psychotic experiences and negative emotions need to be further explored in longitudinal or experimental designs. Studies that either induce stress and measure subsequent changes in psychotic symptoms and emotions or that induce psychotic experience and subsequent changes in cortisol are needed to answer the question of causality more conclusively.

Dysregulation of the HPA axis is unlikely to be specific for psychosis, as it is found in many disorders, in particular major depression. Moreover, cortisol levels have been found to reflect depression and anxiety, but not psychotic symptoms, in a sample at ultra high risk for psychosis [40]. Post-hoc analyses therefore examined whether a history of depression might explain the results; however, models that controlled for previous depressive episodes yielded similar findings. Alternatively, because siblings share not only genes but also the early rearing environment, childhood adversity - which has been associated with both psychotic disorder [50] and HPA axis abnormalities [51, 52]- could also have influenced the current results. Although the two groups did not differ in self-reported childhood maltreatment, we also conducted post-hoc multilevel analyses to rule out the possibility that group differences in cortisol levels or reactivity were due to differential susceptibility to childhood abuse; these models revealed no association between abuse measures and cortisol in either of the two groups. Another set of analyses examined whether higher cortisol in the siblings might be due to greater overall current stress or distress. This was not the case. Finally, we found that overall psychometric psychosis liability did not significantly influence differences in cortisol patterns between control and sibling groups. Thus, in line with recent findings of cortisol abnormalities in first-episode psychosis that could not be explained by stressful events, perceived stress, or childhood trauma [17], the current results suggest that increased HPA axis activity may indeed reflect the underlying vulnerability to psychosis. The biological processes 
involved, however, remain to be elucidated.

This study has several limitations. First, use of ESM booklets instead of electronic devices means that the exact timing of participants' self- reports and saliva samples cannot be firmly established [53]. However, results of a study comparing self-reported and electronically monitored saliva collection times, with the same intensive, semi-random time-sampling protocol used in the current study, indicated that saliva was generally collected very close to the prescribed time and that self-reported collection times corresponded well with the electronic time-stamps [54]. Another comparative study concluded that paper and electronic diaries yield similar results [55]. Second, no saliva samples were taken at the time of awakening, so that the current dataset does not allow examination of the cortisol awakening response (CAR), a measure of HPA axis activity that appears to be blunted in first-episode psychosis [17]. Third, this study focused solely on the circulating hormone cortisol and can thus provide no insight into underlying mechanisms at higher levels of the HPA axis. Fourth, the interval between cortisol sampling and event occurrence is relevant (van Eck et al. 1996). However, there is no reason to expect systematic differences between groups on the time interval between stressor and time of sampling. Therefore, we are confident that variability in time lag does not question the validity of our analysis on group differences. Moreover, a potentially stressful event could have occurred between 15 minutes and 90 minutes before sampling of cortisol. As the half-life of cortisol is about 1 hour, we still expect to find traces of potential stressors, even when they occurred some time ago. Finally, caution is warranted in generalizing results from the current adult sibling sample to other groups at increased genetic risk for psychosis. Compared to children of parents with a psychotic disorder and others identified as being at ultra high risk for developing a disorder, the siblings included in this study were older, in most cases past the age when onset of psychosis is most likely to occur. The nature and extent of HPA axis abnormalities in healthy siblings may differ from those described in at-risk and ultra high risk adolescent samples. Therefore, it cannot be eliminated that the increased cortisol reactivity in our sibling group reflects a protective rather than a risk factor. On the other hand, it might be that even with a suboptimal biological reactivity, siblings manage to cope better with stress and/or have other resilience factors protecting them from psychotic disorder.

The current study also had some specific strengths. In particular, the repeated sampling of salivary cortisol over 6 days takes into account the well-known but often ignored unreliability of cortisol measures obtained at infrequent intervals [56]. Multiple cortisol measures per person were complemented by a relatively large number of participants. Use of multilevel modeling allowed assessment of within-person associations between cortisol and subjective experience in real time and real-life contexts. Although cortisol measures in both groups were within the normal range, intensive sampling revealed a consistent pattern of heightened HPA activity in the siblings. Taken together, the current findings lend additional credence to the hypothesis that irregularities in HPA axis activity are involved in psychosis vulnerability, broadening the spectrum of vulnerability to include siblings without demonstrable psychometric risk markers. 
Acknowledgments Inez Myin-Germeys was supported by a 2006 NARSAD Young Investigator award and by the Dutch Medical Council (VENI and VIDI grant). This work was also sponsored by the Dutch Organization for Scientific Research NWO (GROUP*). The authors thank research assistants Truda Driesen and Frieda Goethem for their work on this project. 
Table 1. Demographic characteristics and descriptives

\begin{tabular}{|c|c|c|c|c|}
\hline & \multirow{2}{*}{$\begin{array}{l}\text { Controls } \\
(n=63)\end{array}$} & \multirow{2}{*}{$\begin{array}{l}\text { Siblings } \\
(n=60)\end{array}$} & \multicolumn{2}{|c|}{$\begin{array}{c}\text { Group } \\
\text { Comparisons }\end{array}$} \\
\hline & & & $\begin{array}{l}\text { Test } \\
\text { statistic }\end{array}$ & $\mathrm{p}$-value \\
\hline Age, years: mean (SD) & $33.27(10.3)$ & $28.82(10.0)$ & $F=5.93$ & 0.02 \\
\hline Gender (male:female) & $18: 45$ & $22: 38$ & $\chi^{2}(1)=0.98$ & 0.34 \\
\hline Education, $\mathrm{n}(\%)^{*}$ & & & $\chi^{2}(1)=2.53$ & 0.11 \\
\hline Secondary school or less & $20(31.8 \%)$ & $27(45.8 \%)$ & & \\
\hline Higher education & $43(68.3 \%)$ & $32(54.2 \%)$ & & \\
\hline Marital status, $\mathrm{n}(\%)^{*}$ & & & $\chi^{2}(1)=1.31$ & 0.25 \\
\hline Married or living together & 39 (61.9\%) & $31(51.7 \%)$ & & \\
\hline Never married/single/divorced & $24(38.2 \%)$ & 29 (48.4\%) & & \\
\hline Work situation, $\mathrm{n}(\%)^{*}$ & & & $\chi^{2}(1)=1.34$ & 0.51 \\
\hline $\begin{array}{l}\text { Working/significant housework/ } \\
\text { studying }\end{array}$ & $61(98.8 \%)$ & $58(96.7 \%)$ & & \\
\hline Disabled or unemployed & $2(3.2 \%)$ & $2(3.3 \%)$ & & \\
\hline Living situation, $\mathrm{n}(\%)^{*}$ & & & $\chi^{2}(4)=7.85$ & 0.10 \\
\hline Alone & 7 (11.1\%) & $3(5 \%)$ & & \\
\hline With partner/family/children & $43(68.3 \%)$ & $33(55 \%)$ & & \\
\hline With parents/relatives & $10(15.9 \%)$ & $20(33.3 \%)$ & & \\
\hline Other & $3(4.8 \%)$ & $4(6.7 \%)$ & & \\
\hline $\mathrm{CASH}^{1} \mathrm{DSM}$-IV Axis I diagnosis lifetime, $\mathrm{n}(\%)^{*}$ & & & $\chi^{2}(2)=1.56$ & 0.46 \\
\hline Depressive disorder, in partial remission & $3(4.8 \%)$ & $1(1.7 \%)$ & & \\
\hline Depressive disorder, in full remission & $8(12.7 \%)$ & $11(18.3 \%)$ & & \\
\hline No diagnosis & $52(82.5 \%)$ & $48(80 \%)$ & & \\
\hline CAPE trait score, mean (SD) & $1.0(0.14)$ & $1.1(0.21)$ & $F=2.04$ & 0.16 \\
\hline CTQ abuse, total score (SD) & $33.13(7.71)$ & $33.57(8.43)$ & $F=0.09$ & 0.76 \\
\hline Current smoker (n:y) & 49:14 & $43: 17$ & $\chi^{2}(1)=0.61$ & 0.44 \\
\hline Current cannabis user ( $\mathrm{n}: \mathrm{y})$ & $61: 2$ & $57: 3$ & $\chi^{2}(1)=0.26$ & 0.61 \\
\hline \multicolumn{5}{|l|}{ ESM variables } \\
\hline Event stress, mean ${ }^{2}(\mathrm{SD})$ & 0.21 & 0.17 & $F=1.38$ & 0.24 \\
\hline Psychotic experiences, mean $^{2}$ (SD) & $1.1(0.16)$ & $1.1(0.17)$ & $F=0.09$ & 0.78 \\
\hline Negative affect, mean $^{2}$ (SD) & $1.23(0.28)$ & $1.21(0.34)$ & $F=0.09$ & 0.76 \\
\hline Momentary cortisol, mean ${ }^{2} \mathrm{nmol} / \mathrm{L}$ (SD) & $2.91(0.15)$ & $3.56(0.22)$ & $F=5.98$ & 0.02 \\
\hline
\end{tabular}

*Due to rounding, percentages may not add exactly to $100 \%$

${ }^{1}$ Comprehensive Assessment of Symptoms and History (CASH) [28]

${ }^{2}$ For the Experience Sampling variables, an individual mean was first calculated over all reports; these values were then aggregated to obtain the group mean and SD. 
Table 2. Multilevel regression estimates for effects of group on cortisol level and diurnal slope

\begin{tabular}{llllll}
\hline & $\beta$ & $95 \% \mathrm{Cl}$ & $\mathrm{SE}$ & $\mathrm{Z}$ & $p$ \\
\hline Intercept & 1.137 & $0.780,1.494$ & 0.182 & 6.25 & $<0.001$ \\
Group & 0.169 & $0.019,0.319$ & 0.076 & 2.21 & 0.027 \\
$\begin{array}{l}\text { Time of day } \\
\text { (diurnal slope) }\end{array}$ & -0.130 & $-0.134,-0.125$ & 0.002 & -54.43 & $<0.001$ \\
Group x Time of day & -0.003 & $-0.009,0.004$ & 0.003 & -0.85 & 0.393 \\
Age & -0.009 & $-0.017,-0.002$ & 0.004 & -2.55 & 0.011 \\
Gender & -0.181 & $-0.338,-0.024$ & 0.079 & -2.27 & 0.023 \\
Oral contraceptives & 0.155 & $-0.015,0.325$ & 0.087 & 1.79 & 0.074 \\
Recent awakening & 0.330 & $0.269,0.391$ & 0.031 & 10.63 & $<0.001$ \\
Recent food intake & 0.161 & $0.128,0.193$ & 0.016 & 9.77 & $<0.001$ \\
Recent smoking & 0.124 & $0.045,0.203$ & 0.040 & 3.08 & 0.002 \\
\hline
\end{tabular}

Note. The dependent variable is log-transformed cortisol (Incort). Regression coefficients are unstandardized. Group is coded 1 for siblings and 0 for controls. The variable Time of day is centered around the grand mean. Time $^{2}$ was also a significant predictor of Incort $(\mathrm{p}<0.001)$ and is controlled for in all analyses. Recent awakening refers to samples taken within $60 \mathrm{~min}$ after awakening; recent food intake and smoking refer to reported occurrence of these activities in the interval between two ESM reports (roughly $90 \mathrm{~min}$ ).

Table 3. Multilevel estimates of the effects of momentary psychotic experiences and negative affect on cortisol, as moderated by group (siblings versus controls)

\begin{tabular}{llll}
\hline & \multicolumn{2}{c}{$\beta(\mathrm{SE})$} \\
\cline { 2 - 4 } & Model 1 & Model 2 & Model 3 \\
\hline Intercept & $1.12(0.19)^{* * *}$ & $1.09(0.18)^{* * *}$ & $1.10(0.19)^{* * *}$ \\
Group & $-0.05(0.11)$ & $0.05(0.09)$ & $-0.08(0.11)$ \\
Negative affect & & $0.02(0.03)$ & $0.02(0.03)$ \\
Group x Negative affect & & $0.10(0.04)^{* *}$ & $0.07(0.04)$ \\
Psychotic experience & $-0.02(0.05)$ & & $-0.03(0.5)$ \\
Group x Psychotic experience & $0.20(0.07)^{* *}$ & & $0.15(0.08)^{*}$
\end{tabular}

Note. The dependent variable is Incort. The models control for time, time ${ }^{2}$, age, gender, oral contraceptive use, recent awakening, recent food intake and recent smoking. $\beta=$ unstandardized regression coefficient; SE = Standard error; $* * * p<.001, * * p<.01, * p<.05$ 
Figure 1: Cortisol reactivity to stressful events

Modelled change (based on regression coefficient) in untransformed cortisol values ( $\mathrm{nmol} / \mathrm{L})$

following daily events, according to their appraised unpleasantness ( $0=$ neutral, $3=$ very unpleasant), in sibling and control groups.

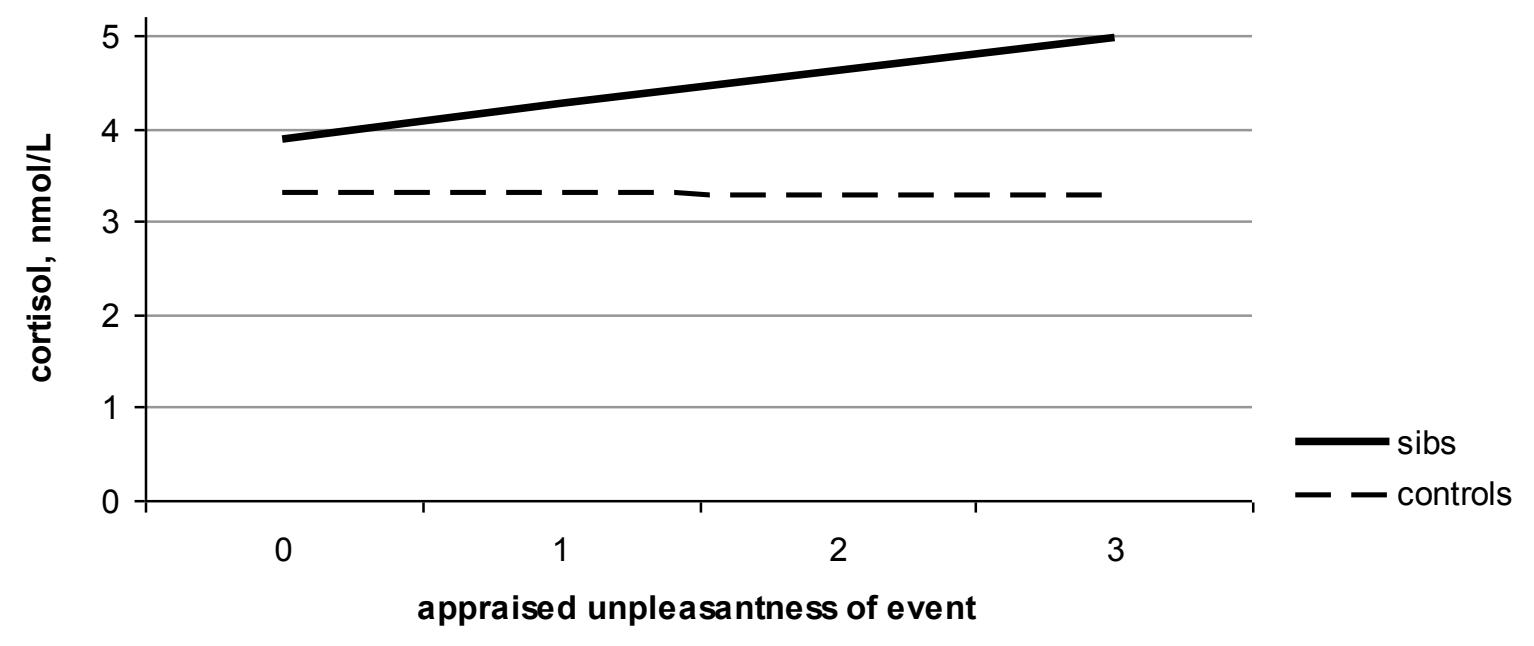




\section{References}

1. Myin-Germeys I, Delespaul P, and van Os J (2005): Behavioural sensitization to daily life stress in psychosis. Psychological Medicine 35:733-41.

2. Myin-Germeys I, Marcelis M, Krabbendam L, Delespaul P, and van Os J (2005): Subtle fluctuations in psychotic phenomena as functional states of abnormal dopamine reactivity in individuals at risk. Biological Psychiatry 58:105-10.

3. Soliman A, O'Driscoll GA, Pruessner J, Holahan AL, Boileau I, Gagnon D, et al. (2008): Stress-induced dopamine release in humans at risk of psychosis: a [11C]raclopride PET study. Neuropsychopharmacology 33:2033-41.

4. van Winkel R, Stefanis NC, and Myin-Germeys I (2008): Psychosocial stress and psychosis. A review of the neurobiological mechanisms and the evidence for gene-stress interaction. Schizophrenia Bulletin 34:1095-105.

5. Walker E, Mittal V, and Tessner K (2008): Stress and the hypothalamic pituitary adrenal axis in the developmental course of schizophrenia. Annual Review of Clinical Psychology 4:189-216.

6. Koolschijn PC, van Haren NE, Hulshoff Pol HE, and Kahn RS (2008): Hypothalamus volume in twin pairs discordant for schizophrenia. European Neuropsychopharmacology 18:312-5.

7. Pariante CM (2008): Pituitary volume in psychosis: the first review of the evidence. Journal of Psychopharmacology 22:76-81.

8. Wright IC, Rabe-Hesketh S, Woodruff PW, David AS, Murray RM, and Bullmore ET (2000): Meta-analysis of regional brain volumes in schizophrenia. American Journal of Psychiatry 157:16-25.

9. Ryan MC, Sharifi N, Condren R, and Thakore JH (2004): Evidence of basal pituitary-adrenal overactivity in first episode, drug naive patients with schizophrenia. Psychoneuroendocrinology 29:1065-70.

10. Jansen LM, Gispen-de Wied CC, Gademan PJ, De Jonge RC, van der Linden JA, and Kahn RS (1998): Blunted cortisol response to a psychosocial stressor in schizophrenia. Schizophrenia Research 33:87-94.

11. Jansen LM, Gispen-de Wied CC, and Kahn RS (2000): Selective impairments in the stress response in schizophrenic patients. Psychopharmacology (Berlin) 149:319-25.

12. Marcelis M, Cavalier E, Gielen J, Delespaul P, and Van Os J (2004): Abnormal response to metabolic stress in schizophrenia: marker of vulnerability or acquired sensitization? Psychological Medicine 34:1103-11.

13. Breier A, Wolkowitz OM, Doran AR, Bellar S, and Pickar D (1988): Neurobiological effects of lumbar puncture stress in psychiatric patients and healthy volunteers. Psychiatry Research 25:187-94.

14. Albus M, Ackenheil M, Engel RR, and Muller F (1982): Situational reactivity of autonomic functions in schizophrenic patients. Psychiatry Research 6:361-70.

15. Brenner K, Liu A, Laplante DP, Lupien S, Pruessner JC, Ciampi A, et al. (2009): Cortisol response to a psychosocial stressor in schizophrenia: blunted, delayed, or normal? Psychoneuroendocrinology 34:859-68.

16. Walsh P, Spelman L, Sharifi N, and Thakore JH (2005): Male patients with paranoid schizophrenia have greater ACTH and cortisol secretion in response to metoclopramide-induced AVP release. Psychoneuroendocrinology 30:431-7.

17. Mondelli V, Dazzan P, Hepgul N, Di Forti M, Aas M, D’Albenzio A, et al. (2010): Abnormal cortisol levels during the day and cortisol awakening response in first-episode psychosis: the role of stress and of antipsychotic treatment. Schizophrenia Research 116:234-42.

18. Wik G (1995): Effects of neuroleptic treatment on cortisol and 3-methoxy-4-hydroxyphenylethyl glycol levels in blood. Journal of Endocrinology 144:425-9.

19. Meltzer HY, Koenig JI, Nash JF, and Gudelsky GA (1989): Melperone and clozapine: neuroendocrine effects of atypical neuroleptic drugs. Acta Psychiatrica Scandinavica Suppl 352:24-9. 
20. Mittal VA, Dhruv S, Tessner KD, Walder DJ, and Walker EF (2007): The relations among putative biorisk markers in schizotypal adolescents: minor physical anomalies, movement abnormalities, and salivary cortisol. Biological Psychiatry 61:1179-86.

21. Walker EF, Walder DJ, and Reynolds F (2001): Developmental changes in cortisol secretion in normal and at-risk youth. Development and Psychopathology 13:721-32.

22. Garner B, Pariante CM, Wood SJ, Velakoulis D, Phillips L, Soulsby B, et al. (2005): Pituitary volume predicts future transition to psychosis in individuals at ultra-high risk of developing psychosis. Biological Psychiatry 58:417-23.

23. Myin-Germeys I, van Os J, Schwartz JE, Stone AA, and Delespaul PA (2001): Emotional reactivity to daily life stress in psychosis. Archives of General Psychiatry 58:1137-44.

24. Mondelli V, Dazzan P, Gabilondo A, Tournikioti K, Walshe M, Marshall N, et al. (2008): Pituitary volume in unaffected relatives of patients with schizophrenia and bipolar disorder. Psychoneuroendocrinology 33:1004-12.

25. Goldstein JM, Seidman LJ, Makris N, Ahern T, O’Brien LM, Caviness VS, Jr., et al. (2007): Hypothalamic abnormalities in schizophrenia: sex effects and genetic vulnerability. Biological Psychiatry 61:935-45.

26. Nicolson N (2007): Measurement of cortisol, in Handbook of Physiological Research Methods in Health Psychology, LJ LL, Gallo, Editor. 2007, Sage Publications: Thousand Oaks, CA. p. 37-74.

27. G.R.O.U.P. (2010): Evidence that Familial Liability for Psychosis is Expressed as Differential Sensitivity to Cannabis: an Analysis of Patient-Sibling and Sibling-Control Pairs. Archives of General Psychiatry in press.

28. Andreasen NC, Flaum M, and Arndt S (1992): The Comprehensive Assessment of Symptoms and History (CASH). An instrument for assessing diagnosis and psychopathology. Archives of General Psychiatry 49:615-23.

29. APA (1994): Diagnostic and Statistical Manual of Mental Disorders, Fourth Edition. 1994, Washington, DC: American Psychiatric Association.

30. NIMH.Genetics.Initiative. (1992): Family Interview for Genetic Studies (FIGS). 1992, Rockville, Md: National Institute of Mental Health.

31. Csikszentmihalyi M and Larson R (1987): Validity and reliability of the Experience-Sampling Method. Journal of Nervous and Mental Disease 175:526-36.

32. Myin-Germeys I, Oorschot M, Collip D, Lataster J, Delespaul P, and van Os J (2009): Experience sampling research in psychopathology: opening the black box of daily life. Psychological Medicine 39:1533-1547.

33. Delespaul P, deVries M, and van Os J (2002): Determinants of occurrence and recovery from hallucinations in daily life. Social Psychiatry and Psychiatric Epidemiology 37:97-104.

34. Jacobs N, Myin-Germeys I, Derom C, Delespaul P, van Os J, and Nicolson NA (2007): A momentary assessment study of the relationship between affective and adrenocortical stress responses in daily life. Biological Psychology 74:60-6.

35. Sulon J, Demey-Ponsart L, Beauduin P, and Sodoyez JC (1978): Radioimmunoassay of corticosterone, cortisol and cortisone: their application to human cord and maternal plasma. Journal of Steroid Biochemistry and Molecular Biology 9:671-6.

36. Hanssen M, Bak M, Bijl R, Vollebergh W, and van Os J (2005): The incidence and outcome of subclinical psychotic experiences in the general population. British Journal of Clinical Psychology 44:181-91.

37. Bernstein DP, Stein JA, Newcomb MD, Walker E, Pogge D, Ahluvalia T, et al. (2003): Development and validation of a brief screening version of the Childhood Trauma Questionnaire. Child Abuse \& Neglect 27:169-90.

38. StataCorp (2009): STATA/SE statistical software, release 11. 2009, Texas: College Station.

39. Freeman D and Garety PA (2003): Connecting neurosis and psychosis: the direct influence of emotion on delusions and hallucinations. Behavioral Research and Therapy 41:923-47.

40. Thompson KN, Phillips LJ, Komesaroff P, Yuen HP, Wood SJ, Pantelis C, et al. (2007): Stress and HPA-axis functioning in young people at ultra high risk for psychosis. Journal of Psychiatric Research 41:561-9. 
41. Myin-Germeys I, Nicolson NA, and Delespaul PA (2001): The context of delusional experiences in the daily life of patients with schizophrenia. Psychological Medicine 31:489-98.

42. Muck-Seler D, Pivac N, Mustapic M, Crncevic Z, Jakovljevic M, and Sagud M (2004): Platelet serotonin and plasma prolactin and cortisol in healthy, depressed and schizophrenic women. Psychiatry Research 127:217-26.

43. Walker EF, Brennan PA, Esterberg M, Brasfield J, Pearce B, and Compton MT (2010): Longitudinal changes in cortisol secretion and conversion to psychosis in at-risk youth. Journal of Abnormal Psychology 119:401-8.

44. Jansen LM, Gispen-de Wied CC, and Kahn RS (2000): Selective impairments in the stress response in schizophrenic patients. Psychopharmacology (Berl) 149:319-25.

45. van Venrooij JA, Fluitman SB, Lijmer JG, Kavelaars A, Heijnen CJ, Westenberg HG, et al. (2010): Impaired Neuroendocrine and Immune Response to Acute Stress in Medication-Naive Patients With a First Episode of Psychosis. Schizophr Bull.

46. Thewissen V, Bentall RP, Oorschot M, Campo JA, van Lierop T, van Os J, et al. (2010): Emotions, self-esteem, and paranoid episodes: An experience sampling study. The British Journal of Clinical Psychology DOI: 10.1348/014466 $510 \times 508677$.

47. van Eck M, Berkhof H, Nicolson N, and Sulon J (1996): The effects of perceived stress, traits, mood states, and stressful daily events on salivary cortisol. Psychosomatic Medicine 58:447-58.

48. Dallman MF, Akana SF, Strack AM, Scribner KS, Pecoraro N, La Fleur SE, et al. (2004): Chronic stress-induced effects of corticosterone on brain: direct and indirect. Annals of the New York Academy of Sciences 1018:141-50.

49. Laruelle M and Abi-Dargham A (1999): Dopamine as the wind of the psychotic fire: new evidence from brain imaging studies. Journal of Psychopharmacology 13:358-71.

50. Read J, van Os J, Morrison AP, and Ross CA (2005): Childhood trauma, psychosis and schizophrenia: a literature review with theoretical and clinical implications. Acta Psychiatrica Scandinavica 112:330-50.

51. De Bellis MD, Chrousos GP, Dorn LD, Burke L, Helmers K, Kling MA, et al. (1994): Hypothalamic-pituitary-adrenal axis dysregulation in sexually abused girls. Journal of Clinical Endocrinology \& Metabolism 78:249-55.

52. Tarullo AR and Gunnar MR (2006): Child maltreatment and the developing HPA axis. Hormones and Behavior 50:632-9.

53. Stone AA, Shiffman S, Schwartz JE, Broderick JE, and Hufford MR (2002): Patient non-compliance with paper diaries. British Medical Journal 324:1193-4.

54. Jacobs N, Nicolson NA, Derom C, Delespaul P, van Os J, and Myin-Germeys I (2005): Electronic monitoring of salivary cortisol sampling compliance in daily life. Life Sciences 76:2431-43.

55. Green AS, Rafaeli E, Bolger N, Shrout PE, and Reis HT (2006): Paper or plastic? Data equivalence in paper and electronic diaries. Psychological Methods 11:87-105.

56. Hruschka DJ, Kohrt BA, and Worthman CM (2005): Estimating between- and within-individual variation in cortisol levels using multilevel models. Psychoneuroendocrinology 30:698-714. 


\footnotetext{
${ }^{1}$ Dept. of Psychiatry and Psychology, School for Mental Health and Neuroscience, EURON, Maastricht University Medical Centre, PO Box 616 (Vijv1), 6200 MD Maastricht, The Netherlands

${ }^{2}$ King's College London, King's Health Partners, Department of Psychosis Studies Institute of Psychiatry, London, UK

* contributed equally
} 


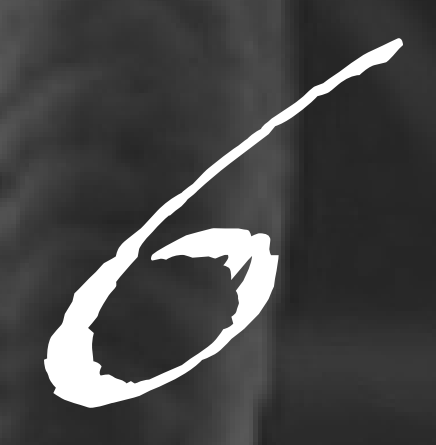

\section{Hippocampal Volume as Marker of Daily Life Emotional and Cortisol Stress Sensitivity in Psychosis}

Dina Collip ${ }^{1 *}$, Petra Habets ${ }^{1 *}$, Machteld Marcelis ${ }^{1}$, Ed Gronenschild ${ }^{1}$, Tineke Lataster ${ }^{1}$, Marielle Lardinois $^{1}$, Nancy A. Nicolson ${ }^{1}$, Jim van Os ${ }^{1-2}$, and Inez Myin-Germeys ${ }^{1}$ for G.R.O.U.P. 


\section{Abstract}

\section{Background}

Reduced hippocampal size and increased stress sensitivity are associated with genetic risk for psychotic disorder. However, to what degree the hippocampus is implicated in daily life stress reactivity in psychotic disorder has never been examined. The current study investigated (i) whether hippocampal volume (HV) was associated with emotional and cortisol daily stress reactivity, and (ii) whether genetic risk (the contrast between controls, patients and siblings of patients) moderated this relationship.

\section{Methods}

T1-weighted MRI scans were acquired from 20 patients with schizophrenia, 38 healthy siblings at higher than average genetic risk for schizophrenia and 33 controls. Freesurfer 5.0.0 was used to measure HV. The Experience Sampling Method (ESM; a structured momentary assessment diary technique) was used to assess emotional stress reactivity (the effect of momentary stress on momentary negative affect (NA)). Cortisol stress reactivity was assessed using momentary cortisol levels extracted from saliva in the control and sibling groups.

\section{Results}

Multilevel linear regression analyses revealed a significant three-way interaction between group, HV, and momentary stress in both the model of NA and the model of cortisol. Increased emotional stress reactivity was associated with smaller left HV in patients and larger total HV in controls. In line with the results in patients, siblings with small HV demonstrated increased emotional and cortisol stress reactivity compared to those with large HV.

\section{Conclusions}

The findings suggest that HV may index risk and possibly disease-related mechanisms underlying daily life emotional and cortisol stress reactivity in psychotic disorder. 


\section{Introduction}

Patients with psychotic disorder generally display reduced hippocampal size [1-4]. Recent studies have shown that similar alterations in hippocampal volume (HV) may be present in first-degree relatives of patients with schizophrenia [5, 6], suggesting that HV alterations constitute part of the liability to psychosis. The hippocampus plays a pivotal role in regulating emotional responses to stressful stimuli and in the negative feedback mechanism controlling hypothalamic-pituitary-adrenal (HPA) axis activity [7-11]. Because the hippocampus has an inhibitory influence, a smaller HV might be associated with increased HPA axis reactivity to stress. Indeed, in clinical samples, studies have reported an inverse association between HV and cortisol levels. In two studies, hippocampal atrophy was accompanied by hypercortisolemia $[12,13]$. Similarly, in patients with a psychotic disorder, smaller left HV was associated with higher salivary cortisol levels [14].

In contrast, findings in healthy volunteers show mixed results. One study, examining the association between HV and cortisol response to experimental psychological stress as well as awakening [15], found a positive association between HV and cortisol reactivity in young healthy volunteers, whereas a negative association between $\mathrm{HV}$ and the cortisol response to a physiological challenge was found in another sample [16]. These findings indicate that the association between HV and stress reactivity may be different in healthy and clinical populations.

Familial vulnerability for psychosis has been associated with amplified emotional and cortisol responses to stress in daily life. Patients with a psychotic disorder and their unaffected first-degree relatives have been found to display increased negative emotions and an increase in psychotic experiences $[17,18]$. Similarly, a recent study showed that siblings of patients compared to controls respond with increased cortisol secretion to minor everyday stressors (Collip et al., in press). The neural mechanisms underlying this augmented stress sensitivity, however, have not yet been identified. The current study investigates whether HV is associated with emotional and cortisol reactivity to daily life stress, as indexed by momentary variation in negative affect and momentary cortisol levels. Given the reported discrepancy between clinical and non-clinical samples in the association between HV and cortisol [19], we examined whether this association was moderated by genetic risk for psychosis. Because some earlier studies suggest that cortisol levels are primarily associated with left HV [14], analyses also investigated hemispheric differentiation: differences in the HV stress reactivity. Analyses thus examined to what degree (i) HV was associated with emotional and cortisol reactivity to daily stress, and (ii) group (control, sibling, patient) moderated the association between (emotional and cortisol) reactivity to stress and HV. 


\section{Hippocampal volume as marker of daily life stress sensitivity in psychosis}

\section{Methods and Materials}

\section{Subjects}

The sample included patients with a diagnosis of non-affective psychotic disorder, their siblings, and controls from the general population, in the context of the Dutch national GROUP project [20]. In selected representative geographical areas in the Netherlands and Belgium, patients were identified through clinicians whose caseload was screened for inclusion criteria. Subsequently, a group of patients presenting consecutively at these services either as outpatients or inpatients, were recruited for the study. First-degree relatives were recruited through participating patients. Control subjects were recruited from the same population as the patients, through random mailings in the geographic region and advertisements in newspapers. All interviews were conducted by trained psychology graduates. From the wider sampling frame, the following subgroups were selected: 20 patients with a diagnosis of non-affective psychotic disorder, 37 siblings of patients with a diagnosis of schizophrenia and 32 controls. Inclusion criteria were: (i) age 16 to 50 years, (ii) diagnosis of non-affective psychotic disorder and (iii) good command of the Dutch language. Siblings had to be free of any lifetime non-affective psychotic disorder. For the control subjects, the occurrence of any psychotic disorder in either the subject or any first-degree family member, assessed using the Family Interview for Genetic Studies (FIGS) [21], constituted an exclusion criterion.

Diagnosis was based on the Diagnostic and Statistical Manual of Mental Disorder-IV (DSM-IV) criteria [22], assessed with the Comprehensive Assessment of Symptoms and History (CASH) interview [23]. Patients were diagnosed with: schizophrenia $(n=11)$, schizoaffective disorder $(n=2)$, schizophreniform disorder $(n=1)$, brief psychotic disorder $(n=2)$, and psychotic disorder not otherwise specified $(n=4)$. The CASH was also used to confirm the absence of a diagnosis of non-affective psychosis in the siblings, and absence of a lifetime diagnosis of any psychotic disorder or any current affective disorder in the healthy controls. Six siblings and seven controls had a history of major depressive disorder, but none presented in a current depressive episode.

Prior to MRI acquisition, participants were screened for the following exclusion criteria: (i) brain injury with unconsciousness for over 5 minutes, (ii) meningitis or other neurological diseases that might have affected brain structure/function, (iii) respiratory or cardiac disease and (iv) severe claustrophobia. In addition, subjects with metal corpora aliena were excluded from the study, as well as women with an intrauterine device or (suspected) pregnancy. The study was approved by the standing ethics committee, and all the subjects gave written informed consent in accordance with the committee's guidelines.

\section{Measures}

The Positive and Negative Syndrome Scale (PANSS) [24] was used to measure psychotic symptoms over the last two weeks. 
Antipsychotic medication was determined from reports of the participant's psychiatrist. Best estimate lifetime (cumulative) antipsychotic medication (AP) use was determined by multiplying the number of days of AP use with the corresponding haloperidol equivalents and summing up these scores for all periods of AP use [25].

Estrogen exposure was estimated by multiplying the number of months of oral contraceptive use with micrograms estrogen (of the corresponding oral contraceptive) per month.

Substance use was assessed using the Composite International Diagnostic Interview (CIDI) sections B-J-L [26]. Cannabis use and other drug use (stimulants, sedatives, opiates, cocaine, PCP, psychedelics, inhalants, or other (e.g., XTC, poppers)) was assessed as reported frequency of use (i) during the last 12 months, and (ii) lifetime. Alcohol and tobacco use was defined as the reported number of weekly consumptions during the last 12 months.

\section{Experience Sampling Method (ESM)}

The Experience Sampling Method is a random time-sampling self-assessment technique; studies have demonstrated the feasibility, validity, and reliability of ESM in general and patient populations [27, 28]. Subjects received a digital wristwatch that emitted a signal ten times a day on six consecutive days, at unpredictable moments between 7:30 a.m. and 10:30 p.m. After each 'beep', subjects completed ESM self-assessment forms concerning current context, thoughts, emotions, and psychotic experiences. Subjects were instructed to complete their reports immediately after the beep, thus minimizing memory distortions. Reports were considered valid when subjects responded within 15 min after the beep, as determined by comparing the actual beep time with the reported time of completion. For inclusion in the analyses, participants had to have provided valid responses to at least one-third of the emitted beeps [29].

Stress reactivity, as described in previous work, was conceptualized as emotional [18] and cortisol reactivity (Collip et al., in press)[30] to minor disturbances in daily life. Momentary measures of stress, negative affect (NA) and cortisol were derived from the experience sampling data as described below.

\section{ESM measures}

Event stress. In accordance with previous work, stress was conceptualized as the subjectively appraised unpleasantness of distinctive events [18]. After each beep, participants were asked to report the most important event that had happened between the current and the previous report and then to rate this event on a 7-point scale (-3 = very unpleasant, $0=$ neutral, $3=$ very pleasant). For the current analyses, all positive responses were recoded as 0 , and the negative responses were recoded so that high scores reflect more unpleasant and potentially stressful events ( 0 = neutral, $3=$ very unpleasant $)$ [31]. 
Negative affect. In line with previous reports [18] ESM NA was assessed as the mean score on 6 ESM items, rated on 7-point Likert scales (1 = not at all to 7 = very): "I feel insecure", "I feel lonely", "I feel anxious", "I feel down", "I feel guilty" and "I feel angry/ irritated" (Cronbach's $\alpha=.84)$.

\section{Salivary cortisol sampling}

After each ESM beep, participants (siblings, controls) collected a saliva sample with a cotton swab (Salivette; Sarstedt, Etten-Leur, the Netherlands), replaced the swab in the salivette tube, and recorded the exact collection time. Samples were stored in subjects' home freezers until transport to the lab, where uncentrifuged samples were kept at $-20^{\circ} \mathrm{C}$ until analysis. Saliva samples collected more than 15 minutes after the beep were excluded from the analysis.

\section{Salivary cortisol}

Salivary cortisol is a reliable and non-invasive measure of the free, unbound cortisol in plasma, the biologically active hormone. Radioimmunoassays were run in duplicate, using a tracer solution of cortisol-3CMO coupled with 2-[125I] histamine and specific antibodies raised against cortisol-3CMO-BSA [31] (Dr. J. Sulon, Department of Reproductive Physiology, School of Veterinary Medicine, University of Liège). The lower detection limit of the assay was $0.2 \mathrm{nmol} / \mathrm{L}$. The intra- and inter-assay coefficients of variation were $<5 \%$ and $<12 \%$, respectively. All samples from an individual were analyzed in the same assay to reduce sources of variability. All samples were within the normal physiological range $(<$ $44 \mathrm{nmol} / \mathrm{L}$ ). Cortisol was not measured in the patients with a psychotic disorder, as they usually receive antipsychotic medication, which can affect cortisol levels.

\section{MRI acquisition and processing}

MRI scans were acquired using a 3T Siemens scanner and the following acquisition parameters: Modified Driven Equilibrium Fourier Transform (MDEFT) sequence: 176 slices, $1 \mathrm{~mm}$ isotropic voxel size, TE 2.4 ms, TR $7.92 \mathrm{~ms}$, flip angle $15^{\circ}$, total acquisition time: $12 \mathrm{~min}$ 51s, and for the Magnetization Prepared Rapid Acquisition Gradient Echo (MP-RAGE): Alzheimer's Disease Neuroimaging Initiation (ADNI) sequence: 192 slices, $1 \mathrm{~mm}$ isotropic voxel size, TE $2.6 \mathrm{~ms}$, TR $2250 \mathrm{~ms}$, flip angle $9^{\circ}$, total acquisition time: $7 \mathrm{~min} 23 \mathrm{~s}$. For both sequences the matrix size and field of view were 256x256. Two sequences were used because of a scanner update during data collection. The MP-RAGE and MDEFT are very similar, but to prevent any systematic bias, the total proportion of MP-RAGE scans (around 1/3) was balanced between the groups.

\section{MRI preprocessing}

Scans were processed and analyzed using Freesurfer stable release v5.0.0. Technical details of these procedures are described in prior publications [32-38]. Data were automatically transformed into Talairach standard space. 


\section{Volume measures}

The automated procedures for volumetric measures of the different brain structures are described by Fischl et al. This procedure automatically assigns a neuroanatomical label to each voxel in an MRI volume based on probabilistic information automatically estimated from a manually labelled training set [see 34 for detailed description]. The training set included both healthy persons in the age range 1887 years and a group of Alzheimer's disease patients in the age range 60-87 years, and the classification technique employs a registration procedure that is robust to anatomical variability, including the ventricular enlargement typically associated with neurological diseases and aging. The technique has previously been shown to be comparable in accuracy to manual labelling. The segmentations were visually inspected for accuracy.

\section{Statistical analyses}

ESM and cortisol data were analysed using multilevel regression techniques, which take the hierarchical structure of the data into account. In the current study, repeated momentary measurements (level 1) were nested in subjects (level 2) who were part of the same family (level 3). Data were analyzed using the XTMIXED multilevel random regression routine in STATA 11.0 [39]. Effect sizes from predictors in the multilevel model were expressed as $B$, representing the unstandardized fixed regression coefficient. Interactions were assessed by Wald test. The size of the moderator effects was calculated by applying and testing the appropriate linear combinations using the STATA MARGIN command. Raw cortisol values were log transformed to reduce skewness of distribution, generating the variable Incort. The variable time was centered around the grand mean for all samples. To model the cortisol diurnal curve, the variable time was included as a predictor in all analyses with Incort as dependent variable; addition of higher order polynomial terms did not improve model fit. Analyses were adjusted for the $a$ priori selected confounders age, sex, intracranial volume, and scan type. In the patient group AP medication use on HV size was examined. Analyses were re-run including AP [14, 40], antidepressant medication and history of depression.

\section{Group differences in hippocampal volume and cortisol}

To test whether HV or mean cortisol level differed between groups, a regression was estimated with HV and Incort respectively as the dependent variable and the categorical variable group $(0=$ controls, $1=$ siblings, 2=patients; for cortisol, controls and siblings only) as independent variable.

\section{Interaction between group, stress reactivity and volume}

In order to test whether HV was associated with emotional stress reactivity, and whether this was 
moderated by group, regression analyses were conducted with event stress, HV and group (entered as linear three-level variable of patients, siblings and controls = reference) as well as their interaction terms as independent variables and NA as the dependent variable

$$
\begin{aligned}
& \left.\left.N A=B_{0}+B_{1} \text { (group) }+B_{2} \text { (stress }\right)+B_{3}(\text { volume })+B_{4}(\text { group } \times \text { stress })+B_{5} \text { (group } \times \text { volume }\right) \\
& \left.+B_{6}(\text { stress } \times \text { volume })+B_{7} \text { (group } \times \text { stress } \times \text { volume }\right) .
\end{aligned}
$$

For visualization purposes, HV was entered as dummy variable representing the distribution of volume calculated at the $50^{\text {th }}$ percentiles of $\mathrm{HV}$ of the controls $\left(1=<50^{\text {th }}\right.$ percentile: small $\mathrm{HV}, 2=>50^{\text {th }}$ percentile: large HV). To ensure that definitions for small and large HV were the same for all groups, patients and siblings were allocated to hippocampal group on the basis of the criteria for the control group (13 siblings and 8 patients in large total HV group; 14 siblings and 6 patients in large left HV group; 12 siblings and 6 patients in large right HV group).

In order to test the hypothesis that controls and siblings differed in their association between HV and cortisol stress-reactivity, the same model as described above was applied to cortisol (Incort) as the dependent variable. Additional confounders for all cortisol analyses were: time of cortisol sample, estrogen exposure and recent consumption of food or tobacco.

\section{Results}

\section{Descriptive analyses}

Groups were well matched on most demographic variables (Table 1). Patients smoked more cigarettes and cannabis than siblings and controls and had more lifetime hard drug use than siblings and controls, with no significant differences between the latter two groups. Eighteen patients received AP (atypical: $\mathrm{n}=2$; typical: $\mathrm{n}=16$ ). Furthermore, three patients used an antidepressant and one patient used a benzodiazepine. Two controls used an antidepressant, and one control used a benzodiazepine. Patients reported higher total, positive, and negative PANSS scores than controls and siblings, with no differences between the latter two groups (Table1). The mean illness duration in the patient group was 6.15 years $(\mathrm{SD}=3.45)$.

\section{Group differences in hippocampal volume, cortisol and stress reactivity}

There was a significant association between group and HV. Patients $(B=-247.6,95 \% \mathrm{Cl}-415.1,-80.01$; $p=0.01)$ and siblings $(B=-148.6,95 \% \mathrm{Cl}-292.66,-4.47 ; p=0.04)$ had smaller HV than controls. Patients and siblings did not differ ( $B=99.0,95 \% \mathrm{Cl}-57.63,255.61 ; p=0.22)$.

Siblings had significantly higher cortisol levels than controls $(B=0.28,95 \% \mathrm{Cl} 0.11,0.45 ; p=0.001)$. However, no significant association between cortisol levels and total $\mathrm{HV}(\mathrm{B}=-186.2,95 \% \mathrm{Cl}-398.16$, 25.76; $p=0.085)$, left $\mathrm{HV}(B=-157.1,95 \% \mathrm{Cl}-393.72,79.62 ; \mathrm{p}=0.19)$ or right $\mathrm{HV}(\mathrm{B}=-215.4,95 \% \mathrm{Cl}$ - 
452.63, 21.94; $p=0.075$ ) was found in the combined group of siblings and controls and no significant interaction between cortisol and group (siblings and controls) was found for total HV $\left(\chi^{2}(1)=0.00 ; p=\right.$ 0.99), left HV $\left(\chi^{2}(1)=0.03 ; p=0.85\right)$ or right $H V\left(\chi^{2}(1)=0.03 ; p=0.86\right)$.

There was no significant group $x$ event stress interaction in the model of NA $\left(\chi^{2}(2)=4.54, p=0.10\right)$ or in the model of cortisol $\left(\chi^{2}(1)=0.03 ; p=0.87\right)$.

\section{Emotional stress reactivity contingent on hippocampal volume and genetic risk}

A significant group $x$ event stress $x$ total HV interaction was found in the model of NA $\left(\chi^{2}(2)=6.54\right.$, $p=0.04)$. Differentiation by hemisphere revealed that the interaction with left $\operatorname{HV}\left(\chi^{2}(2)=7.90, p=0.02\right)$ and right $H V\left(\chi^{2}(2)=6.76, p=0.03\right)$ was significant. Thus, the association between total, left and right HV and stress reactivity differed between groups (Figure 1). Stratified analyses revealed increased emotional stress reactivity in controls with large HV in comparison to controls with small HV, being only significant for total HV (total: $\chi^{2}(1)=3.93, p=0.048$; left $\left(\chi^{2}(1)=3.13, p=0.077\right.$; right $\left(\chi^{2}(1)=1.95, p=0.16\right)$. In siblings, large HV was consistently associated with significantly less stress reactivity than small $\mathrm{HV}$ (total: $\chi^{2}(1)=6.89, p=0.009$; left: $\chi^{2}(1)=6.20, p=0.013$; right: $\chi^{2}(1)=5.73, p=0.017$ ). Patients with small left HV were significantly more stress reactive than patients with large left $H V$, while no difference in stress reactivity was found for total and right $\mathrm{HV}$ (total: $\chi^{2}(1)=1.20, p=0.27$; left: $\chi^{2}(1)=18.53, p<0.0001$; right: $\left.\chi^{2}(1)=0.06, p=0.81\right)$.

\section{AP use, antidepressant medication and depression}

Lifetime AP use did not predict HV in the patient group (total: $B=0.42,95 \% \mathrm{Cl}-1.91,2.75 ; \mathrm{p}=0.70$; left: $B=1.69,95 \% \mathrm{Cl}-0.83,4.21 ; p=0.17$; right: $B=-0.85,95 \% \mathrm{Cl}-3.18,1.48 ; p=0.45)$. Moreover, the results remained the same when $A P$ use, antidepressant use or history of depression were entered as additional predictor to the analyses.

\section{Cortisol stress reactivity contingent on hippocampal volume and genetic risk}

There was a significant group $x$ event stress $x$ total HV interaction in the model of cortisol $\left(\chi^{2}(1)=5.74\right.$, $\mathrm{p}=0.017)$. Differentiation by hemisphere revealed that the interaction was significant for left $H V\left(\chi^{2}\right.$ $(1)=4.89, p=0.027)$ and right $\mathrm{HV}\left(\chi^{2}(1)=3.89, p=0.049\right)$, suggesting that the association between total, left and right $\mathrm{HV}$ and cortisol reactivity to stress differed between the control and sibling group (Figure 2). For the control group, stratified analyses revealed no significant differences in cortisol response to daily stress between the hippocampal groups (total: $\chi^{2}(1)=0.19, p=0.66$; left $\left(\chi^{2}(1)=0.71, p=0.40\right.$; right $\left(\chi^{2}(1)=0.00, p=0.95\right)$. In the sibling group, however, large HV compared to small HV was associated with 
decreased cortisol responses to stress for total and left hippocampal groups (total: $\chi^{2}(1)=7.13, p=0.007$; left $\left(\chi^{2}(1)=7.38, p=0.007\right.$; right $\left(\chi^{2}(1)=0.89, p=0.35\right)$.

\section{Discussion}

To our knowledge, this is the first study to examine the association between stress sensitivity, indicators of HPA axis activity, and HV in a sample with different levels of genetic risk for psychosis. Results show that the immediate effect of daily stress on negative affect and cortisol was not only conditional on HV, but also on genetic risk for psychosis. Patients with a small left HV reported increased emotional stress reactivity compared to patients with large left HV. In line with the results in patients, siblings with small $\mathrm{HV}$ demonstrated increased emotional and cortisol reactivity to stress compared to those with large HV. In contrast, controls with a large total HV were more emotionally stress reactive than controls with small total HV, although this was not the case for cortisol reactivity.

\section{Hippocampal Volume and overall diurnal cortisol}

In line with a substantial amount of (meta-analytic) evidence [3, 4], HV in the patient group was smaller than that of control participants. Similarly, decreased HV in the siblings of patients with a psychotic disorder was found, which corresponds with findings from a recent meta-analysis [5, 6], as does the finding of absence of differences in HV between patients and their non-psychotic relatives [41].

There was no association between overall diurnal cortisol levels and $\mathrm{HV}$, which is in line with a previous study that found no association between HV and cortisol in first episode psychosis [42]. Another study, however, that differentiated between left and right $\mathrm{HV}$, found that baseline cortisol levels were associated with smaller left HV in first-episode psychosis, but not in controls [43]. Although we investigated left and right HV separately, no association between HV and cortisol level was apparent in siblings and controls, combined or in interaction. Nevertheless, there was a non-significant trend in the direction of a negative association between HV and diurnal cortisol levels, which is in line with the result of Mondelli and colleagues in first-episode psychosis [20].

\section{Stress reactivity and Hippocampal Volume in patients with psychotic disorder}

In patients with a psychotic disorder, smaller left HV was associated with increased emotional stress reactivity, while larger left HV was associated with a diminished emotional response to stress. Thus, patients with a psychotic disorder were not only more likely to have a small hippocampus, but those with a decreased left $\mathrm{HV}$ were also more likely to experience augmented stress reactivity. These findings 
are in line with earlier evidence that the hippocampus and in particular the left hippocampus [20] might have an inhibitory role on the human stress response, extending findings to the realm of daily life. Right HV, however, did not explain differences in stress reactivity.

We found differences in stress reactivity within the group of patients with psychotic disorder that could be traced to differences in left HV, i.e., patients with smaller left HV were most responsive to the environment. This finding underscores the notion that there might be different pathways to psychotic disorder, the stress related pathway being one of them [44, 45]. For example, patients with smaller HV may have experienced childhood trauma, impacting on cumulative glucocorticoid exposure and HV. The HV in turn, as shown in the current study, influences stress-reactivity.

It is important to note that the direction of the association between $\mathrm{HV}$ and stress reactivity is unresolved. The question of whether heightened cortisol reactivity may ultimately cause hippocampal volume changes or whether alterations in $\mathrm{HV}$, possibly through decreased inhibitory functioning of the hippocampus, are responsible for increased stress reactivity, should be answered in longitudinal studies.

\section{Stress reactivity and Hippocampal Volume in subjects at increased genetic risk for psychotic disorder}

Similar to the findings in patients, siblings with smaller HV exhibited increased emotional and cortisol stress reactivity, while larger HV in the siblings was associated with decreased emotional and cortisol responses to stress. Buchanan and colleagues (2009) suggest that the hippocampus may be a crucial element of a network involved in producing an integrated response to psychosocial stress (indexed by behavior and HPA activity). We found a decreased emotional and cortisol response to stress in siblings with larger HV and in patients a large left HV was associated with reduced emotional response to stress, as well. It may be that these represent markers for reduced integration of the stress response in those at increased risk for psychosis. In other words, a decreased emotional response and decreased cortisol concentrations in the siblings with larger HV, possibly reflecting suboptimal HPA axis functioning, may be a sign of a suboptimal response to psychosocial stress, resulting in increased liability for psychosis. Another possibility is that the blunted cortisol stress response in siblings with larger HV represents a protective factor against illness expression, given increased background vulnerability.

\section{Stress reactivity and Hippocampal Volume in healthy controls}

In controls, we found the reverse pattern, with smaller total HV associated with reduced emotional stress reactivity, and larger total HV associated with increased emotional stress reactivity. Cortisol reactivity to small daily hassles, however, did not differ as a function of $\mathrm{HV}$ in the control group. 
These findings contradict another study in healthy adults that report evidence for an association between stress level and smaller anterior HV. However, the stress measure used comprised retrospective summary information. In contrast, the current study measures emotional stress reactivity in daily life [46]. Our findings at the emotional level do correspond with a study by Pruessner and colleagues (2007), who found that larger HV in healthy young participants was associated with increased cortisol response to awakening (CAR) and to an experimental stressor [19]. Pruessner and co-workers (2007) speculated that a larger hippocampus may require increased cortisol concentrations for optimal functioning. However, we found no association between HV and cortisol stress reactivity in the control group. Differences in cortisol measures between the current study and the study by Pruessner and colleagues may constitute one explanation for the discrepant findings. Cortisol responses to laboratory stress and to awakening may affect different aspects of HPA axis reactivity than the reactivity to everyday hassles, which are likely more subtle stimuli. Nevertheless, the control participants with larger HV reported elevated emotional stress reactivity to naturally occurring stressors compared to those with smaller HV, which corresponds to the cortisol reactivity findings reported by Pruessner and colleagues (2007).

\section{Strength and weaknesses}

This study has several limitations. First, use of ESM booklets instead of electronic devices means that the exact timing of participants' self- reports and saliva samples cannot be firmly established [47]. However, results of a study comparing self-reported and electronically monitored saliva collection times, with the same intensive, semi-random time-sampling protocol used in the current study, indicated that saliva was generally collected very close to the prescribed time and that self-reported collection times corresponded well with the electronic time-stamps [48]. Another comparative study concluded that paper and electronic diaries yield similar results [49]. Second, no saliva samples were taken at the time of awakening, so that the current dataset does not allow examination of the CAR, a measure of HPA axis activity that appears to be blunted in first-episode psychosis [14] and may be associated with HV [19].

The current study also had some specific strengths. In particular, the repeated sampling of salivary cortisol over 6 days takes into account the well-known but often ignored unreliability of cortisol measures obtained at infrequent intervals [50]. Multiple cortisol measures per person were complemented by a relatively large number of participants. Use of multilevel modeling allowed assessment of withinperson associations between cortisol and subjective experience in real time and real-life contexts, as moderated by HV. Although cortisol measures were within the normal range, intensive sampling revealed different patterns of HPA activity with different HVs. Moreover, we combined measures of emotional and cortisol stress sensitivity with HV size, allowing a more comprehensive examination of HPA axis functioning. 
Disclosure/Conflicts of interest Jim van Os is or has been an unrestricted research grant holder with, or has received financial compensation as an independent symposium speaker from, Eli Lilly, BMS, Lundbeck, Organon, Janssen-Cilag, GlaxoSmithKline, AstraZeneca, Pfizer, and Servier. Machteld Marcelis has received financial compensation as an independent symposium speaker from Eli Lilly and Janssen-Cilag. Inez Myin-Germeys was supported by a 2006 NARSAD Young Investigator award and by the Dutch Medical Council (VENI and VIDI grant). She also received financial compensation as an independent symposium speaker from Eli Lilly, BMS, and Janssen-Cilag. Petra Habets, Dina Collip, Ed Gronenschild, Tineke Lataster, Mariëlle Lardinois and Nancy Nicolson reported no biomedical financial interests or potential conflicts of interest.

Acknowledgments This work was supported by a 2006 NARSAD Young Investigator Award and by the Dutch Medical Research Council (VENI and VIDI grants) to Dr. Inez Myin-Germeys. This work was sponsored by the Dutch organization for scientific research NWO (G.R.O.U.P.) and the European Community's Seventh Framework Programme under grant agreement No. HEALTH-F2-2009-241909 (EU-GEI consortium). The authors wish to thank the research assistants, Truda Driesen, Inge Crolla and Frieda Goethem for their work on this project, as well as the G.R.O.U.P. investigators*.

* René S. Kahn, Don H. Linszen, Jim van Os, Durk Wiersma; Richard Bruggeman, Wiepke Cahn, Lieuwe de Haan, Lydia Krabbendam, Inez Myin-Germeys. 

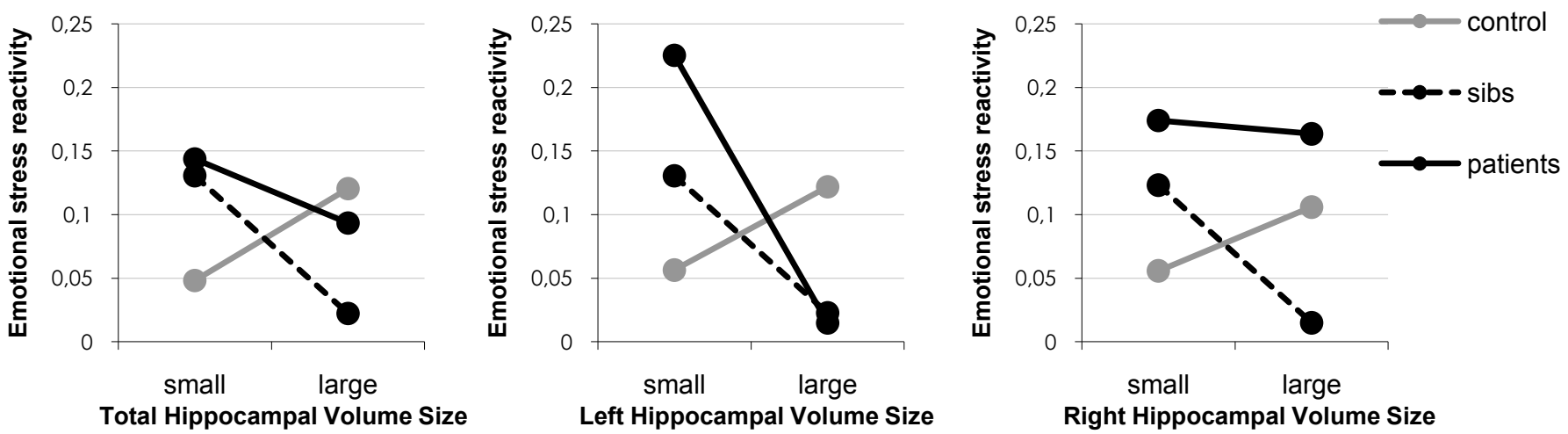

Figure 1. Emotional stress reactivity stratified by hippocampal volume, in control, sibling and patient groups: Multilevel estimates of the effects of daily events on negative affect. The models control for age, gender, intracranial volume and scan type. Effects are unstandardized regression coefficients.

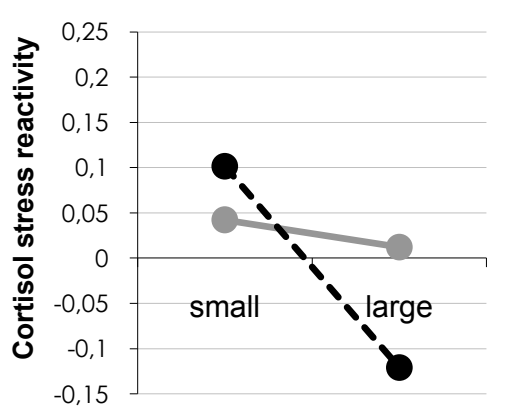

Total Hippocampal Volume Size

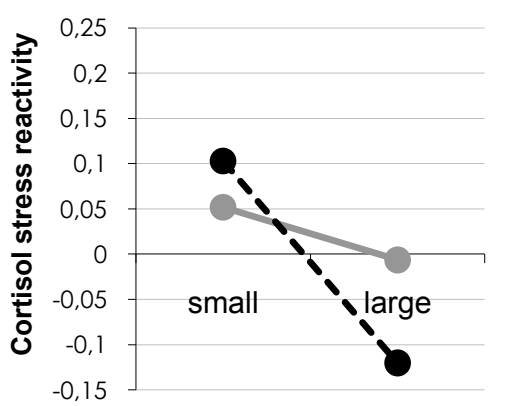

Left Hippocampal Volume Size

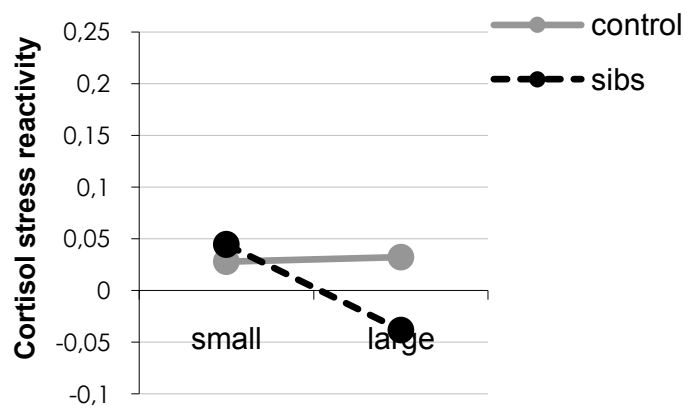

Right Hippocampal Volume Size

Figure 2. Cortisol stress reactivity stratified by hippocampal volume, in sibling and control groups: Multilevel estimates of the effects of daily events on cortisol. The dependent variable is log-transformed cortisol (Incort). The models control for time, age, gender, oral estrogen exposure, recent food intake, recent smoking and intracranial volume, and scantype. Effects are unstandardized regression coefficients. 
Table 1. Subject demographics

\begin{tabular}{|c|c|c|c|c|}
\hline & $\begin{array}{l}\text { Controls } \\
(n=32)\end{array}$ & $\begin{array}{l}\text { Siblings } \\
(n=37)\end{array}$ & $\begin{array}{l}\text { Patients } \\
(n=20)\end{array}$ & $\begin{array}{l}\text { Test statistic + } \\
\text { p-value }\end{array}$ \\
\hline Age, years: mean (SD) & $31.7(11.4)$ & $28.3(7.8)$ & $29.1(8.0)$ & $\mathrm{F}=1.38 P=0.24$ \\
\hline Gender (male:female) & $10: 22$ & $14: 23$ & 11:09 & $\chi 2=2.97 P=0.23$ \\
\hline Completed education \# & $5.8(1.8)$ & $5.3(2.2)$ & $4.7(1.8)$ & $\mathrm{F}=3.69 P=0.06$ \\
\hline \multicolumn{5}{|l|}{ PANSS Scores } \\
\hline Mean positive scale (SD) & $7.4(1.3)$ & $7.5(1.1)$ & $12.4(5.1)$ & $\mathrm{F}=28.89 P=0.00$ \\
\hline Mean negative scale (SD) & $8.0(0.2)$ & $8.2(1.0)$ & $10.8(3.3)$ & $\mathrm{F}=25.04 P=0.00$ \\
\hline Mean disorganization scale (SD) & $10.2(0.4)$ & $10.2(0.5)$ & $31.4(4.1)$ & $\mathrm{F}=23.55 P=0.00$ \\
\hline Mean excitement scale (SD) & $8.3(0.6)$ & $8.4(1.1)$ & $9.9(2.1)$ & $\mathrm{F}=14.11 P=0.00$ \\
\hline Mean emotional distress scale (SD) & $9.5(2.5)$ & $9.9(2.3)$ & $14.2(5.0)$ & $\mathrm{F}=20.57 P=0.00$ \\
\hline Mean alcohol use present state (SD) & $6.1(8.7)$ & $7.6(9.2)$ & $4.9(6.6)$ & $\mathrm{F}=0.11 P=0.74$ \\
\hline Mean cigarettes use present state (SD) & $1.1(3.9)$ & $1.8(4.6)$ & $12.1(11.9)$ & $\mathrm{F}=23.50 P=0.00$ \\
\hline \multicolumn{5}{|l|}{ Cannabis use, frequency } \\
\hline Mean cannabis use last 12 months (SD) & $1.6(8.8)$ & $3.4(12.6)$ & $34.3(99.8)$ & $\mathrm{F}=4.79 P=0.03$ \\
\hline Mean cannabis use lifetime (SD) & $16.8(34.4)$ & $19.6(35.7)$ & $54.2(48.0)$ & $\mathrm{F}=0.00 P=0.00$ \\
\hline \multicolumn{5}{|l|}{ Hard drug use, frequency } \\
\hline Mean last 12 months hard drug use (SD) & $1.6(9.2)$ & $0.0(0.0)$ & $5.0(18.2)$ & $\mathrm{F}=0.89 P=0.4$ \\
\hline Mean lifetime hard drug use (SD & $3.4(13.0)$ & $5.1(15.4)$ & $40.5(73.05)$ & $\mathrm{F}=8.66 P=0.01$ \\
\hline \multicolumn{5}{|l|}{ Antipsychotics } \\
\hline Type antipsychotic (typical:atypical) & & & 2:16 & \\
\hline Mean haldolequivalent present state(SD) & & & $2.4(1.9)$ & \\
\hline \multicolumn{2}{|c|}{ Mean total antipsychotic use in haldolequivalent (SD) } & & $48.2(47.1)$ & \\
\hline Mean lifetime estorgen exposure (SD) & $17589(31700)$ & $18474(29410)$ & $672(2013)$ & $\mathrm{F}=2.84 P=0.10$ \\
\hline Scantype (MDEFT:ADNI) & 23:09 & $26: 11$ & $16: 04$ & $\chi 2=0.7 P=0.72$ \\
\hline Hippocampal volume, mm3 (SD) & 3669 (340) & 3618 (384) & 3491 (319) & $F=10.25 P=0.003$ \\
\hline Cortisol, nmol/L (SD) & $2.65(0.89)$ & $3.63(1.3)$ & & $F=11.95 P=0.001$ \\
\hline ESM observations, No. & $44.19(10.7)$ & $40.68(9.16)$ & $42.05(9.96)$ & $\mathrm{F}=0.88 P=0.35$ \\
\hline
\end{tabular}

Abbreviations: PANSS, Positive and Negative Syndrome Scale

$\mathrm{F} / \mathrm{Chi}^{2}$ and $\mathrm{P}$-values refer to between-group differences

\# Educational level was defined as highest accomplished level of education. 


\section{References}

1. Geuze E, Vermetten E, and Bremner JD (2005): MR-based in vivo hippocampal volumetrics: 2. Findings in neuropsychiatric disorders. Mol Psychiatry 10:160-84.

2. Steen RG, Mull C, McClure R, Hamer RM, and Lieberman JA (2006): Brain volume in first-episode schizophrenia: systematic review and meta-analysis of magnetic resonance imaging studies. Br J Psychiatry 188:510-8.

3. Wright IC, Rabe-Hesketh S, Woodruff PW, David AS, Murray RM, and Bullmore ET (2000): Meta-analysis of regional brain volumes in schizophrenia. Am J Psychiatry 157:16-25.

4. Vita A, De Peri L, Silenzi C, and Dieci M (2006): Brain morphology in first-episode schizophrenia: a meta-analysis of quantitative magnetic resonance imaging studies. Schizophr Res 82:75-88.

5. Lawrie SM, Mclntosh AM, Hall J, Owens DG, and Johnstone EC (2008): Brain structure and function changes during the development of schizophrenia: the evidence from studies of subjects at increased genetic risk. Schizophr Bull 34:330-40.

6. Boos H, Aleman A, Cahn W, Pol HH, and Kahn RS, (2007): Brain volumes in relatives of patients with schizophrenia: a meta-analysis. Am Med Assoc. p. 297.

7. Corcoran C, Walker E, Huot R, Mittal V, Tessner K, Kestler L, et al. (2003): The stress cascade and schizophrenia: etiology and onset. Schizophr Bull 29:671-92.

8. O'Brien JT, (1997): The'glucocorticoid cascade'hypothesis in man: prolonged stress may cause permanent brain damage. Maney. p. 199-201.

9. Sapolsky RM, (2000): Glucocorticoids and hippocampal atrophy in neuropsychiatric disorders. Am Med Assoc. p. 925.

10. Buchanan TW, Tranel D, and Kirschbaum C (2009): Hippocampal damage abolishes the cortisol response to psychosocial stress in humans. Horm Behav 56:44-50.

11. Jacobson L and Sapolsky R (1991): The role of the hippocampus in feedback regulation of the hypothalamicpituitary-adrenocortical axis. Endocr Rev 12:118-34.

12. Lupien SJ, de Leon M, De Santi S, Convit A, Tarshish C, Nair NPV, et al., (1998): Cortisol levels during human aging predict hippocampal atrophy and memory deficits. Nature Publishing Group. p. 69-73.

13. Knoops AJG, Gerritsen L, van der Graaf Y, Mali W, and Geerlings MI, (2011): Basal Hypothalamic Pituitary Adrenal Axis Activity and Hippocampal Volumes: The SMART-Medea Study. Elsevier.

14. Mondelli V, Dazzan P, Hepgul N, Di Forti M, Aas M, D’Albenzio A, et al. (2010): Abnormal cortisol levels during the day and cortisol awakening response in first-episode psychosis: the role of stress and of antipsychotic treatment. Schizophr Res 116:234-42.

15. Pruessner JC, Dedovic K, Pruessner M, Lord C, Buss C, Collins L, et al. (2008): Stress regulation in the central nervous system: evidence from structural and functional neuroimaging studies in human populations - 2008 Curt Richter Award Winner. Psychoneuroendocrinology 35:179-91.

16. Tessner KD, Walker EF, Dhruv SH, Hochman K, and Hamann S, (2007): The relation of cortisol levels with hippocampus volumes under baseline and challenge conditions. Elsevier. p. 70-78.

17. Myin-Germeys I, Delespaul P, and van Os J (2005): Behavioural sensitization to daily life stress in psychosis. Psychol Med 35:733-41.

18. Myin-Germeys I, van Os J, Schwartz JE, Stone AA, and Delespaul PA (2001): Emotional reactivity to daily life stress in psychosis. Arch Gen Psychiatry 58:1137-44.

19. Pruessner M, Pruessner JC, Hellhammer DH, Bruce Pike G, and Lupien SJ (2007): The associations among hippocampal volume, cortisol reactivity, and memory performance in healthy young men. Psychiatry Res 155:1-10.

20. G.R.O.U.P. (2011): Evidence that Familial Liability for Psychosis is Expressed as Differential Sensitivity to Cannabis: an Analysis of Patient-Sibling and Sibling-Control Pairs. Arch Gen Psychiatry in press. 
21. Maxwell ME (1992): Family Interview for Genetic Studies (FIGS): Manual For FIGS. 1992, Clinical Neurogenetics Branch, Intramural Research Program, National Institute of Mental Health, Bethesda, MD.

22. APA (2000): Diagnostic and statistical manual of mental disorders. 4th ed. 2000, Washington, DC: American Psychiatric Association.

23. Andreasen NC, Flaum M, and Arndt S (1992): The Comprehensive Assessment of Symptoms and History (CASH). An instrument for assessing diagnosis and psychopathology. Arch Gen Psychiatry 49:615-23.

24. Kay SR, Fiszbein A, and Opler LA (1987): The positive and negative syndrome scale (PANSS) for schizophrenia. Schizophr Bull 13:261-76.

25. Cahn W, Pol HE, Lems EB, van Haren NE, Schnack HG, van der Linden JA, et al. (2002): Brain volume changes in firstepisode schizophrenia: a 1-year follow-up study. Arch Gen Psychiatry 59:1002-10.

26. WHO, (1990): Composite International Diagnostic Interview (CIDI). World Health Organization: Geneva.

27. Csikszentmihalyi M and Larson R (1987): Validity and reliability of the Experience-Sampling Method. J Nerv Ment Dis 175:526-36.

28. Myin-Germeys I, Oorschot M, Collip D, Lataster J, Delespaul P, and van Os J (2009): Experience sampling research in psychopathology: opening the black box of daily life. Psychol Med 39:1533-47.

29. Delespaul P, deVries M, and van Os J (2002): Determinants of occurrence and recovery from hallucinations in daily life. Soc Psychiatry Psychiatr Epidemiol 37:97-104.

30. Jacobs N, Myin-Germeys I, Derom C, Delespaul P, van Os J, and Nicolson NA (2007): A momentary assessment study of the relationship between affective and adrenocortical stress responses in daily life. Biol Psychol 74:60-6.

31. Sulon J, Demey-Ponsart L, Beauduin P, and Sodoyez JC (1978): Radioimmunoassay of corticosterone, cortisol and cortisone: their application to human cord and maternal plasma. J Steroid Biochem 9:671-6.

32. Dale AM, Fischl B, and Sereno MI (1999): Cortical surface-based analysis. I. Segmentation and surface reconstruction. Neuroimage 9:179-94.

33. Fischl B and Dale AM (2000): Measuring the thickness of the human cerebral cortex from magnetic resonance images. Proc Natl Acad Sci U S A 97:11050-5.

34. Fischl B, Salat DH, Busa E, Albert M, Dieterich M, Haselgrove C, et al. (2002): Whole brain segmentation: automated labeling of neuroanatomical structures in the human brain. Neuron 33:341-55.

35. Fischl B, Sereno MI, and Dale AM (1999): Cortical surface-based analysis. II: Inflation, flattening, and a surfacebased coordinate system. Neuroimage 9:195-207.

36. Han X, Jovicich J, Salat D, van der Kouwe A, Quinn B, Czanner S, et al. (2006): Reliability of MRI-derived measurements of human cerebral cortical thickness: the effects of field strength, scanner upgrade and manufacturer. Neuroimage 32:180-94.

37. Jovicich J, Czanner S, Greve D, Haley E, van der Kouwe A, Gollub R, et al. (2006): Reliability in multi-site structural MRI studies: effects of gradient non-linearity correction on phantom and human data. Neuroimage 30:436-43.

38. Segonne F, Dale AM, Busa E, Glessner M, Salat D, Hahn HK, et al. (2004): A hybrid approach to the skull stripping problem in MRI. Neuroimage 22:1060-75.

39. StataCorp (2009): STATA/SE statistical software, release 11. 2009, Texas: College Station.

40. Meador-Woodruff JH and Greden JF, (1988): Effects of psychotropic medications on hypothalamic-pituitary-adrenal regulation. p. 225.

41. Seidman LJ, Faraone SV, Goldstein JM, Kremen WS, Horton NJ, Makris N, et al. (2002): Left hippocampal volume as a vulnerability indicator for schizophrenia: a magnetic resonance imaging morphometric study of nonpsychotic first-degree relatives. Vol. 59. 2002: Am Med Assoc. 839.

42. Gunduz-Bruce H, Szeszko PR, Gueorguieva R, Ashtari M, Robinson DG, Kane JM, et al., (2007): Cortisol levels in relation to hippocampal sub-regions in subjects with first episode schizophrenia. Elsevier. p. 281-287. 
43. Mondelli V, Pariante CM, Navari S, Aas M, D’Albenzio A, Di Forti M, et al. (2010): Higher cortisol levels are associated with smaller left hippocampal volume in first-episode psychosis. Schizophr Res 119:75-78.

44. Lataster T, Collip D, Lardinois M, van Os J, and Myin-Germeys I (2010): Evidence for a familial correlation between increased reactivity to stress and positive psychotic symptoms. Acta Psychiatr Scand 122:395-404.

45. Myin-Germeys I and van Os J (2007): Stress-reactivity in psychosis: evidence for an affective pathway to psychosis. Clin Psychol Rev 27:409-24.

46. Szeszko PR, Betensky JD, Mentschel C, Gunduz-Bruce H, Lencz T, Ashtari M, et al. (2006): Increased stress and smaller anterior hippocampal volume. Neuroreport 17:1825-8.

47. Stone AA, Shiffman S, Schwartz JE, Broderick JE, and Hufford MR (2002): Patient non-compliance with paper diaries. BMJ 324:1193-4.

48. Jacobs N, Nicolson NA, Derom C, Delespaul P, van Os J, and Myin-Germeys I (2005): Electronic monitoring of salivary cortisol sampling compliance in daily life. Life Sciences76:2431-43.

49. Green AS, Rafaeli E, Bolger N, Shrout PE, and Reis HT (2006): Paper or plastic? Data equivalence in paper and electronic diaries. Psychol Methods 11:87-105.

50. Hruschka DJ, Kohrt BA, and Worthman CM (2005): Estimating between- and within-individual variation in cortisol levels using multilevel models. Psychoneuroendocrinology 30:698-714. 
Chapter 6 
${ }^{1}$ Department of Psychiatry and Neuropsychology, South Limburg Mental Health Research and Teaching Network, EURON, School for Mental Health and NeuroScience MHeNS Maastricht University, Maastricht, The Netherlands

${ }^{2}$ University Psychiatric Centre Catholic University Leuven, Kortenberg, Belgium

${ }^{3}$ Faculty of Psychology, Open University of the Netherlands, Heerlen, The Netherlands

${ }^{4}$ Division of Psychological Medicine, Institute of Psychiatry, London, UK

*contributed equally 


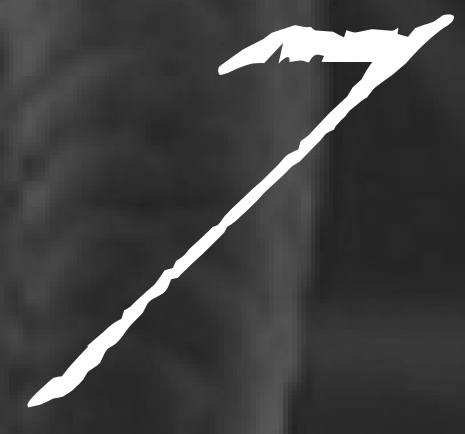

$$
\begin{gathered}
\text { COMTVali58Met-Stress } \\
\text { Interaction in Psychosis: } \\
\text { Role of Background Psychosis Risk }
\end{gathered}
$$

Dina Collip ${ }^{1}$, Ruud van Winkel ${ }^{1,2 *}$, Odette Peerbooms ${ }^{1 *}$, Tineke Lataster $^{1}$, Viviane Thewissen ${ }^{1,3}$, Marielle Lardinois ${ }^{1}$, Marjan Drukker ${ }^{1}$, Bart P.F. Rutten ${ }^{1}$, Jim Van Os ${ }^{1,4}$ \& Inez Myin-Germeys ${ }^{1}$

CNS Neuroscience \& Therapeutics 2010; Nov 14. doi: 10.1111/j.1755-5949.2010.00213.x. 


\section{Abstract}

\section{Background}

The interplay between the catechol-O-methyltransferase (COMT) Val158Met polymorphism and environmental stress may have aetiological relevance for psychosis, but differential effects have been reported in healthy control and patient groups, suggesting that COMT Val158Met interactions with stress may be conditional on background genetic risk for psychotic disorder.

\section{Methods}

Patients with a non-affective psychotic disorder $(n=86)$ and control participants $(n=109)$ were studied with the Experience Sampling Method (a structured diary technique) in order to assess stress, negative affect and momentary psychotic symptoms in the flow of daily life.

\section{Results}

Multilevel analyses revealed significant three-way interactions between group status (patient or control), COMT genotype and stress in the model of negative affect $\left(\chi^{2}(2)=13.26, p<0.01\right)$ as well as in the model of momentary psychotic symptoms $\left(\chi^{2}(2)=6.92, p<0.05\right)$. Exploration of the 3 -way interaction revealed that in patients, COMT genotype moderated the association between stress and negative affect $\left(\chi^{2}(4)=11.50, p<0.005\right)$, as well as the association between stress and momentary psychosis $\left(\chi^{2}(4)=12.79, p<0.005\right)$. Met/Met genotype patients showed significantly increased psychotic and affective reactivity to stress in comparison to the Val/Met and Val/Val genotypes. In contrast, healthy controls did not display large or significant COMT Val158Met X stress interactions.

\section{Conclusions}

Important differences exist in the effect of COMT Val158Met on stress reactivity, which may depend on background risk for psychotic disorder. Differential sensitivity to environmental stress occasioned by COMT Val158Met may be contingent on higher order interactions with genetic variation underlying psychotic disorder.

Key words: genes/environment/schizophrenia/psychological stress/experience sampling method/ psychotic disorders 


\section{Introduction}

There is evidence that interplay between genes and environment constitutes a sufficient cause for the development of psychotic disorder [1]. Studies that have examined molecular genetic variation associated with differential sensitivity to environmental pathogens remain rare, however, emphasizing the need for further investigation of gene-environment interactions and their neurobiological underpinnings [2].

Environmental stress exposure likely represents an important factor in the developmental trajectory towards psychosis [3]. One area of genetic variation that has been extensively explored as possible moderator of environmental influences in psychosis is the Val158Met functional polymorphism of the catechol-O-methyltransferase (COMT) gene. While the Val-allele carriers have been found to be more sensitive to the psychotogenic effects of cannabis [4, 5], results of some COMT-stress interaction studies point towards exaggerated sensitivity to stress in Met-carriers. In animal research, transgenic mice overexpressing human COMT-Val polymorphism showed a blunted stress response, while COMT knock-out, "Met-like" mice exhibited increased stress response [6]. Likewise, in an experimental human study, COMT Met158 homozygotes exhibited a markedly potentiated startle reflex in reaction to aversive stimuli compared with Val-carriers, suggesting increased emotional dysregulation in Metcarriers [7]. This heightened emotional reactivity in Met-carriers might also contribute to a higher risk for psychopathology in individuals exposed to stress [8]. The first study evaluating the effect of COMT Val158Met on stress-reactivity in daily life in patients with a psychotic disorder, in comparison with healthy controls, reported that COMT Val158Met moderated psychotic and emotional reactivity to stress in patients, the Met/Met genotype displaying greatest reactivity to stress [9]. Interestingly, this effect was not found in the healthy controls, suggesting that the COMT Val158Met interactions with stress may be contingent on background of genetic risk for psychotic disorder. Given the fact that the findings reported by van Winkel and colleagues (2008a) were based on a relatively small sample of patients and controls who were all daily cannabis users, replication of these findings in a larger sample of patients and healthy controls, with adequate control for cannabis use is necessary.

The experience sampling method is a structured diary technique that captures mental states and small stressors in the flow of daily life [10]. ESM allows for a prospective, repeated measure of proximal environmental stress and when combined with genetic information, offers an elegant way to test gene-environment interactions $[10,11]$. Thus, in an independent sample from that of van Winkel and colleagues (2008a) ESM was used to investigate how changes in emotional and psychotic experiences may vary with naturally occurring stressors and molecular genetic variation in COMT. Specifically, it was investigated (i) whether group (patient vs. control) moderated the association between (affective and psychotic) reactivity to stress and the COMT Val158Met polymorphism; and (ii) how, in case of a significant interaction, the COMT Val158Met polymorphism moderated affective and psychotic reactivity to daily stress within the two groups. 
We hypothesized that heightened affective and psychotic stress reactivity would be evident in patients with the Met/Met genotype, but not in the control sample.

\section{Methods}

\section{Sample}

The sample consisted of control subjects and patients diagnosed with a non-affective psychotic disorder. Prior to entering the study, all subjects were screened. Inclusion criteria were: (1) age 1865 years; (2) sufficient command of the Dutch language. Exclusion criteria were: (1) brain disease; (2) history of head injury with loss of consciousness. Exclusion criteria for control participants were (3) lifetime history of psychotic disorder or mood disorder and (4) family history of psychotic disorder. In selected representative geographical areas in the South of the Netherlands and the Dutch-speaking part of Belgium, patients were identified through representative clinicians working in regional psychotic disorder services, whose caseload was screened for inclusion criteria. Subsequently, a group of patients presenting consecutively at these services either as out-patients or in-patients were recruited for the study. Interview data and clinical record data assessing affective and psychotic symptoms were used in patients and controls to complete the Comprehensive Assessment of Symptoms and History [CASH; [12]] or the Operational Criteria Checklist for Psychotic Illness (OCCPI) yielding DSM-IV diagnoses through the OPCRIT computer program [13]. Controls were selected through a system of random mailings to addresses in the catchment areas of the cases. All participants were derived from two earlier studies [for more information on sample, see 14, 15]. The original sample comprised a total number of 316 participants (controls and patients with a non-affective psychotic disorder). Written informed consent, conforming to the local ethics committee's guidelines, was obtained from all participants.

\section{COMT Val158Met Genotyping}

DNA was collected either by buccal mucosa or by blood. Buccal cell samples were collected with sterile swabs (Omniswab, Whatman '). DNA was extracted using QIAamp DNA Mini Kits (Qiagen). DNA of the blood samples was isolated either manually according to the Promega protocol or with the Autogenflex3000.

A single SNP was genotyped using the following TaqMan ${ }^{\circ}$ SNP Genotyping assay (Applied Biosystems, Nieuwerkerk a/d IJssel, The Netherlands): rs4680 (assay ID C_25746809_50). The assay was run on a 7900HT Fast Real-Time PCR System (Applied Biosystems). 


\section{ESM}

The Experience Sampling Method (ESM) is a structured random time-sampling self-assessment technique to assess mental states and contexts in daily living environments. It is a valid and reliable way to study immediate effects of the environment, reducing biases in recall [10]. Participants received a pre-programmed digital wristwatch and ESM self-assessment forms collated in a booklet for each day. Ten times a day on 6 consecutive days, the watch emitted a signal at unpredictable moments between 7.30 a.m. and 10.30 p.m. After each "beep", subjects were asked to fill out the ESM self-assessment forms previously handed to them, collecting reports of thoughts, current context (activity, persons present and location), appraisals of the current situation, mood and psychotic experiences. All selfassessments were rated on 7-point Likert scales. Participants were instructed to complete their reports immediately after the beep, in order to minimize memory distortions, and to record the time at which they completed the form. During the sampling period, research staff contacted subjects by phone to assess whether they were complying with the instructions. Reports are assumed valid when subjects respond to the beep within 15 minutes. This was ascertained by comparing the actual beep time with the reported time of completion of the reports. All reports completed more than 15 minutes after the signal were excluded from the analyses. Participants were only included in the analyses, when they provided valid responses to at least one-third of the emitted beeps [16]. Previous studies have demonstrated the feasibility, validity, and reliability of ESM in general and patient populations [10, 17, 18].

\section{Measures}

Stress reactivity, as described in previous work $[9,19]$, was conceptualized as affective and psychotic reactivity to daily life events and minor disturbances in daily life. Measures of stress, mood and psychotic symptoms were derived from the experience sampling reports as described below.

Momentary stress. In accordance with previous work, stress was conceptualized as the subjectively appraised stressfulness of distinctive events (event stress) [20]. In order to measure event stress, the subject was asked to report, after each beep, the most important event that had happened between the current and the previous report. This event was subsequently rated on a bipolar Likert scale ($3=$ very unpleasant, $0=$ neutral, $3=$ very pleasant). The responses were recoded to allow high scores to reflect stress $(-3=$ very pleasant, $0=$ neutral, $3=$ very unpleasant).

ESM psychosis. Momentary psychosis was defined as the sum score of 6 ESM items I feel suspicious, I cannot get rid of my thoughts, I am afraid of loosing control, I feel unreal, and I hear voices, I see phenomena. All items were rated on 7-point Likert scales (ranging from not at all to very) (Cronbachs $\alpha=0.72$ ). 
ESM hallucinations. Momentary hallucinations were defined as the mean score of 2 ESM items I hear voices and I see phenomena. Both items were rated on 7-point Likert scales (ranging from not at all to very) (Cronbachs $\alpha=0.77$ ).

ESM delusions. Momentary delusions were defined as the mean score of 4 ESM items I feel suspicious, I cannot get rid of my thoughts, I am afraid of loosing control, and I feel unreal. All items were rated on 7-point Likert scales (ranging from not at all to very) (Cronbachs $\alpha=0.68$ ).

Negative affect. After each beep, participants were asked to answer questions regarding their mood, on 7-point Likert scales ( $1=$ not at all, $7=$ =very). In line with previous reports [19] ESM negative affect was assessed with 6 mood-related adjectives (down, guilty, insecure, lonely, anxious, angry/ irritated) and were reduced to one measure of the mean Negative Affect (NA) (Cronbach's $\alpha=0.83$ ).

\section{Cannabis measures}

We assessed use of cannabis using the L-section of the M-CIDI [21], which includes a variety of other substances. A variable for current cannabis use and cannabis use of the last 12 months was constructed. Drug use information was available for 144 participants.

\section{Statistical Analyses}

Multilevel linear regression analysis, which is ideally suited for analyses of clustered data [22], was used since ESM data have a hierarchical structure with repeated momentary measurements per subject. Analyses were carried out with the XTREGAR module in STATA/MP version 10.1 [23]. XTREGAR takes into account clustering of data at the level of the beep (moment of the day, level 1) within individuals (level 2), as well as clustering of observations directly following one another (i.e. autocorrelation).

Outcome variables included in the analyses were standardized by dividing the variables by the group standard deviation of this variable, yielding standardized effect sizes.

In order to test the hypothesis that COMT Val158Met genotype moderates psychotic and affective responses to daily life stress, and whether any moderation differs between patients and controls, a three-way interaction between COMT Val158Met genotype, group and event stress was tested with ESM psychosis (delusions, hallucinations) and ESM NA as the respective dependent variables. The independent variables were COMT Val158Met genotype ( $0=\mathrm{Val} / \mathrm{Val} ; 1=\mathrm{Val} / \mathrm{Met} ; 2=\mathrm{Met} / \mathrm{Met})$, event stress ( $-3=$ very pleasant, $0=$ neutral, $3=$ very unpleasant $)$ and group ( $0=$ controls; $1=$ patients). Main effects and interactions were assessed by Wald test. Stratified effects were calculated by applying and testing the appropriate linear combinations using the STATA LINCOM command. 


\section{Results}

\section{Sample}

Of the 316 participants (171 patients and 145 controls) who entered the study, 216 provided DNA (98 patients and 118 control participants). Genotyping failed in 13 participants. 2 control participants were excluded due to failure to complete the protocol (missing information regarding the time of the beep and completion of questionnaires). An additional 5 patients and 1 control were excluded from the analyses as they had an insufficient number of valid ESM observations $(<20)$.

The final sample for analysis thus comprised 195 participants (86 patients and 109 control participants). These participants had each completed an average of 44 valid ESM reports $(S D=9)$ (control group: 47 $(S D=8)$ and patients: $40(S D=9) ; \beta[S E]=-3.5$ [.61]; $p<.001)$. The patients were all diagnosed with a nonaffective psychotic disorder (41 with schizophrenia; 10 with schizoaffective disorder; 26 with psychotic disorder NOS; 1 with schizophreniform disorder; 3 with delusional disorder; 5 with a brief psychotic disorder). Patients with a diagnosis of schizoaffective disorder were retained in the sample since i) all included patients, including the schizoaffective patients, were not in an acute psychotic or affective episode, and ii) there is no evidence that affective disorders such as (current) major depressive disorder or bipolar disorder are associated with greater reactivity to stress compared to schizophrenia patients [24]. Additional information regarding number of valid reports and sociodemographic and clinical characteristics of the sample are depicted in Table 1.

\section{COMT Val158Met genotype}

The frequencies of the three COMT genotypes were $24.6 \%(n=48) \mathrm{Val} / \mathrm{Val}, 47.2 \%(n=92) \mathrm{Val} / \mathrm{Met}$ and $28.2 \%(n=55)$ Met/Met, and were in Hardy-Weinberg equilibrium $\left(\chi^{2}(1)=0.59, P=0.44\right)$. Genotype was not associated with age, sex, ESM psychosis, ESM hallucinations, ESM delusions, ESM stress or NA (Table 2). Also, COMT genotype did not predict current cannabis use or cannabis use in the past 12 months. Moreover, there was no main effect of group on COMT genotype $\left(\chi^{2}(d f=2)=2.75, P=0.25\right)$.

\section{Models for NA}

A significant main effect was found for event stress on NA ( $\beta[S E]=.09$ [.01]; $p<.001)$. Thus, independent of group or genotype, the more subjective stress participants reported, the more NA they experienced. Furthermore, multilevel analyses revealed a significant three-way interaction between COMT Val158Met, event stress and group in the model of $N A\left(\chi^{2}(d f=2)=13.26, p<0.01\right)$, suggesting that the association between stress and NA is moderated by COMT genotype, dependent on group status (patient or control). 
Further exploration of the group X COMT Val158Met X ESM stress interaction revealed a strong twoway interaction between COMT Val158Met genotype and ESM stress in the model of NA in patients $\left(\chi^{2}(d f=2)=11.50, p<0.005\right)$, but not in controls $\left(\chi^{2}(d f=2)=3.38, p=0.19\right)$. Met/Met genotype patients reported a larger increase in NA after event stress than did Val/Met or Val/Val genotypes (Figure 1; Table 3).

\section{Models for ESM psychosis}

No main effect was found for event stress on ESM psychosis ( $\beta[S E]=.01$ [.01]; $p=.26)$. However, a significant three-way interaction between COMT Val158Met genotype, event stress and group in the model of ESM psychosis $\left(\chi^{2}(d f=2)=6.92, p<0.05\right)$ suggested that the association between event stress and psychosis is not only moderated by COMT Val158Met genotype but is also dependent on group status.

Again, a significant two-way interaction between COMT Val158Met genotype and event stress in the model of ESM psychosis was apparent in the patient group $\left(\chi^{2}(\mathrm{df}=2)=12.79, p<0.005\right)$, but not in the control group $\left(\chi^{2}(d f=2)=0.99, p=0.61\right)$. Met/Met genotype patients showed significantly increased psychotic reactivity to stress in comparison to the Val/Met and Val/Val genotypes (Table 3).

Comparable results were found for ESM delusions $\left(\chi^{2}(\mathrm{df}=2)=13.63, p<0.005\right)$ (see Figure 2 for effect sizes of stratified effects). For ESM hallucinations, neither a significant three-way nor a significant twoway interaction was found.

\section{Sensitivity Analysis in non-cannabis users}

Additional analyses were carried out, investigating whether cannabis use impacted the results. All analyses were repeated with exclusion of participants who were current cannabis users or used cannabis in the last 12 months. Apart from some small effect size alterations, results were comparable.

The three-way interaction between COMTVal158Met genotype, event stress and group in the model of NA $\left(\chi^{2}(d f=2)=15.43, p<0.001\right)$, ESM psychosis $\left(\chi^{2}(d f=2)=15.06, p<0.001\right)$ and ESM delusions $\left(\chi^{2}(d f=2)=20.6\right.$, $p<0.001)$ remained significant. Similarly, the two-way interaction between COMT Val158Met genotype and ESM stress in the model of NA, ESM psychosis and ESM delusions was significant in patients $\left(\chi^{2}(\mathrm{df}=2)=15.50, p<0.001 ; \chi^{2}(\mathrm{df}=2)=23.67, \mathrm{p}<0.001 ; \chi^{2}(\mathrm{df}=2)=31.6, \mathrm{p}<0.001\right.$, respectively), but not in controls $\left(\chi^{2}(\mathrm{df}=2)=3.04, p=0.22 ; \chi^{2}(\mathrm{df}=2)=1.25, p=0.53 ; \chi^{2}(\mathrm{df}=2)=0.39, p=0.83\right.$, respectively). Met/Met genotype patients reported a larger increase in NA, ESM psychosis and ESM delusions after event stress than did Val/Met or Val/Val genotypes. Again, for ESM hallucinations, neither a significant three-way nor a significant two-way interaction was found. 


\section{Models of mediated moderation}

Since NA and ESM psychosis were significantly associated ( $\beta[S E]=.40$ [.01]; $p<0.001)$, models of mediated moderation were also examined. There was no evidence that ESM psychosis was a mediator of the interactive effects of ESM stress and COMT genotype in the model of NA (three-way interaction $\left.\chi^{2}(d f=2)=8.2, p<0.05\right)$. The COMT X ESM stress interaction was no longer significant in the model of ESM psychosis after covarying for $N A$ (three-way interaction $\chi^{2}(d f=2)=2.62, p=0.27$ ), however, while the interaction did remain significant in the model of ESM delusions $\left(\chi^{2}(d f=2)=6.31, p<0.05\right)$ although with lower effect sizes, suggesting partial mediation by NA in models of psychosis.

\section{Discussion}

The present study investigated the moderating effect of COMT Val158Met on the association between real-life stress and psychosis as well as on the association between real-life stress and negative affect. It was shown that the immediate effect of daily stress on psychosis and negative affect is not only conditional on COMT Val158Met genotype, but also on group, supporting the hypothesis that this specific instance of gene-environment interaction may be contingent on higher-order interactions with other background genetic risk variants associated with psychotic disorder. COMT Val158Met genotype contributed to differential sensitivity to environmental stress only in the patient group. More specifically, Met/Met genotype patients reported a larger increase in NA and in momentary psychosis (particularly delusions) in reaction to stress than patients with the Val/Met or Val/Val genotype. In addition, no main effects of COMT Val158Met on momentary psychosis or NA were found.

\section{COMT Val158Met and psychosis}

The absence of COMT main effects are in support of earlier case-control studies and meta-analyses examining its association with schizophrenia $[25,26]$, suggesting that the harmful or beneficial effects of COMT Val158Met may be conditional on type, timing and level of environmental exposure, consistent with pleiotropic behavioral effects of COMT genetic variation [27]. Thus, the present results are in support of a recent study by van Winkel and colleagues (2008a), reporting that Met/Met genotypes in the patient group were most stress reactive, showing more psychotic symptoms and negative affect in reaction to stress, while no such effect was found in the control sample. However, their study sample consisted exclusively of cannabis users. The present study confirmed these initial findings in a much larger sample, with adequate control for the possible confounding effects of cannabis use. Two earlier studies examining psychotic stress response as a function of COMT Val158Met in non-patient healthy 
controls are in agreement with the current finding that psychotic stress response in the control group was not contingent on Met/Met genotype [28, 29]. However, these two studies also differed in relation to the current results, in that an amplified psychotic stress-response in healthy subjects did show association with Val/Val genotype relative to Met carriers. These studies, however, either used a stress exposure distal to the examined phenotype (army induction) or used a fairly limited assessment of psychotic-like experiences.

\section{COMT Val158Met, emotional reactivity and background risk for psychotic disorder}

The finding that COMT Val158Met plays a role in emotional reactivity to stress corresponds with other studies reporting increased sensitivity to stress, anxiety and pain in Met allele carriers [30-32]. Furthermore, a meta-analysis of neuro-imaging studies was in agreement with these findings, showing an increased prefrontal activation in Met-carriers in emotional paradigms [27]. Most of these studies, however, were conducted in general population samples, while the current results only showed increased reactivity for Met/Met genotype patients but not Met/Met genotype controls. A lack of statistical power, given lower variability associated with behavioral measurements of psychopathology in healthy individuals, or demographic differences between the groups may explain absence of COMT X stress interaction in the healthy control group. Another possibility, however, would be that COMT Val158Met interactions with stress may be dependent on background genetic risk for psychotic disorder. Several studies have indeed reported epistatic interactions between COMT Val158Met and other candidate polymorphisms impacting on psychosis risk [33-35].

\section{Association emotional and psychotic reactivity}

Models of mediation showed that ESM psychosis was not a mediator of the ESM stress X COMT interaction in the model of NA, whereas NA did have a mediating effect in the model of ESM psychosis. This suggests that the effect on affective outcomes is primary, which may be consistent with the proposed role of stress-reactivity in what has been named the 'affective pathway to psychosis' [18]. In the model of momentary delusions, however, NA only partially mediated the interaction between COMT and ESM stress. Thus, while we found a major interactive effect of COMT and ESM stress on negative effect, there also was evidence for a specific role of the COMT $X$ stress interaction on delusional ideation independent from the association with negative affect. 


\section{Strengths and limitations}

Some methodological limitations are apparent. Measurements of momentary psychosis (delusions, hallucinations), NA, and event stress were based on subjective reports. Although it is sometimes assumed that subjective reports are less reliable than objective measures, they can be valid, whereas the validity of objective approaches should not be taken for granted [36]. Another issue in this regard is whether patients with a psychotic disorder were able to provide reliable and valid self-reports. Though, the prospective and real-time nature of ESM makes ESM assessments less prone to recall biases. This might be especially important in patients with psychosis as many of them display cognitive deficits [17]. Second, the current study used a daily life assessment technique in which participants had to comply with a paper-and-pencil diary protocol without the researcher being present. Recently, some authors have cast doubt on the reliability and subject compliance in paper-and-pencil ESM studies, favoring the use of electronic devices [37]. However, in a comparative study, Green et al. concluded that both methods yielded similar results [38]. Third, all analyses were cross-sectional, making it impossible to infer causality. Momentary psychosis and NA may be a reaction to daily stress or daily stress may result in an increase of momentary psychosis and NA. Nevertheless, the effect of COMT genotype on the association between stress and psychosis/NA holds in either case. The use of ESM is also a major strength of the current study, as it provides optimal measures of environmental factors for gene-environment interaction studies. ESM provides a prospective collection of cumulative, repeated measures of proximal environmental risk factors [10,11]. Another strength of the current study is that we included a general population as well as a patient sample and in this way were able to disentangle differential genetic effects between groups. Furthermore, cannabis users as well as no cannabis users were included in the study, thereby providing the possibility to test for the effect of cannabis use on the COMT-stress interaction. The relatively small sample size could have lead to undetected stratification biases. Amongst others, the uneven distribution of males and females between the patient and control sample may be an alternative explanation for the differences between patients and controls. In addition, undetected stratification for variables such as treatment duration, current treatment or symptom profile at intake could have influenced the results. However, since the present study is a replication of a previous study in an independent sample, it is unlikely that stratification for any one of these factors can account for the reported results.

\section{Conclusions}

Contingent on background liability for psychotic disorder, important differences may exist in the effect of COMT Val158Met on stress reactivity. Therefore, differential sensitivity to environmental stress occasioned by COMT Val158Met may be indicative of higher order interactions with genetic variation predisposing for psychotic disorder. 
Table 1. Demographic characteristics and descriptives

\begin{tabular}{|c|c|c|c|}
\hline & Patients ( $n=86)$ & Controls $(n=109)$ & \\
\hline Age, mean (SD) & $32.26(10.60)$ & $40.17(13.44)$ & Beta $=-3.96, P<0.001$ \\
\hline $\operatorname{Sex}(M / F)$ & $58 / 28(67 \% / 33 \%)$ & $35 / 74$ (32\%/68\%) & $\chi^{2}(1)=24.06, \mathrm{P}<0.001$ \\
\hline Education, n (\%) & & & $\chi^{2}(2)=23.08, \mathrm{P}<0.001$ \\
\hline Elementary school & $1(1.22 \%)$ & $0(0 \%)$ & \\
\hline Secondary school & $63(76.83 \%)$ & $48(44.04 \%)$ & \\
\hline Higher education & $18(21.95 \%)$ & $61(55.96 \%)$ & \\
\hline Living situation, n (\%) & & & $\chi^{2}(4)=58.37, P<0.001$ \\
\hline Alone & $19(22.62 \%)$ & 12 (11.01\%) & \\
\hline With partner & $19(22.62 \%)$ & $80(73.39 \%)$ & \\
\hline With parents or family & $18(21.43 \%)$ & $14(12.84 \%)$ & \\
\hline Protected housing & $21(25.00 \%)$ & $1(0.92 \%)$ & \\
\hline Others & $7(8.33 \%)$ & $2(1.83 \%)$ & \\
\hline ESM psychosis, mean (SD) & $1.59(0.79)$ & $1.18(0.29)$ & Beta $=0.21, P<0.001$ \\
\hline ESM delusions, mean (SD) & $1.73(0.92)$ & $1.27(0.44)$ & Beta $=0.23, P<0.001$ \\
\hline ESM hallucinations, mean (SD) & $1.32(0.84)$ & $1.0(0.02)$ & Beta $=0.16, P<0.001$ \\
\hline ESM stress, mean (SD) & $-1.34(0.87)$ & $-1.52(0.62)$ & Beta $=0.092, P=0.085$ \\
\hline ESM negative affect, mean (SD) & $1.92(0.95)$ & $1.34(0.36)$ & Beta $=0.29, P<0.001$ \\
\hline Cannabis use, current (y/n) & $37 / 35$ (51.4\%/48.6\%) & $7 / 65$ (90.3\%/9.7\%) & $\chi^{2}(1)=29.5, P<0.001$ \\
\hline Cannabis use, last 12 months (y/n) & $31 / 26(54.4 \% / 45.6 \%)$ & $7 / 13$ (35\%/65\%) & $\chi^{2}(1)=2.23, \mathrm{P}=0.14$ \\
\hline \multicolumn{4}{|l|}{ DSM IV diagnosis, No. (\%) } \\
\hline Schizophrenia/psychotic Disorder & $41(47.8 \%)$ & - & \\
\hline Schizoaffective disorder & $10(11.6 \%)$ & - & \\
\hline Other psychotic disorders & $35(40.7 \%)$ & - & \\
\hline No diagnosis & - & $109(100 \%)$ & \\
\hline
\end{tabular}

Note. Cannabis use, measured by the CIDI was available for 144 participants. 
Table 2. Sociodemographic characteristics and Experience Sampling Method (ESM) measures by genotype

\begin{tabular}{|c|c|c|c|c|c|c|c|c|}
\hline & \multicolumn{3}{|c|}{ Patients } & \multirow[b]{2}{*}{$P$} & \multicolumn{4}{|c|}{ Controls } \\
\hline & $\mathrm{Val} / \mathrm{Val}$ & Val/Met & Met/Met & & Val/Val & Val/Met & Met/Met & $P$ \\
\hline $\mathrm{n}$ & 20 & 46 & 20 & 0.25 & 28 & 46 & 35 & 0.25 \\
\hline Age (SD) & $31.1(8.2)$ & $33.4(11.5)$ & $30.9(10.7)$ & 0.97 & $39.8(14.3)$ & $41.7(13.6)$ & $38.4(12.7)$ & 0.64 \\
\hline Sex (M/F) & $13 / 7$ & $30 / 16$ & $15 / 5$ & 0.71 & $8 / 20$ & $15 / 31$ & $12 / 23$ & 0.89 \\
\hline $\begin{array}{l}\text { Number of ESM reports } \\
\text { (SD) }\end{array}$ & $42.1(10.1)$ & $39.4(8.6)$ & $39.3(9.2)$ & 0.33 & $47.2(8.3)$ & $47.8(7.5)$ & $45.9(8.7)$ & 0.48 \\
\hline ESM psychosis (SD) & $7.8(3.5)$ & $10.3(5.5)$ & $9.5(3.7)$ & 0.26 & $7.2(2.1)$ & $6.9(1.7)$ & $7.1(1.5)$ & 0.79 \\
\hline ESM delusions (SD) & $1.4(0.7)$ & $1.9(1.0)$ & $1.8(0.8)$ & 0.22 & $1.3(0.5)$ & $1.2(0.4)$ & $1.3(0.4)$ & 0.80 \\
\hline ESM hallucinations (SD) & $1.1(0.3)$ & $1.4(1.0)$ & $1.3(0.7)$ & 0.64 & $1.0(0.01)$ & $1.0(0.02)$ & $1.0(0.01)$ & 0.85 \\
\hline ESM stress (SD) & $-1.7(0.8)$ & $-1.3(0.8)$ & $-1.0(1.0)$ & 0.02 & $-1.4(0.7)$ & $-1.5(0.6)$ & $-1.6(0.6)$ & 0.24 \\
\hline ESM negative affect (SD) & $1.6(0.8)$ & $2.1(1.0)$ & $1.9(0.7)$ & 0.39 & $1.4(0.4)$ & $1.3(0.4)$ & $1.3(0.4)$ & 0.37 \\
\hline $\begin{array}{l}\text { Cannabis use, current } \\
\text { (yes/no) }\end{array}$ & $7 / 7$ & $18 / 14$ & $9 / 10$ & 0.81 & $0 / 17$ & $1 / 28$ & $5 / 17$ & 0.02 \\
\hline $\begin{array}{l}\text { Cannabis use, last } 12 \\
\text { months (yes/no) }\end{array}$ & $6 / 6$ & $14 / 11$ & $8 / 8$ & 0.91 & $0 / 4$ & $1 / 5$ & $5 / 4$ & 0.09 \\
\hline
\end{tabular}

Note. Cannabis use, measured by the CIDI was available for 144 participants. 
Table 3. Stratified effects of the interaction between COMT genotype and stress on affective and psychotic reactivity in psychosis patients and healthy controls

\begin{tabular}{|c|c|c|c|c|c|c|c|}
\hline & & \multicolumn{6}{|c|}{ COMT Val158Met genotype } \\
\hline & & \multicolumn{3}{|c|}{ Patients } & \multicolumn{3}{|c|}{ Controls } \\
\hline & & Val/Val & Val/Met & Met/Met & Val/Val & Val/Met & Met/Met \\
\hline NA & $\begin{array}{c}\beta^{a} \\
(95 \% \mathrm{Cl} ; \mathrm{p})\end{array}$ & $\begin{array}{c}0.09 \\
(0.05,0.13 ; p \\
<0.001)\end{array}$ & $\begin{array}{c}0.08 \\
(0.06,0.10 \\
p<0.001)\end{array}$ & $\begin{array}{c}0.15 \\
(0.11,0.18 \\
p<0.001)\end{array}$ & $\begin{array}{c}0.09 \\
(0.06,0.12 ; p< \\
0.001)\end{array}$ & $\begin{array}{c}0.08 \\
(0.06,0.10 \\
p<0.001)\end{array}$ & $\begin{array}{c}0.06 \\
(0.04,0.08 ; \\
p<0.001)\end{array}$ \\
\hline Psychosis & $\begin{array}{c}\beta^{\mathrm{b}} \\
(95 \% \mathrm{Cl} ; \mathrm{p})\end{array}$ & $\begin{array}{c}0.04 \\
(0.01,0.07 \\
p<0.05)\end{array}$ & $\begin{array}{c}0.04 \\
(0.02,0.05 \\
p<0.001)\end{array}$ & $\begin{array}{c}0.09 \\
(0.07,0.12 \\
p<0.001)\end{array}$ & $\begin{array}{c}0.01 \\
(-0.01,0.03 \\
p=0.3)\end{array}$ & $\begin{array}{c}0.02 \\
(0.01,0.04 \\
p<0.05)\end{array}$ & $\begin{array}{c}0.03 \\
(0.01,0.04 ; \\
p<0.05)\end{array}$ \\
\hline
\end{tabular}

${ }^{a}$ Regression coefficient indicates change in negative affect associated with changes in subjectively appraised stress.

${ }^{b}$ Regression coefficient indicates change in ESM psychosis associated with changes in subjectively appraised stress.

Note: The 2-way interactions in the control group were not significant. 
Figure 1: Effect sizes of event stress on NA: effect sizes of the NA response to event stress levels, stratified by COMTVal158Met genotype. ${ }^{*} P<0.001$ indicates that the difference between COMT Met/Met and COMT Val/Val \& Val/Met is significant.

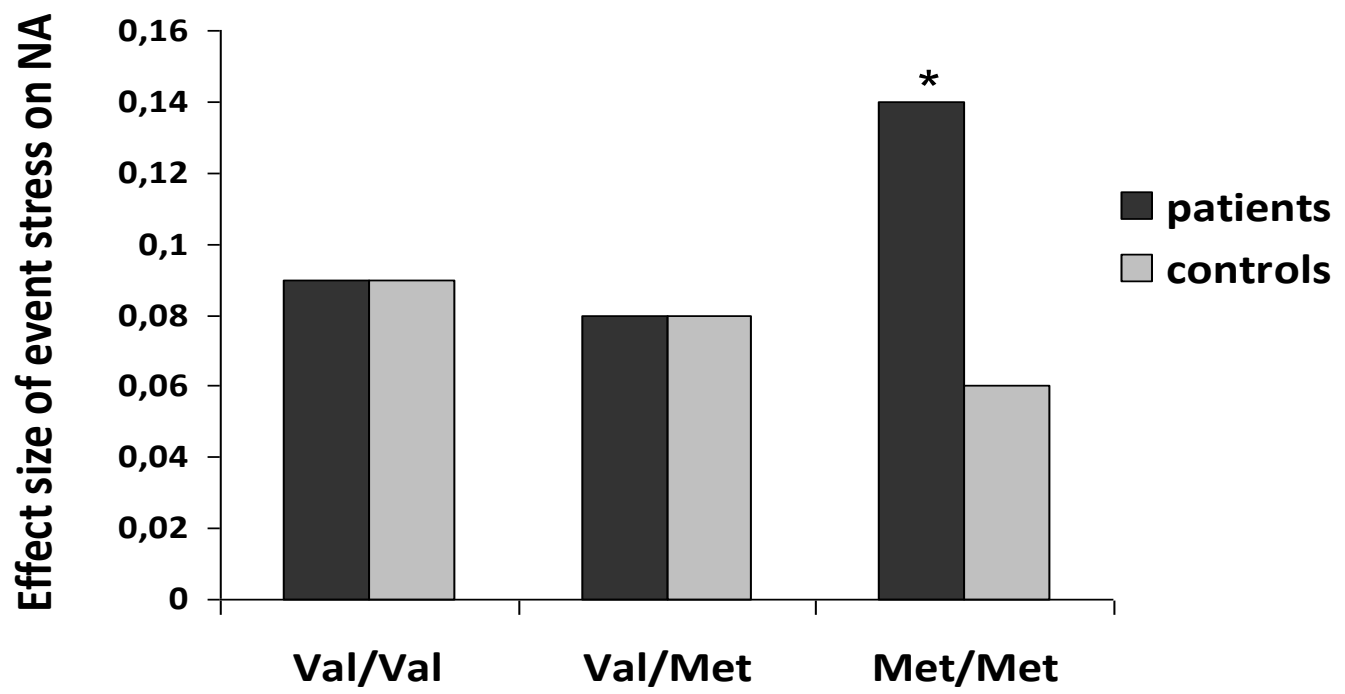

Figure 2: Effect sizes of event stress on momentary delusions in patients: effect sizes of the delusional responses of event stress levels 'pleasant' to 'very pleasant', stratified by COMT Val158Met genotype. There was a significant two-way interaction in the patient group $(2(4)=23.07, p<0.001)$.

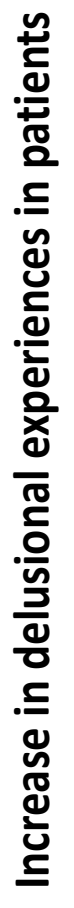

1,2

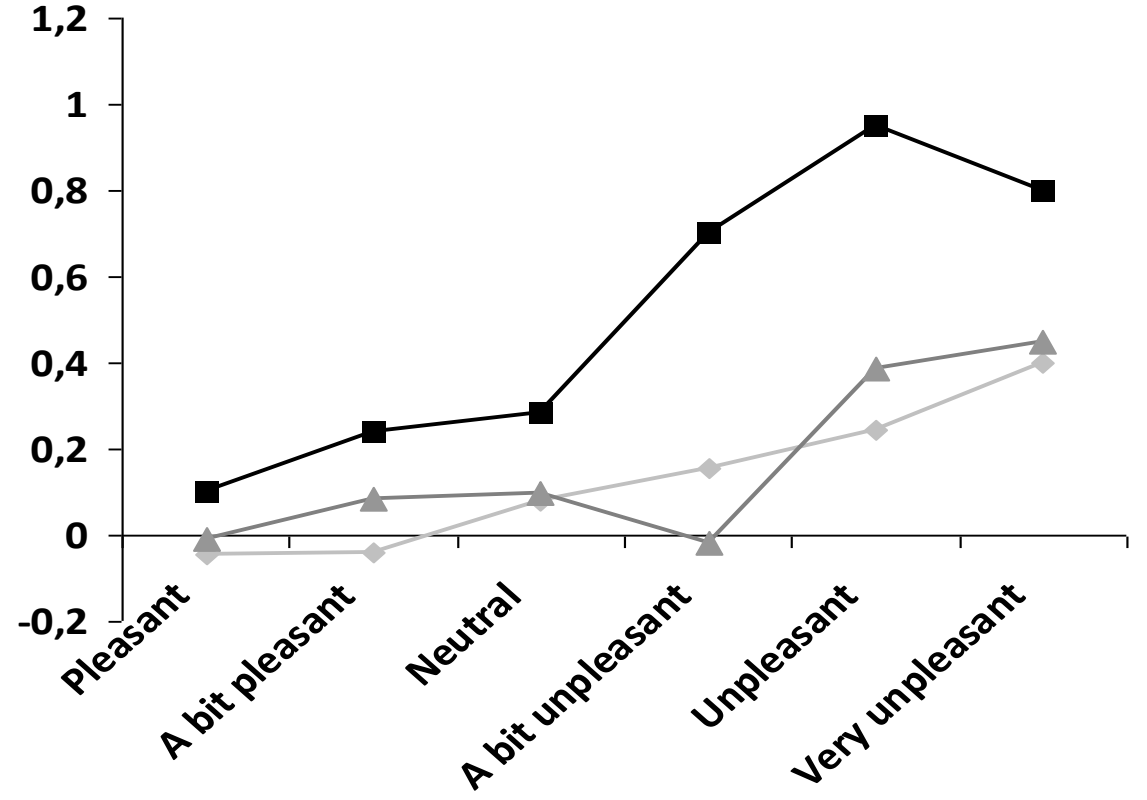

Met/Met

Val/Met

- Val/Val

Event stress 


\section{References}

1. Geuze E, Vermetten E, and Bremner JD (2005): MR-based in vivo hippocampal volumetrics: 2 . Findings in neuropsychiatric disorders. Mol Psychiatry 10:160-84.

2. Steen RG, Mull C, McClure R, Hamer RM, and Lieberman JA (2006): Brain volume in first-episode schizophrenia: systematic review and meta-analysis of magnetic resonance imaging studies. Br J Psychiatry 188:510-8.

3. Wright IC, Rabe-Hesketh S, Woodruff PW, David AS, Murray RM, and Bullmore ET (2000): Meta-analysis of regional brain volumes in schizophrenia. Am J Psychiatry 157:16-25.

4. Vita A, De Peri L, Silenzi C, and Dieci M (2006): Brain morphology in first-episode schizophrenia: a meta-analysis of quantitative magnetic resonance imaging studies. Schizophr Res 82:75-88.

5. Lawrie SM, Mclntosh AM, Hall J, Owens DG, and Johnstone EC (2008): Brain structure and function changes during the development of schizophrenia: the evidence from studies of subjects at increased genetic risk. Schizophr Bull 34:330-40.

6. Boos H, Aleman A, Cahn W, Pol HH, and Kahn RS, (2007): Brain volumes in relatives of patients with schizophrenia: a meta-analysis. Am Med Assoc. p. 297.

7. Corcoran C, Walker E, Huot R, Mittal V, Tessner K, Kestler L, et al. (2003): The stress cascade and schizophrenia: etiology and onset. Schizophr Bull 29:671-92.

8. O'Brien JT, (1997): The'glucocorticoid cascade'hypothesis in man: prolonged stress may cause permanent brain damage. Maney. p. 199-201.

9. Sapolsky RM, (2000): Glucocorticoids and hippocampal atrophy in neuropsychiatric disorders. Am Med Assoc. p. 925.

10. Buchanan TW, Tranel D, and Kirschbaum C (2009): Hippocampal damage abolishes the cortisol response to psychosocial stress in humans. Horm Behav 56:44-50.

11. Jacobson L and Sapolsky R (1991): The role of the hippocampus in feedback regulation of the hypothalamicpituitary-adrenocortical axis. Endocr Rev 12:118-34.

12. Lupien SJ, de Leon M, De Santi S, Convit A, Tarshish C, Nair NPV, et al., (1998): Cortisol levels during human aging predict hippocampal atrophy and memory deficits. Nature Publishing Group. p. 69-73.

13. Knoops AJG, Gerritsen L, van der Graaf Y, Mali W, and Geerlings MI, (2011): Basal Hypothalamic Pituitary Adrenal Axis Activity and Hippocampal Volumes: The SMART-Medea Study. Elsevier.

14. Mondelli V, Dazzan P, Hepgul N, Di Forti M, Aas M, D’Albenzio A, et al. (2010): Abnormal cortisol levels during the day and cortisol awakening response in first-episode psychosis: the role of stress and of antipsychotic treatment. Schizophr Res 116:234-42.

15. Pruessner JC, Dedovic K, Pruessner M, Lord C, Buss C, Collins L, et al. (2008): Stress regulation in the central nervous system: evidence from structural and functional neuroimaging studies in human populations - 2008 Curt Richter Award Winner. Psychoneuroendocrinology 35:179-91.

16. Tessner KD, Walker EF, Dhruv SH, Hochman K, and Hamann S, (2007): The relation of cortisol levels with hippocampus volumes under baseline and challenge conditions. Elsevier. p. 70-78.

17. Myin-Germeys I, Delespaul P, and van Os J (2005): Behavioural sensitization to daily life stress in psychosis. Psychol Med 35:733-41.

18. Myin-Germeys I, van Os J, Schwartz JE, Stone AA, and Delespaul PA (2001): Emotional reactivity to daily life stress in psychosis. Arch Gen Psychiatry 58:1137-44.

19. Pruessner M, Pruessner JC, Hellhammer DH, Bruce Pike G, and Lupien SJ (2007): The associations among hippocampal volume, cortisol reactivity, and memory performance in healthy young men. Psychiatry Res 155:1-10.

20. G.R.O.U.P. (2011): Evidence that Familial Liability for Psychosis is Expressed as Differential Sensitivity to Cannabis: an Analysis of Patient-Sibling and Sibling-Control Pairs. Arch Gen Psychiatry in press. 
21. Maxwell ME (1992): Family Interview for Genetic Studies (FIGS): Manual For FIGS. 1992, Clinical Neurogenetics Branch, Intramural Research Program, National Institute of Mental Health, Bethesda, MD.

22. APA (2000): Diagnostic and statistical manual of mental disorders. 4th ed. 2000, Washington, DC: American Psychiatric Association.

23. Andreasen NC, Flaum M, and Arndt S (1992): The Comprehensive Assessment of Symptoms and History (CASH). An instrument for assessing diagnosis and psychopathology. Arch Gen Psychiatry 49:615-23.

24. Kay SR, Fiszbein A, and Opler LA (1987): The positive and negative syndrome scale (PANSS) for schizophrenia. Schizophr Bull 13:261-76.

25. Cahn W, Pol HE, Lems EB, van Haren NE, Schnack HG, van der Linden JA, et al. (2002): Brain volume changes in firstepisode schizophrenia: a 1-year follow-up study. Arch Gen Psychiatry 59:1002-10.

26. WHO, (1990): Composite International Diagnostic Interview (CIDI). World Health Organization: Geneva.

27. Csikszentmihalyi M and Larson R (1987): Validity and reliability of the Experience-Sampling Method. J Nerv Ment Dis 175:526-36.

28. Myin-Germeys I, Oorschot M, Collip D, Lataster J, Delespaul P, and van Os J (2009): Experience sampling research in psychopathology: opening the black box of daily life. Psychol Med 39:1533-47.

29. Delespaul P, deVries M, and van Os J (2002): Determinants of occurrence and recovery from hallucinations in daily life. Soc Psychiatry Psychiatr Epidemiol 37:97-104.

30. Jacobs N, Myin-Germeys I, Derom C, Delespaul P, van Os J, and Nicolson NA (2007): A momentary assessment study of the relationship between affective and adrenocortical stress responses in daily life. Biol Psychol 74:60-6.

31. Sulon J, Demey-Ponsart L, Beauduin P, and Sodoyez JC (1978): Radioimmunoassay of corticosterone, cortisol and cortisone: their application to human cord and maternal plasma. J Steroid Biochem 9:671-6.

32. Dale AM, Fischl B, and Sereno MI (1999): Cortical surface-based analysis. I. Segmentation and surface reconstruction. Neuroimage 9:179-94.

33. Fischl B and Dale AM (2000): Measuring the thickness of the human cerebral cortex from magnetic resonance images. Proc Natl Acad Sci U S A 97:11050-5.

34. Fischl B, Salat DH, Busa E, Albert M, Dieterich M, Haselgrove C, et al. (2002): Whole brain segmentation: automated labeling of neuroanatomical structures in the human brain. Neuron 33:341-55.

35. Fischl B, Sereno MI, and Dale AM (1999): Cortical surface-based analysis. II: Inflation, flattening, and a surfacebased coordinate system. Neuroimage 9:195-207.

36. Han X, Jovicich J, Salat D, van der Kouwe A, Quinn B, Czanner S, et al. (2006): Reliability of MRI-derived measurements of human cerebral cortical thickness: the effects of field strength, scanner upgrade and manufacturer. Neuroimage 32:180-94.

37. Jovicich J, Czanner S, Greve D, Haley E, van der Kouwe A, Gollub R, et al. (2006): Reliability in multi-site structural MRI studies: effects of gradient non-linearity correction on phantom and human data. Neuroimage 30:436-43.

38. Segonne F, Dale AM, Busa E, Glessner M, Salat D, Hahn HK, et al. (2004): A hybrid approach to the skull stripping problem in MRI. Neuroimage 22:1060-75.

39. StataCorp (2009): STATA/SE statistical software, release 11. 2009, Texas: College Station.

40. Meador-Woodruff JH and Greden JF, (1988): Effects of psychotropic medications on hypothalamic-pituitary-adrenal regulation. p. 225.

41. Seidman LJ, Faraone SV, Goldstein JM, Kremen WS, Horton NJ, Makris N, et al. (2002): Left hippocampal volume as a vulnerability indicator for schizophrenia: a magnetic resonance imaging morphometric study of nonpsychotic first-degree relatives. Vol. 59. 2002: Am Med Assoc. 839.

42. Gunduz-Bruce H, Szeszko PR, Gueorguieva R, Ashtari M, Robinson DG, Kane JM, et al., (2007): Cortisol levels in relation to hippocampal sub-regions in subjects with first episode schizophrenia. Elsevier. p. 281-287. 
43. Mondelli V, Pariante CM, Navari S, Aas M, D’Albenzio A, Di Forti M, et al. (2010): Higher cortisol levels are associated with smaller left hippocampal volume in first-episode psychosis. Schizophr Res 119:75-78.

44. Lataster T, Collip D, Lardinois M, van Os J, and Myin-Germeys I (2010): Evidence for a familial correlation between increased reactivity to stress and positive psychotic symptoms. Acta Psychiatr Scand 122:395-404.

45. Myin-Germeys I and van Os J (2007): Stress-reactivity in psychosis: evidence for an affective pathway to psychosis. Clin Psychol Rev 27:409-24.

46. Szeszko PR, Betensky JD, Mentschel C, Gunduz-Bruce H, Lencz T, Ashtari M, et al. (2006): Increased stress and smaller anterior hippocampal volume. Neuroreport 17:1825-8.

47. Stone AA, Shiffman S, Schwartz JE, Broderick JE, and Hufford MR (2002): Patient non-compliance with paper diaries. BMJ 324:1193-4.

48. Jacobs N, Nicolson NA, Derom C, Delespaul P, van Os J, and Myin-Germeys I (2005): Electronic monitoring of salivary cortisol sampling compliance in daily life. Life Sciences76:2431-43.

49. Green AS, Rafaeli E, Bolger N, Shrout PE, and Reis HT (2006): Paper or plastic? Data equivalence in paper and electronic diaries. Psychol Methods 11:87-105.

50. Hruschka DJ, Kohrt BA, and Worthman CM (2005): Estimating between- and within-individual variation in cortisol levels using multilevel models. Psychoneuroendocrinology 30:698-714. 
Chapter 7 



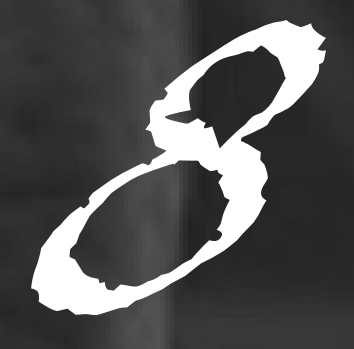

Epilogue 


\section{On the pathway from social factors to psychosis}

The first aim of the current thesis was to examine the role and mechanisms of social factors in the development of clinical and subclinical positive psychotic symptoms (chapter 2, 3 and 4). To elucidate some of the processes involved, we chose two approaches: an epidemiological approach, in which we studied the longitudinal association between interpersonal functioning and different subclinical positive psychotic symptom dimensions, and a momentary assessment approach, in which we zoomed in into the microenvironment to study the role of momentary social contexts on clinical and subclinical paranoia.

The significance of social and interpersonal functioning in the development of psychotic disorders is widely acknowledged. However, less is known about potential differences in the association of interpersonal functioning with different symptom dimensions of subclinical psychotic experiences. In chapter $\mathbf{3}$ we addressed the bidirectional, longitudinal association between dimensions of subclinical positive psychotic experiences and interpersonal functioning in an adolescent general population sample. Results show that a decline in interpersonal functioning may form the starting point of gradually more severe expression of psychotic experiences, in a context- and symptom-specific fashion. Only bizarre experiences and persecutory ideation displayed consistent longitudinal associations with interpersonal functioning. Moreover, differences in the association with subclinical symptoms may exist between different interpersonal contexts. Peer functioning primarily predicted bizarre experiences and family functioning was associated with persecutory ideation demonstrating a vicious cycle in which subclinical persecutory ideation and family functioning impact one another.

The link between interpersonal functioning and persecutory ideation is in line with evidence from the paranoia literature. Especially, experimental studies suggest an association between ambiguous social environments and paranoia [1]. Virtual reality paradigms that examined paranoia in different ambiguous social contexts found increasing levels of paranoia in individuals with paranoid thinking patterns [2]. To increase the understanding of the mechanisms between social context and paranoia at different levels of the extended paranoia phenotype, we investigated short-term fluctuations in paranoid ideations dependent on real-life social situations using a momentary assessment approach (chapter 4). Momentto-moment changes in paranoia were context dependent in individuals with low or at-risk levels of trait paranoia, but were independent of social company in individuals with high trait paranoia, suggesting qualitative differences between individuals with low, medium and high levels of trait paranoia. However, general levels of subjective distress were important predictors of paranoid thinking at all levels of trait paranoia, and indeed the most paranoid individuals showed the greatest reactivity to subjective stress, suggesting a dose-response effect on paranoid thinking. Thus, the qualitative differences we found between paranoia groups seem to be specific for the association between paranoia and social company, which is a relatively 'objective' contextual measure. These findings suggest that, for highly paranoid individuals, subjective experiences of stress have become decoupled from social context. 
What might be the mechanisms involved? Sensitivity to social context in the low and medium paranoia groups might reflect adaptive or even protective mechanisms: when exposed to unfamiliar people, an individual may be inclined to be wary and suspicious of their intentions, but when encountering someone with whom they have a long history of positive interactions, wariness may not be warranted. The failure to take into account this social contextual information may be a core feature of severe paranoid delusions. Additionally, the absence of these protective mechanisms in high trait paranoid individuals might put them at increased risk to actually encounter unpleasant social interactions, thereby resulting in even more wariness and suspicion. In fact, their interactions with familiar individuals might indeed be characterized by uncertainty or insecurity. This can also be interpreted in light of the findings presented in chapter 3. Poor interpersonal functioning predicted an increase in persecutory ideation later on. Maybe, high paranoid individuals have never experienced a safe family environment and therefore have never learned to "differentiate" the safety of being with familiar and non-familiar social company. Poor interpersonal functioning, in turn, might be the result of exposure to early adversity [3].

In future studies, it will be essential to also measure environmental exposures (e.g. migration, childhood trauma), as such environmental risk factors may interact with developmental processes potentially resulting in abnormal dopamine sensitization or cognitive biases and thereby creating enduring liabilities for psychotic disorder (chapter 2). These, in interaction with interpersonal problems during adolescence, might result in the persistence of subclinical normally transitory symptoms, and thereby increase the risk to overt psychotic disorder [4]. Furthermore, it should be examined whether the direction of the association for interpersonal functioning and psychotic experiences are specific for adolescent population samples or translate to other ages and stages of the psychosis continuum.

Many questions with respect to the link between environmental risk factors and development of psychosis remain. However, the current thesis suggests that exposure to stressful environments, especially those being characterized by social defeat processes, in interaction with (epi)genetic factors can alter psychological and physiological processes (chapter 2 ). These alterations can be traced to a final common pathway of cognitive biases and altered dopamine neurotransmission, which can be

referred to as "sensitization". Such sensitization processes can facilitate the onset and persistence of psychotic symptoms.

\section{Biological and genetic markers of stress reactivity in psychosis}

A second aim of the current thesis was to examine biological and genetic markers of stress reactivity in psychosis. Alterations in HPA axis activity, including changes in hippocampal volume size and cortisol secretory patterns, were investigated as underlying marker for psychosis risk and increased daily life stress reactivity (chapter 5 and 6). Moreover, we investigated the association between variations in COMT genotype and stress sensitivity as a function of genetic risk for psychotic disorder (chapter 7). 
HPA axis

Aberrations in brain volume, including hypothalamus, pituitary and hippocampus [5-7], and HPA axis functioning, comprising cortisol levels and reactivity to stress [8-10], are commonly reported in patients with psychotic disorders. Furthermore, studies have reported structural HPA axis alterations in relatives of patients with schizophrenia. Yet, cortisol secretory patterns or the possible association of cortisol with emotional and psychotic reactivity to stress in daily life have never been examined. In the study described in chapter 5, we report that siblings of individuals with psychotic disorder had higher mean cortisol levels throughout the day and greater cortisol reactivity to daily stressors than a comparison group with no family history of psychosis. In the sibling but not the control group, momentary psychotic experiences and negative emotions were associated with transient increases in cortisol secretion. These findings support the notion that altered activity of the HPA axis may be a marker of underlying vulnerability for psychotic disorder. Subsequently, we extended these findings by examining the role of hippocampal volume on HPA axis and emotional reactivity to stress in a sample with different levels of genetic risk for psychosis (chapter 6). Results show that the immediate effect of daily stress on negative affect and cortisol was conditional on hippocampal volume, but also on genetic risk for psychosis. Patients with a psychotic disorder were not only more likely to have a small hippocampus, but those with smaller left hippocampal volume were also more likely to experience augmented emotional stress reactivity compared to patients with large left hippocampal volume. In line with the results in patients, siblings with small hippocampal volume demonstrated increased emotional and cortisol reactivity to stress compared to those with large hippocampal volume. In contrast, controls with a large total hippocampal volume were more emotionally stress reactive than controls with small total hippocampal volume. The findings in individuals with a genetic risk for psychosis support the evidence that the hippocampus and in particular the left hippocampus [20] has an inhibitory role on the human stress response, extending previous findings to the realm of daily life. The discrepant association between size of the hippocampus and degree of stress reactivity in control and risk groups poses many new questions (see "Mechanisms along the psychosis continuum"). Nevertheless, they are in line with the results of chapter 7, in which we found that catechol-O-methyltransferase (COMT) Val158Met interactions with stress were contingent on background genetic risk for psychotic disorder.

\section{Gene-environment interactions}

An earlier ESM study has suggested that psychotic experiences in response to minor stressors may be functional states of an abnormal dopamine reactivity [11]. Genetic variation in the Val158Met functional polymorphism of the COMT gene was therefore an obvious candidate to study genetic moderation of environmental stress in psychosis. A major aim of this study was to elucidate inconclusive findings regarding the COMT-stress interactions in psychosis [12-14]. Our results demonstrated differences between patients and healthy controls in the effect of COMT genotype on stress reactivity. Patients with the Met/Met genotype showed significantly increased psychotic and emotional reactivity to stress 
in comparison to patients with the Val/Met and Val/Val genotypes. In contrast, healthy controls did not display large or significant COMT Val158Met-stress interactions. Thus, differences might exist in the effect of COMT Val158Met on stress reactivity, which may depend on background risk for psychotic disorder.

Differential effects of COMT activity have been associated with the Val and Met allele. Animal research suggests that affective functioning is deteriorated in mice with a COMT disruption. On the other hand, these mice seem to have advantages regarding working memory performance [15]. This trade-off between executive cognition and emotion regulation between different COMTVal158Met genotypes has also been replicated in human studies. A meta-analysis showed that Met/Met carriers performed better on executive functioning tasks, but worse on emotion regulation than Val/Val carriers [16-18]. Moreover, Met-allele carriers showed an increased prefrontal activation after stress exposure [16]. However, also the sensitivity to positive aspects in the environment, like the ability to experience reward in daily life, increases with the number of 'Met' alleles of an individual [19]. As suggested by Belsky and colleagues, it thus seems that we should not think of COMT in terms of a 'vulnerability gene', but more in terms of a 'plasticity gene' [20]. Under conditions of adverse environment, like trauma or life events, repeated exposure of stress may result in sensitization of prefrontal activation in Met allele carriers, while when not exposed to adversity, Met allele carriers might even be better off than Val allele carriers with regard to stress reactivity. This pattern is reflected in the results of chapter 7. Met/Met patients, compared to Met/Met controls, have probably experienced relatively more trauma and stressful life events [21] and were more stress-reactive than Val/Val patients. On the other hand, in the control group, which as a whole was probably less exposed to traumatic events, the opposite pattern emerged. Val/Val control participants were relatively more stress reactive than Met/Met control participants, supporting the notion of COMT as a 'plasticity gene'.

Interestingly, effect sizes for the Val/Val and Val/Met carriers were about the same in patients and controls and most prominent were the larger effect sizes in Met/Met patients. Since some genotypes are more sensitive to epigenetic (environmental impact on gene expression) changes [22], it is attractive to speculate that Met/Met carriers of COMT might be more sensitive to epigenetic mechanisms. While we found Met/Met carriers to be more psychotically reactive to stress, other studies reported the opposite with amplified psychotic stress-reaction in Val/Val relative to Met carriers [13, 14]. Interestingly, these other studies were conducted in general population samples assessing subclinical psychotic experiences. The direction of the effect in our control group, although not statistically significant, was comparable to these results with numerically larger increases in symptoms after stress exposure in the Val/Val carriers compared to the Met/Met carriers. A further observation is that the reported effect sizes for the Val/Val and $\mathrm{Val} /$ Met carriers were similar in patients and controls whereas a large difference in effect size was found in Met/Met patients compared to Met/Met controls.

COMT expression is highly dependent on its methylation status [23] and interactions with MTHFR C677T, which is known to affect availability of methyl groups for DNA methylation, have been reported [24]. 
Therefore, it could be hypothesized that the large difference in effect size between Met/Met patients and Met/Met controls is underpinned by differences in COMT methylation. Previous research indeed suggested that COMT hypomethylation may be a risk factor for psychosis [23]. Furthermore, differential DNA methylation based on genotype has been previously reported. For example, individuals with the Val/Val of the Brain Derived Neurotrophic Factor (BDNF) Val66Met polymorphism displayed more exonic DNA methylation than did BDNF Met-carriers [25]. Thus, genotype-specific environmentally-induced differences in DNA methylation may potentially blur the distinction between 'gene-environment interaction' and 'epigenetics' and complicate statistical models of GxE that assume a non-changing, predictable effect of alleles on protein expression and function [22].

Thus background genetic factors that interact with COMT Val158Met may shape the interaction between COMT Val158Met and environmental stress and may explain differences between patient groups and healthy controls. Thus, when testing gene-environment interactions with one single measure of molecular genetic variation, the net background genetic risk for psychosis should be considered as well [26].

To conclude, patients with a Met/Met genotype of COMTVal158Met seem to be more prone to stress, probably paralleled by increased dopamine release in response to stress, which in turn result in increased cortisol secretion. Chronic cortisol secretion can damage the hippocampus, which may result in a sensitized HPA axis activity (i.e. more cortisol secretion), as a result of a deterioration of the inhibitory feedback mechanism of the hippocampus. Increased cortisol secretion in turn may facilitate striatal dopamine release thereby increasing the risk for the development of psychotic experiences.

\section{Mechanisms along the psychosis continuum}

Some of the findings of the current thesis suggest that, superimposed on the continuity of psychosis [27], there may be qualitative differences between those with severe psychotic experiences and those with less severe psychotic experiences. Results of chapter 4 (differential effects of social company on low and medium versus high levels of paranoia), chapter 6 (differential effect of hippocampal volume in patients and siblings versus controls), and chapter 7 (differential effect of COMT Met/Met genotype in patients versus controls) challenge earlier studies that suggest an etiological continuity of psychotic symptoms between subclinical and more advanced levels of symptom expression [28]. According to the latter, the differences in the reaction to the environment between groups would mainly be of quantitative character. However, considering the results of the present thesis, it seems that there are some qualitative differences in the mechanisms involved in patients with a psychotic disorder compared to those without genetic risk for psychotic disorder.

Some of the discrepancies in the association between stress reactivity and biological markers between 
patients with a psychotic disorder and control groups might be explained by sensitization processes (chapter 2). As discussed earlier (see "Gene-environment interactions") environmental factors that may be more prevalent in individuals with psychotic disorder, for instance childhood trauma [21], may change the sensitivity of the (stress) system, possibly through epigenetic changes. A good example comes from the PTSD literature. A polymorphism in FKBP5, which is a modulator of glucocorticoid sensitivity, influences the association between childhood trauma and later development of posttraumatic stress. The effects of FKBP5 are reversed in those who have experienced trauma [29]. Similarly, differences in FKBP5 may influence the effect of childhood trauma on stress/HPA axis sensitivity. One might speculate, that patients with smaller hippocampal volume may have experienced childhood trauma, impacting on cumulative glucocorticoid exposure and hippocampal volume. Hippocampal volume in turn, as shown in the current study, influences stress-reactivity.

\section{Biological alterations in those at increased genetic risk - sign of shared environment, psychosis liability or resilience?}

Generalization of the results of chapter 5 and 6, which included an adult sibling sample, to other groups at increased (genetic) risk for psychosis should be done cautiously. Compared to children of parents with a psychotic disorder and others identified as being at ultra high risk for developing a disorder, the siblings included in our studies were older, in most cases past the age when onset of psychosis is most likely to occur. The nature and extent of HPA axis abnormalities in healthy siblings may differ from those described in at-risk and ultra high risk adolescent samples. Therefore, it cannot be eliminated that the increased cortisol reactivity in our sibling group reflects a protective rather than a risk factor. On the other hand, even with a suboptimal biological reactivity, siblings may manage to cope better with stress and/or have other resilience factors protecting them from psychotic disorder.

Another issue that should be considered is that siblings share not only their genes with their ill brothers and sisters but also the early rearing environment. Thus, childhood adversity - which has been associated with psychotic disorder [30] and HPA axis abnormalities [31, 32]- could have influenced the results in chapter 5 and 6 . However, we found no differences in traumatic experiences between siblings and control participants, suggesting that other factors may explain differences in cortisol secretion between groups.

Although we found evidence for an association between the amount of cortisol secretion and subclinical psychotic experiences in unaffected first-degree relatives, we could not establish whether this association is causal. Thus, the observed interrelationships among cortisol, psychotic experiences and negative emotions need to be further explored in longitudinal or experimental designs. Longitudinal designs can answer the question whether cortisol alterations in first degree relatives is rather a reflection of resilience or rather of their genetic vulnerability to psychotic disorder. Most importantly, longitudinal 
studies should examine in how far cortisol reactivity to stress relates to the development of psychotic symptoms in individuals at risk; thus whether increased cortisol reactivity predicts symptom formation rather than symptoms resulting in stress-related increases of cortisol. Moreover, studies that either induce stress and measure subsequent changes in psychotic symptoms and emotions or that induce psychotic experiences and subsequent changes in cortisol are needed to answer the question of causality more conclusively.

Furthermore, we found a decreased emotional response to stress in both siblings and patients with larger hippocampal volume. Additionally, in siblings, larger hippocampal volume was associated with a reduced cortisol response to stress. These may represent markers for reduced integration of the stress response in individuals at increased risk for psychosis. In other words, a decreased emotional response and decreased cortisol concentrations in the siblings with larger hippocampal volume, possibly reflecting suboptimal HPA axis functioning, may be a sign of a suboptimal response to psychosocial stress, resulting in increased liability for psychosis. Another possibility is that the blunted cortisol stress response in siblings with larger hippocampal volume represents a protective factor against illness expression, given increased background vulnerability. However, the fact that we found similar patterns between stress reactivity and hippocampal volume in the patients and the siblings argues for an interpretation as a sign of psychosis vulnerability.

Nevertheless, the direction of the association between hippocampal volume and stress reactivity remains unresolved. The question of whether heightened cortisol reactivity may ultimately cause hippocampal volume changes or whether alterations in hippocampal volume, possibly through decreased inhibitory functioning of the hippocampus, are responsible for increased stress reactivity, should be answered in longitudinal studies, preferably starting at an age before adolescence. Moreover, longitudinal studies should disentangle factors of shared environment, psychosis liability and resilience in more detail.

\section{Clinical Implications}

More insight in the mechanisms that determine increasing levels of subclinical psychotic experiences or their persistence may offer valuable insights regarding early intervention. Several studies, including the one presented in chapter 3 , suggest that deteriorations in social functioning increases the likelihood of future subclinical symptoms, that again increase the risk for psychotic disorder. As suggested in this thesis, certain types of subclinical psychotic symptoms are more relevant than others in the early association with interpersonal functioning. Therefore, preventive strategies that predominantly target the experience of subclinical bizarre experiences and persecutory ideations and evaluate them in association with peer and family functioning may be useful.

Moreover, the autonomy of paranoid ideas with respect to different social contexts, which we found 
in high-paranoid individuals, may have implications for the clinical management of paranoid patients. Cognitive behavioral therapy for paranoid symptoms usually involves encouraging patients to reflect on the social context of their symptoms and question their interpretations of events [33]. If paranoid thoughts are autonomous of social context, patients may have difficulty doing this. It seems possible that approaches that encourage detached acceptance of unpleasant thoughts, for example acceptance and commitment therapy, will be more effective. A small randomized controlled trail has reported positive effects of acceptance commitment therapy for patients recovering from acute psychosis [34].

Earlier studies have suggested that impairments in cognition are independent or even mutually exclusive from increased stress reactivity in patients with a psychotic disorder, suggesting that increased stress sensitivity might be expressed by an independent subgroup of patients with psychotic disorder [35, 36]. However, so far no diagnostic tools exist to determine which patients "went the affective pathway to psychosis", i.e. for which patients the psychotic experiences can probably be traced back to detrimental stress reactions. Certainly, clinical interviews that map the lives and experiences of patients are indispensable, but additional markers of the stress sensitive subtype of psychotic disorder might help to improve identification of appropriate clinical management, tailor treatment to the underlying etiology and advance the understanding of the underlying mechanisms involved. We identified "biomarkers" for differences in stress reactivity within the group of patients with psychotic disorders, i.e. patients with smaller left hippocampal volume and the Met/Met genotype of the COMT gene were most responsive to stressful environments. Interestingly, however, in patients with schizophrenia, the Val allele in contrast to the Met allele has been associated with decreased brain volume and worse cognition [37, 38], both characteristics of a more chronic course, worse outcome and neurodevelopmental pathway to psychosis [35]. Thus, this finding underscores the notion that there might be different pathways to psychotic disorder that are associated with different biological and genetic markers, the stress related pathway being one of them $[39,40]$.

In the cancer literature a paradigm shift is happening, from treating all patients according to standard guidelines to individualized molecular targeted treatments. Information flow through the intra- and extracellular molecular protein networks that interconnect organ and circulatory systems together is characterized and can be both new targets for therapeutics themselves as well as represent diagnostic biomarkers [41, 42]. Similarly, advancing our understanding of the different subtypes of psychotic disorders might help to tailor treatments. This is still far from offering direct therapeutic benefit and additional "biomarkers" or "behavioral markers" of the affective pathway to psychosis/different etiological subgroups of psychotic patients need to be identified. However, in the future, tailored and individualized treatments according to underlying mechanism of vulnerability, for instance teaching those with increased stress reactivity to better cope with stress, might be useful.

In this regard, another approach that promises possibilities for even more individualized treatments is the use of real-world momentary assessment electronic devices (e.g. PsyMate; computerized ESM) [43]. Summary information on the association of internal and external momentary cues on symptom 
expression (e.g. effect of social company on paranoia as discussed in chapter 4, but also fluctuations in anxiety and self-esteem [44, 45]), can be given as feedback to patients. This might increase their awareness of the processes that are involved in the formation of their symptoms [43]. This feedback could be also given in real-time and may introduce behavioral suggestions to patients, when they are dealing with a potentially "symptom evoking" external (e.g. certain environment) or internal situation (e.g. increase in anxiety). These kind of approaches will increase the involvement of patients in their own therapeutic process [43]. 


\section{References}

1. Ellett L, Freeman D, and Garety PA (2008): The psychological effect of an urban environment on individuals with persecutory delusions: The Camberwell walk study. Schizophr Res.

2. Freeman D, Slater M, Bebbington PE, Garety PA, Kuipers E, Fowler D, et al. (2003): Can virtual reality be used to investigate persecutory ideation? J Nerv Ment Dis 191:509-14.

3. Mueser KT, Rosenberg SD, Goodman LA, and Trumbetta SL (2002): Trauma, PTSD, and the course of severe mental illness: an interactive model. Schizophrenia Research 53:123-143.

4. Dominguez MD, Wichers M, Lieb R, Wittchen HU, and van Os J Evidence that onset of clinical psychosis is an outcome of progressively more persistent subclinical psychotic experiences: an 8-year cohort study. Schizophr Bull 37:84-93.

5. Pariante CM (2008): Pituitary volume in psychosis: the first review of the evidence. Journal of Psychopharmacology 22:76-81.

6. Wright IC, Rabe-Hesketh S, Woodruff PW, David AS, Murray RM, and Bullmore ET (2000): Meta-analysis of regional brain volumes in schizophrenia. American Journal of Psychiatry 157:16-25.

7. Koolschijn PC, van Haren NE, Hulshoff Pol HE, and Kahn RS (2008): Hypothalamus volume in twin pairs discordant for schizophrenia. European Neuropsychopharmacology 18:312-5.

8. Jansen LM, Gispen de Wied CC, Gademan PJ, De Jonge RC, van der Linden JA, and Kahn RS (1998): Blunted cortisol response to a psychosocial stressor in schizophrenia. Schizophr Res 33:87-94.

9. Marcelis M, Cavalier E, Gielen J, Delespaul P, and Van Os J (2004): Abnormal response to metabolic stress in schizophrenia: marker of vulnerability or acquired sensitization? Psychological Medicine 34:1103-11.

10. Ryan MC, Sharifi N, Condren R, and Thakore JH (2004): Evidence of basal pituitary-adrenal overactivity in first episode, drug naive patients with schizophrenia. Psychoneuroendocrinology 29:1065-70.

11. Myin-Germeys I, Marcelis M, Krabbendam L, Delespaul P, and van Os J (2005): Subtle fluctuations in psychotic phenomena as functional states of abnormal dopamine reactivity in individuals at risk. Biol Psychiatry 58:105-10.

van Winkel R, Henquet C, Rosa A, Papiol S, Fananas L, De Hert M, et al. (2008): Evidence that the COMT(Val158Met) polymorphism moderates sensitivity to stress in psychosis: an experience-sampling study. Am J Med Genet B Neuropsychiatr Genet 147B:107.

13. Simons CJ, Wichers M, Derom C, Thiery E, Myin-Germeys I, Krabbendam L, et al. (2009): Subtle gene-environment interactions driving paranoia in daily life. Genes Brain Behav 8:5-12.

14. Stefanis NC, Henquet C, Avramopoulos D, Smyrnis N, Evdokimidis I, Myin-Germeys I, et al. (2007): COMT Val158Met moderation of stress-induced psychosis. Psychol Med 37:1651-6.

15. Papaleo F, Crawley JN, Song J, Lipska BK, Pickel J, Weinberger DR, et al. (2008): Genetic dissection of the role of catechol-Omethyltransferase in cognition and stress reactivity in mice. J Neurosci 28:8709-23.

16. Mier D, Kirsch P, and Meyer-Lindenberg A (2009): Neural substrates of pleiotropic action of genetic variation in COMT: a metaanalysis. Mol Psychiatry.

17. Smolka MN, Schumann G, Wrase J, Grusser SM, Flor H, Mann K, et al. (2005): Catechol-O-methyltransferase val158met genotype affects processing of emotional stimuli in the amygdala and prefrontal cortex. J Neurosci 25:836-42.

18. Drabant EM, Hariri AR, Meyer-Lindenberg A, Munoz KE, Mattay VS, Kolachana BS, et al. (2006): Catechol O-methyltransferase val158met genotype and neural mechanisms related to affective arousal and regulation. Arch Gen Psychiatry 63:1396-406.

19. Wichers M, Aguilera M, Kenis G, Krabbendam L, Myin-Germeys I, Jacobs N, et al. (2008): The catechol-O-methyl transferase Val158Met polymorphism and experience of reward in the flow of daily life. Neuropsychopharmacology 33:3030-6.

20. Belsky J, Jonassaint C, Pluess M, Stanton M, Brummett B, and Williams R (2009): Vulnerability genes or plasticity genes? Mol Psychiatry 14:746-54.

21. Read J, Perry BD, Moskowitz A, and Connolly J (2001): The contribution of early traumatic events to schizophrenia in some patients: a traumagenic neurodevelopmental model. Psychiatry 64:319-45.

22. Van Winkel R, Esquivel G, Kenis G, Wichers M, Collip D, Peerbooms O, et al. (2010): REVIEW: Genome-wide findings in schizophrenia and the role of gene-environment interplay. CNS Neurosci Ther 16:e185-92.

23. Abdolmaleky HM, Cheng KH, Faraone SV, Wilcox M, Glatt SJ, Gao F, et al. (2006): Hypomethylation of MB-COMT promoter is a major risk factor for schizophrenia and bipolar disorder. Hum Mol Genet 15:3132-45. 
24. Roffman JL, Gollub RL, Calhoun VD, Wassink TH, Weiss AP, Ho BC, et al. (2008): MTHFR 677C --> T genotype disrupts prefrontal function in schizophrenia through an interaction with COMT 158Val --> Met. Proc Natl Acad Sci U S A 105:17573-8.

25. Mill J, Tang T, Kaminsky Z, Khare T, Yazdanpanah S, Bouchard L, et al. (2008): Epigenomic profiling reveals DNA-methylation changes associated with major psychosis. The American Journal of Human Genetics 82:696-711.

26. Van Os J, Rutten BPF, and Poulton R (2008): Gene-environment interactions in schizophrenia: review of epidemiological findings and future directions. Schizophrenia Bulletin 34:1066.

27. van Os J, Linscott RJ, Myin-Germeys I, Delespaul P, and Krabbendam L (2009): A systematic review and meta-analysis of the psychosis continuum: evidence for a psychosis proneness-persistence-impairment model of psychotic disorder. Psychol Med 39:179-95.

28. Johns LC and van Os J (2001): The continuity of psychotic experiences in the general population. Clin Psychol Rev 21:1125-41.

29. Binder EB, Bradley RG, Liu W, Epstein MP, Deveau TC, Mercer KB, et al. (2008): Association of FKBP5 polymorphisms and childhood abuse with risk of posttraumatic stress disorder symptoms in adults. Jama 299:1291-305.

30. Read J, van Os J, Morrison AP, and Ross CA (2005): Childhood trauma, psychosis and schizophrenia: a literature review with theoretical and clinical implications. Acta Psychiatrica Scandinavica 112:330-50.

31. De Bellis MD, Chrousos GP, Dorn LD, Burke L, Helmers K, Kling MA, et al. (1994): Hypothalamic-pituitary-adrenal axis dysregulation in sexually abused girls. Journal of Clinical Endocrinology \& Metabolism 78:249-55.

32. Tarullo AR and Gunnar MR (2006): Child maltreatment and the developing HPA axis. Hormones and Behavior 50:632-9.

33. Morrison AP, French P, Walford L, Lewis SW, Kilcommons A, Green J, et al. (2004): Cognitive therapy for the prevention of psychosis in people at ultra-high risk: randomised controlled trial. The British Journal of Psychiatry 185:291.

34. Bach P and Hayes SC (2002): The use of acceptance and commitment therapy to prevent the rehospitalization of psychotic patients: a randomized controlled trial. J Consult Clin Psychol 70:1129-39.

35. Myin-Germeys I and van Os J (2007): Stress-reactivity in psychosis: evidence for an affective pathway to psychosis. Clin Psychol Rev 27:409-24.

36. Lataster T, Valmaggia, L., Lardinois, M., Mengelers, R., van Os, J. \& Myin-Germeys, I. (submitted): Stress-reactivity and cognitive impairments: separate mechanisms underlying different symptoms in psychosis.

37. Mata I, Arranz MJ, Staddon S, Lopez-Ilundain JM, Tabares-Seisdedos R, and Murray RM (2006): The high-activity Val allele of the catechol-O-methyltransferase gene predicts greater cognitive deterioration in patients with psychosis. Psychiatric Genetics $16: 213$.

38. Ohnishi T, Hashimoto R, Mori T, Nemoto K, Moriguchi Y, lida H, et al. (2006): The association between the Val158Met polymorphism of the catechol-O-methyl transferase gene and morphological abnormalities of the brain in chronic schizophrenia. Brain 129:399.

39. Lataster T, Collip D, Lardinois M, van Os J, and Myin-Germeys I (2010): Evidence for a familial correlation between increased reactivity to stress and positive psychotic symptoms. Acta Psychiatr Scand 122:395-404.

40. Myin-Germeys I and van Os J (2007): Stress-reactivity in psychosis: evidence for an affective pathway to psychosis. Clin Psychol Rev 27:409-24.

41. Petricoin E, Wulfkuhle J, Espina V, and Liotta LA (2004): Clinical proteomics: revolutionizing disease detection and patient tailoring therapy. Journal of proteome research 3:209-217.

42. Crijns APG, Duiker EW, and De J (2006): Molecular prognostic markers in ovarian cancer: toward patient tailored therapy. International Journal of Gynecological Cancer 16:152-165.

43. Myin-Germeys I, Birchwood M, and Kwapil T (2011): From environment to therapy in psychosis: a real-world momentary assessment approach. Schizophr Bull 37:244-7.

44. Thewissen V, Bentall RP, Lecomte T, van Os J, and Myin-Germeys I (2008): Fluctuations in self-esteem and paranoia in the context of daily life. J Abnorm Psychol 117:143-53.

45. Thewissen V, Bentall RP, Oorschot M, Campo JA, van Lierop T, van Os J, et al. (2010): Emotions, self-esteem, and paranoid episodes: An experience sampling study. The British Journal of Clinical Psychology DOI: 10.1348/014466510X508677. 


\section{Summary \\ Samenvatting}




\section{Summary}

Increased sensitivity to stress has been proposed as a vulnerability marker for psychosis. Moreover, increased stress sensitivity is particularly associated with positive psychotic symptoms, at clinical as well as subclinical levels. This thesis "Interactions in Psychosis - Linking environment, brain and genes", tries to further explore underlying mechanisms of clinical and subclinical positive psychotic symptoms as well as underpinnings of the stress sensitivity endophenotype of psychosis.

Chapter 1 provides an introduction to psychotic disorders. The phenomenology of psychotic disorders and its heterogeneity is described. It is argued that an extended psychosis phenotype exists in nature that often consists of transient psychotic experiences and shows etiological and familial continuity with clinical psychosis. Moreover, evidence is reviewed putting increased stress sensitivity forward as an underlying vulnerability marker for a stress reactive subtype of psychotic disorders with high levels of positive psychotic symptoms. Moreover, we present the importance of social factors, including environmental exposures as well as interpersonal functioning, in the development of subclinical and clinical positive psychotic symptoms. In addition, potential underlying biological markers of increased stress reactivity, including HPA axis functioning, hippocampal volume and COMT genotype, are promoted. The chapter finishes with the aims and outline of the thesis.

The present thesis consists of two parts. In the first part (chapter 2, 3 and 4) we examined the role and mechanisms of social factors in the development of clinical and subclinical positive psychotic symptoms.

In chapter 2, epidemiological research is reviewed that has shown that stressful environmental factors can play an etiological role in the development of psychosis. However, the mechanism underlying the link between stress and psychosis is still not fully understood. It is argued that the interaction between stressful environmental factors and (epi)genetic factors can bring about different psychological and biological alterations. Both types of alterations can be referred to as a process called 'sensitization'. The underlying mechanism of sensitization can be interpreted on the one hand as cognitive misinterpretations (psychological sensitization) and on the other hand as altered dopaminergic neurotransmission (biological sensitization). Both of these deviations can facilitate the onset and persistence of psychotic symptoms. The chapter finishes with arguing that, at the psychometric level, sensitization can be quantified as (i) stress-induced persistence of the normally transient expression of subclinical psychotic experiences during adolescence and early adulthood and as (ii) the increased risk of transition from gradually more persistent subclinical psychotic experiences to a clinical psychotic disorder. 
Chapter 3 presents a longitudinal study, in which the bi-directional association between interpersonal functioning and several subclinical symptom dimensions was examined. Cross-lagged path modelling was used to analyse bi-directional, longitudinal associations between four dimensions of subclinical psychotic experiences (Persecutory ideation, Bizarre experiences, Perceptual abnormalities, Magical thinking) and interpersonal functioning in an adolescent general population sample ( $\mathrm{N}=813)$, followed three times over three years. The results suggest that, while all symptom dimensions showed some association with interpersonal functioning over time, only Bizarre experiences and Persecutory ideation were consistently longitudinally associated with interpersonal functioning. A lower level interpersonal functioning predicted higher levels of Bizarre experiences and Persecutory ideation at later measurement points (both T1 to T2 and T2 to T3). Hence, within the domain of subthreshold experiences of psychosis during adolescence, a decrease in interpersonal functioning may form the starting point of a deteriorating development predicting persecutory ideas and bizarre experiences in particular.

Chapter 4 focused on the microenvironment of everyday life to examine associations between social context and paranoid experiences. The hypothesis that psychometric paranoia risk would moderate the association between contextual variables and momentary increases in the intensity of paranoid thinking was examined in a sample $(n=154)$ consisting of 30 currently paranoid patients, 34 currently non-paranoid patients, 15 remitted psychotic patients, 38 high-schizotypy participants, and 37 control subjects of which three groups with different degrees of paranoia were defined. Momentary social context, perceived social threat and paranoia in daily life were assessed using ESM. The results demonstrated differences in the effect of social company on momentary levels of paranoia and perceived social threat across the range of trait paranoia. The low and medium paranoia groups reported higher levels of perceived social threat when they were with less-familiar compared to familiar individuals. The medium paranoia group reported more paranoia in less-familiar company. The high paranoia group, however, reported no difference in the perception of social threat or momentary paranoia between familiar and unfamiliar contacts. These results suggest that paranoid thinking is context dependent in individuals with medium or at-risk levels of trait paranoia. Perceived social threat seems to be context dependent in the low paranoia group. At high levels of trait paranoia, however, momentary paranoia and momentary perceived social threat become autonomous and independent of social reality.

The second part of this thesis (chapter 5, 6 and 7) concentrates on biological and genetic markers of positive psychotic symptoms and increased stress sensitivity.

The aim of the study described in Chapter 5 was to investigate whether HPA axis functioning is altered in individuals at above average genetic risk for psychotic disorder, examining diurnal cortisol profiles, cortisol reactivity to daily stressors and the association between HPA axis activity and subclinical 
psychotic experiences. Siblings of patients with a psychotic disorder $(n=60)$ and a healthy comparison group ( $n=63$ ) participated in an ESM study in which we assessed stress, psychotic experiences, negative affect and salivary cortisol repeatedly in the flow of daily life. Results revealed higher diurnal cortisol levels and heightened cortisol reactivity to negative daily events in siblings compared with controls. Diurnal cortisol slope did not differ between the two groups, but momentary increases in psychotic experiences and negative affect were associated with increased cortisol in the sibling group. Thus, altered HPA axis activity seems to be present in individuals at above average genetic risk for psychotic disorder. Results also suggest a dynamic association between cortisol secretion and the intensity of psychotic-like experiences and negative emotions in daily life, although the direction of this association remains to be elucidated.

The study presented in Chapter 6, investigated the association between hippocampal volume and daily life stress reactivity, indexed by negative emotions as well as by cortisol reactions to daily hassles, and whether genetic risk (the contrast between controls, patients and siblings of patients) moderated this relationship. Hippocampal volume was measured in 20 patients with schizophrenia, 38 healthy siblings at higher than average genetic risk for schizophrenia and 33 controls. ESM in combination with salivary sampling was used to assess emotional stress reactivity (the effect of momentary stress on momentary negative affect) and, for the control and sibling groups, cortisol stress reactivity. Results revealed that the association between (emotional and cortisol ) stress sensitivity and hippocampal volume was different between the groups. Increased emotional stress reactivity was associated with smaller left hippocampal volume in patients and larger total hippocampal volume in controls. In line with the results in patients, siblings with small hippocampal volume demonstrated increased emotional and cortisol stress reactivity compared to those with large hippocampal volume. Thus, the findings suggest that hippocampal volume may index risk and possibly disease-related mechanisms underlying daily life emotional and cortisol stress reactivity in psychotic disorder.

The goal of the study in Chapter 7 was to investigate whether psychosis risk (patient vs. control) moderated the association between (emotional and psychotic) stress reactivity and the COMT Val158Met polymorphism. In case of a significant interaction, the aim was to examine how the COMT Val158Met polymorphism moderates affective and psychotic reactions to momentary stress within the two groups. In patients with a non-affective psychotic disorder $(n=89)$ and control participants $(n=127)$ ESM was administered to examine stress, negative affect and momentary psychotic symptoms in the flow of daily life. Results demonstrated group (patient or control) differences in the effect of COMT genotype on stress reactivity. In patients, COMT genotype was associated with emotional as well as psychotic reactivity to momentary stress. Met/Met genotype patients showed significantly increased psychotic and emotional reactivity to stress in comparison to the Val/Met and Val/Val genotypes. In contrast, healthy controls did not display large or significant COMT Val158Met X stress interactions. 
Thus, important differences might exist in the effect of COMT Val158Met on stress reactivity, which may depend on background risk for psychotic disorder.

In Chapter 8, the results of this thesis are discussed and directions for future research are provided. The results of the different studies are integrated and differences in mechanisms along the psychosis continuum are discussed. Finally, some clinical implications of the findings in this thesis are provided. 


\section{Interactions in psychosis - linking environment, brain and genes}

\section{Samenvatting}

Uit epidemiologisch onderzoek is gebleken dat stress een rol spelt bij het ontstaan van psychotische stoornissen. Stressvolle gebeurtenissen, zoals trauma tijdens de kindertijd, kunnen leiden tot verhoogde gevoeligheid voor stress op latere momenten in het leven. Verschillende studies hebben aangetoond dat een verhoogde stressgevoeligheid vooral voorkomt bij die subgroep van patiënten, die vooral veel last heeft van positieve psychotische symptomen.

We weten echter nog onvoldoende van het mechanisme achter het verband tussen stress en psychose. In mijn proefschrift met de titel "Interactions in Psychosis - Linking environment, brain and genes" hebben we daarom biologische en genetische markers van stressgevoeligheid en mechanismen die ten grondslag liggen aan psychotische ervaringen onderzocht.

Hoofdstuk 1 beschrijft de fenomenologie en heterogeniteit van psychotische stoornissen en introduceert het continuüm model van psychotische ervaringen. Dit model veronderstelt dat het psychose fenotype zowel subklinische psychoseachtige ervaringen die voorkomen in de algemene bevolking als klinische psychoses omvat. Subklinische psychotische ervaringen zijn vaak van korte duur en liggen op één continuüm met klinische psychose, qua etiologie en erfelijke factoren. Verder wordt een verhoogde stress gevoeligheid geïntroduceerd als een marker van psychotische stoornissen. We presenteren het belang van sociale factoren, omgevingsfactoren en interpersoonlijk functioneren bij het ontstaan van subklinische en klinische positieve psychotische symptomen. Ook beschrijven we welke mechanismen aan een verhoogde stress reactiviteit ten grondslag kunnen liggen, zoals het functioneren van de HPAas, het volume van de hippocampus en het COMT genotype. Het hoofdstuk sluit met de doelen en de opzet van dit proefschrift.

In het eerste deel van dit proefschrift (hoofdstuk 2, 3 en 4) hebben we de rol van sociale factoren bij het ontstaan van klinische en subklinische positieve psychotische symptomen onderzocht.

In hoofdstuk 2 wordt beschreven hoe epidemiologisch onderzoek heeft aangetoond dat stressvolle omgevingsfactoren een etiologische rol kunnen spelen bij de ontwikkeling van psychose. Het mechanisme dat ten grondslag ligt aan de associatie tussen stress en psychose wordt echter nog onvoldoende begrepen. In dit hoofdstuk wordt beargumenteerd dat stressvolle omgevingsfactoren, in interactie met (epi)genetische factoren, psychologische en biologische veranderingen teweeg kunnen brengen die samen zijn te vatten onder de noemer 'sensitisatie'. Er worden twee aspecten van sensitisatie onderscheiden: psychologische en biologische sensitisatie. Psychologische sensitisatie kan begrepen worden als cognitieve vertekeningen en biologische sensitisatie kan worden opgevat als 
veranderde dopaminerge neurotransmissie. Sensitisatie kan met behulp van epidemiologisch onderzoek op psychometrisch niveau worden gekwantificeerd als stressgeïnduceerde persistentie (als indicator van blijvende sensitisatie) van de normaliter voorbijgaande expressie van subklinische psychotische ervaringen tijdens de adolescentie en vroege volwassenheid.

Hoofdstuk 3 presenteert een longitudinale studie, met drie meetmomenten over een periode van drie jaar, waarin de associatie tussen interpersoonlijk functioneren en subklinische psychotische ervaringen is onderzocht. In een steekproef van jongeren uit de algemene bevolking ( $N=875)$, hebben wij de relatie tussen met vier subdimensies van subklinische psychotische ervaringen (achterdochtige ideeën, bizarre ervaringen, perceptuele afwijkingen, magisch denken) en interpersoonlijk functioneren (met betrekking tot familie en leeftijdsgenoten) gemodelleerd. De resultaten tonen aan dat verminderd interpersoonlijk functioneren vooral het ontstaan van bizarre ervaringen en achterdochtige ideeën voorspelt. De kwaliteit van de relatie met leeftijdsgenoten was vooral geassocieerd met bizarre ervaringen, terwijl de kwaliteit van de relatie met de familie de sterkste associatie met achterdochtige ideeën liet zien. Een verslechtering in interpersoonlijk functioneren tijdens de adolescentie zou dus ten grondslag kunnen liggen aan het ontstaan van psychotische ervaringen.

In Hoofdstuk 4 onderzochten wij de associatie tussen de sociale context en paranoïde ideeën, in de micro-omgeving van het dagelijks leven. We onderzochten hoe gevoeligheid voor psychotische ervaringen de sterkte van deze associatie beïnvloedt. Een groep van 154 mensen (bestaande uit mensen met en zonder een psychotische stoornis) werd ingedeeld in drie groepen met verschillende mate van gevoeligheid. Met behulp van de Experience Sampling Method (ESM) onderzochten we de invloed van sociale context (samenzijn met bekenden versus met onbekenden), ervaren sociale dreiging en paranoïde ideeën in het dagelijkse leven. De resultaten lieten zien dat het effect van sociaal gezelschap op paranoïde ideeën verschilt per groep. De groepen met een lage of gemiddelde gevoeligheid voor paranoia ervoeren meer sociale dreiging en paranoïde ideeën wanneer ze met onbekenden samen waren dan wanneer ze met bekenden samen waren. De groep met de grootste gevoeligheid voor paranoia rapporteerde echter geen verschil in het niveau van sociale dreiging en paranoïde ideeën tussen het samenzijn met bekenden en onbekenden. Paranoïde ervaringen blijken dus context-afhankelijk te zijn in mensen met een laag of gemiddeld risico op paranoia. Bij mensen met een hoog risico op paranoia blijkt het ervaren van paranoïde ideeën en het gevoel van sociale dreiging onafhankelijk te zijn van de sociale context.

In het tweede deel van dit proefschrift (hoofdstuk 5, 6 en 7) wordt gezocht naar biologische en genetische markers voor positieve psychotische symptomen en een verhoogde stress sensitiviteit. 
Hoofdstuk 5 rapporteert een studie die veranderingen in het functioneren van de HPA-as in mensen met een verhoogd genetisch risico op psychose onderzoekt. Hiervoor hebben we de cortisol reacties op dagelijkse stressoren en de associatie tussen HPA-as activiteit en subklinische psychotische ervaringen onderzocht. Aan deze ESM studie namen 60 broers en zussen van patiënten met een psychotische stoornis en 63 gezonde controle personen deel. Met behulp van ESM hebben wij stress, psychotische ervaringen, negatieve emoties en cortisol (in speeksel) in het dagelijks leven gemeten, op meerdere momenten per dag. We vonden verhoogde cortisol waarden en een verhoogde cortisol reactie op dagelijks stress in mensen met een genetische kwetsbaarheid voor psychose. Deze verhoogde cortisol reactie was ook geassocieerd met het hebben/rapporteren van subklinische psychotische ervaringen. Veranderde HPA-as activiteit lijkt dus aanwezig te zijn in mensen met een verhoogde genetische kwetsbaarheid voor psychose.

De resultaten doen ook vermoeden, dat er een associatie bestaat tussen de afgifte van cortisol en de intensiteit van subklinische psychotische ervaringen en negatieve emoties in het dagelijks leven. De richting van deze associatie moet echter nog verder onderzocht worden.

In hoofdstuk 6 onderzochten we de associatie tussen het volume van de hippocampus en dagelijkse stress reactiviteit (emotionele en cortisol reacties op dagelijkse stress). Ook onderzochten we of deze associatie wordt beïnvloed door het genetisch risico op psychose. Het hippocampus volume werd gemeten in 20 patiënten met een psychotische stoornis, 38 gezonde broers en zussen van patiënten met een psychose (zij hebben dus een hoger dan gemiddeld genetisch risico voor psychotische stoornissen) en 33 controle personen. ESM werd gebruikt om emotionele stress reactiviteit te meten. In zowel de controle als de broers en zussen groep werd ook speeksel verzameld om cortisol stress reactiviteit te meten. De resultaten lieten zien dat de associatie tussen (emotionele en cortisol) stress sensitiviteit en hippocampus volume verschillend was tussen de groepen. Een verhoogd emotionele stress reactiviteit was geassocieerd met kleiner hippocampus volume in patiënten en een groter hippocampus volume in controles. Net zoals patiënten, lieten broers en zussen met een kleiner hippocampus volume de grootste emotionele en cortisol stress reactiviteit zien. Deze resultaten suggereren dat hippocampus volume geassocieerd is met risico gerelateerde patronen van dagelijkse emotionele en cortisol stress reactiviteit in mensen met een verhoogd risico voor psychotische stoornissen.

Hoofdstuk 7 beschrijft een studie waarin we de gen-omgevingsinteractie tussen het Cathechol-OMethyltransferase Val158 Met polymorphisme (COMT) en stress onderzochten. Eerdere studies naar deze gen-omgevingsinteractie hadden inconsistente bevindingen. We hebben dit onderzocht met behulp van ESM in 89 patiënten met een psychotische stoornis en 127 gezonde proefpersonen. In de analyses onderzochten we de invloed van het COMT Val ${ }^{158} \mathrm{Met}$ genotype op stress-geïnduceerde psychotische fenomenen (psychotische stress-reactiviteit). We vonden dat de interactie tussen stress 
en COMT verschillend was in de patiënten- en controlegroep. In de patiënten groep was er sprake van een significante interactie tussen COMT Val ${ }^{158}$ Met genotype en stress, maar dit was niet het geval in de controlegroep. Patiënten met het Met/Met genotype lieten een sterkere psychotische reactie zien na stressvolle gebeurtenissen dan patiënten met het Val/Met of Val/Val genotype. Het effect van dagelijkse stress op psychose is dus afhankelijk van COMT Val ${ }^{158}$ Met genotype en diagnostische groep. Met behulp van deze resultaten, werden eerdere studies die een verhoogde stressgevoeligheid in patiënten met een Met/Met genotype aantoonden, gerepliceerd. Opvallend is de sterkere psychotische reactie op stress in de patiënten met het Met/Met genotype, die mogelijk verklaard kan worden met behulp van de epigenetica. Kortom, er zijn belangrijke verschillen in het effect van COMT Val158Met op stress reactiviteit, die afhankelijk zijn van het genetisch risico op psychose.

In Hoofdstuk 8 worden de belangrijkste bevindingen uit dit proefschrift samengevat en worden suggesties voor verder onderzoek gegeven. Resultaten van de verschillende studies worden geïntegreerd en de verschillen tussen mechanismen in de verschillende fases van het psychose continuüm worden besproken. Tot slot worden enkele klinische implicaties van deze bevindingen gegeven. 



\title{
A cknowledgements
}

\author{
Dankwoord \\ Dankwort
}


In elke fase van mijn leven hebben veel mensen mij gesteund en mede dank zijn hun is dit proefschrift tot stand gekomen. ledereen die een bijdrage heeft geleverd wil ik hiervoor heel hartelijk danken.

Allereerst wil ik graag mijn promotoren, Prof. Dr. Inez Myin-Germeys en Prof. Dr. Jim van Os danken. Beste Inez, bedankt voor de mogelijkheid om als AIO aan jouw VIDI project te werken. Zonder jou was dit proefschrift niet tot stand gekomen. Van harte dank, voor alles dat je me leerde en voor de vele mogelijkheden die ik heb gekregen. Bedankt ook voor jouw gedrevenheid en enthousiasme. Je wist altijd wat je moest zeggen, om me te motiveren als het een keertje niet helemaal naar wens liep. Het geloof en vertrouwen dat je steeds in mij hebt gehad, heeft me vaak geholpen om mijn geloof in mezelf terug te vinden. Jim, het is leuk om te zien hoe je met jouw oneindige enthousiasme zoveel mensen voor de wetenschap inspireert. Dank ervoor! Dank ook voor jouw superhandige en snelle feedback voor artikelen - ze zijn er zo veel beter van geworden. Ook wil ik je bedanken, voor jouw missie om SP zo'n fijne plek te maken. Ik voel me vereerd om bij jou in de onderzoeksgroep te hebben mogen werken.

I would also like to thank the members of the advisory board. Ook wil ik alle leden van de leescommissie en corona bedanken.

Nancy, bedankt voor de superfijne samenwerking bij de cortisol studies. Ik heb van je veel geleerd en ben er heel erg dankbaar voor. I admire your spirit of research!

Ruud, dank voor jouw geduld wat betreft al mijn, soms waarschijnlijk naïeve, vragen over genetica. Bedankt voor de fijne samenwerking!

Johan, my teammate! Dank je wel voor een heel leuke tijd samen in een kamer! Volgens mij hebben we allebei mazzel gehad, dat we even chaotisch en "minder" netjes zijn dan de gemiddelde AIO (anders was een van ons waarschijnlijk al lang van kamer gewisseld). Dank ook voor het leuke brainstormen, de vele gesprekken en het lachen. Ik vind dat we elkaar ook wisten aan te vullen en ik ben er trots op dat we tijdens het STRIP project ondanks moeilijkheden toch eigenlijk alle problemen hebben opgelost en het project nog aan een succesvol einde hebben gebracht. Dank je voor alles!

Maggy, ik vond het superleuk om met jou samen te werken (... minstens zo leuk als een bed te delen ;-). Ik vind dat we een geweldig team zijn... mijn chaotische en jouw gestructureerde en nette manier maken een mooi geheel. Ik hoop en verheug me erop om in de toekomst nog aan veel projecten met je samen te werken. Dank ook voor de leuke gesprekken, jouw onophoudelijke hulp en dat je er altijd voor mij bent geweest.

Becca und Anne, schön dass ihr nicht nur während dem Master sondern auch noch während der AIO Periode an meiner Seite ward. Danke für die „geselligen“ etentjes, Kongressbesuche, die gemeinsamen Fahrten zur Arbeit und und und... Nicole, schön Dich nebenan im Zimmer zu wissen, danke für die schöne Zeit.

Wendy, je bent een engel! Dank voor al je hulp bij alles :-). 
Truda, van harte dank voor jouw hulp bij het opstarten van de STRIP studie. Dank ook aan het datamanagement team en de data invoer. Trees, Jolanda, Ine en Leni, bedankt voor de administratieve ondersteuning en dat er altijd iets lekkers voor de medewerkers was. Jolanda, van harte bedankt vooral ook voor jouw steun bij het "administratieve" afronden van mijn proefschrift! Ron, bedankt voor al het programmeerwerk en het in stand houden van mijn computer. Tineke, dank je dat ik altijd met vragen bij jou terecht kon en voor je hulp bij klussen zoals bv een abstract van 450 woorden naar 250 in te korten. Gunther, dank voor de fijne samenwerking en het snelle afhandelen van 'ons' DNA. Marieke dank je dat ik altijd bij jou mocht aankloppen, of het nu over statistische vragen of toekomstgerelateerd advies ging.

Claudia, Feikje, Karin, Frieda, Koen, leuk dat jullie er allemaal waren - het heeft een verschil gemaakt!

Frenk, dank voor jouw enthousiaste begeleiding bij mijn afstudeertheses - ik heb daardoor zin in de wetenschap gekregen!

Alle AIOs wil ik bedanken voor de fijne samenwerking en al de gezelligheid in de laatste jaren.

Rufa, superleuk dat je ook nog deel hebt uitgemaakt van het STRIP team- bedankt voor je inzet en hulp bij de dataverzameling.

Carmen - superbedankt voor je hulp bij het klaarmaken van de cortisol staaltjes!

Dank aan iedereen die ook nog een bijdrage aan de STRIP studie heeft geleverd, vooral David en Bernice. Bernice, leuk waren de reizen naar Leuven, de voetbal gesprekken, etentjes \& onze STRIP- salsa.

Odette, ik vond het heel gezellig om samen met jedoor de dataset te worstelen voor onze GxE analyses :-) was veel leuker als team dan het alleen was geweest!

Petra, Machteld en Ed, ik heb genoten van onze samenwerking en ben er helemaal blij mee! Petra, dank ook voor jouw Indesign crash cursus.

Hanneke! Dank voor de vele koffie en thees. Dank voor de superfijne samenwerking aan ons artikel, de gezellige planning van ons symposium... vooral ook bedankt voor jouw superlieve ontvangst en zorg in Australië!

Alison, Ash, Barnaby - thanks for the opportunity to work with an Australian dataset \& thanks for the time in Melbourne!

Tevens wil ik alle andere co-auteurs bedanken voor de prettige samenwerking en voor de gelegenheid die ik in de afgelopen jaren heb gekregen om met andere datasets te mogen werken. Viviane en Marielle, superleuk dat ik met het ZAPP en NARSAD dataset mocht werken. Dank voor de fijne samenwerking!

Ook wil ik alle andere collegas van SP bedanken. Dank voor het gezellige zingen en voor de leuke sfeer op SP! 
Vooral wil ik ook alle proefpersonen bedanken die hebben meegewerkt bij de STRIP studie en alle andere studies. Zonder jullie inzet zou al dit mooie onderzoek niet tot stand zijn gekomen!

Liebe Freunde und Familie, von Herzen Danke ich euch. Jeder hat auf seine Weise einen Teil zu diesem Büchlein beigetragen.

Claudia, Frauke, Nina, Nick, ...und all die anderen: danke für die Zeit in Maastricht, das Studium... es tat gut euch an meiner Seite zu wissen, ihr habt mich immer wieder ermutigt meinen Weg zu finden.

Anne, Melanie, Kathrin, Olli, Christina, Stefan, Marcel, Tilman, Thomas, Phillip und Christian, schoen, dass Ihr mich auf meinem Weg begleitet habt!

Liebe Inga, danke für die schönen Gespräche und den Austausch zwischen Forschung und Klinik, zwischen Holland und Deutschland, zwischen Dir und mir.

Omas und Opas, danke dass ihr immer Interesse an mir und auch meiner Arbeit habt, und auch wenn es für euch manchmal sehr abstrakt sein muss, ihr euch immer alles angehört und mir immer mit einem guten Rat zur Seite gestanden habt.

Liebes Brüderchen, schön dass es Dich gibt. Danke, dass du immer wieder versucht hast mich daran zu erinnern, dass die Wochentage zum Arbeiten und die Wochenenden zum Ausruhen gedacht sind :-)

Liebe Mama, lieber Papa, es gibt keine Worte für eure Liebe und Euer Vertrauen in mich und für alle Chancen und Möglichkeiten die Ihr mir gegeben habt. Von Herzen Danke für alles.

Mein liebster Sven, danke für alles (auch für die viele Hilfe bei dem Layout dieses Büchleins). Du hilfst mir meinen Blick nicht in Hypothesen und Datensets zu verlieren; danke dass es Dich gibt! 


\section{Curriculum Vitae}

Dina Collip was born on August 27, 1981 in Aachen, Germany. In 2001 she graduated from secondary school (Abitur) at the Staedtisches Gymnasium Eschweiler, Germany. In 2002, she started studying Psychology at Maastricht University where she received a Bachelor of Arts in Biological Psychology (with merit) in 2005. As part of her Bachelors, she spent 5 months studying at the University of California, Santa Barbara. Back in Maastricht, she started the Research Master in Neuroscience, Neuropsychology and Abnormal Psychology. During her Masters she spent 5 months at the University of British Columbia in Vancouver, Canada working on the research for her Master thesis entitled "The Cortisol/DHEA molar ratio and BDNF in euthymic bipolar patients". Thereafter, she spent 3 months in Heidelberg, Germany for her clinical internship and the research project for her minor research thesis entitled "Psychometric Properties of the Almost Perfect Scale-Revised (APS-R): Reliability and Construct Validity of the original 3-Factor Structure in a German-Speaking Depressed Sample". In 2007 she received her Master's degree (cum laude).

From November 2007 until October 2011 she was a PhD candidate at the School for Mental Health and Neuroscience (division Mental Health) within the Department of Psychiatry and Neuropsychology at Maastricht University. During her PhD, she worked for three months as a guest member of staff at Orygen Youth Health Research Center at Melbourne University, Australia. 


\section{List of publications}

\section{International journals}

Collip D, Myin-Germeys I, Van Os J (2008) Does the concept of „sensitization“ provide a plausible mechanism for the putative link between the environment and schizophrenia? Schizophr Bull, 34(2): 220-225.

Myin-Germeys I, Collip D, Oorschot M, Lataster J, Delespaul P, van Os J (2009) Experience sampling research in psychopathology: opening the black box of daily life. Psychol Med, 39(9): 1533-1547.

Collip D, Oorschot M, Thewissen V, Van Os J, Bentall R, Myin-Germeys I (2010a) Social world interactions: how company connects to paranoia. Psychol Med: 1-11.

Collip D, Van Winkel R, Peerbooms O, Lataster T, Thewissen V, Lardinois M, Drukker M, Rutten BP, Van Os J, Myin-Germeys I (2010b) COMT Val158Met-Stress Interaction in Psychosis: Role of Background Psychosis Risk. CNS Neurosci Ther.

Lataster T, Collip D, Lardinois M, van Os J, Myin-Germeys I (2010) Evidence for a familial correlation between increased reactivity to stress and positive psychotic symptoms. Acta Psychiatrica Scandinavica, 122(5): 395-404.

Van Winkel R, Esquivel G, Kenis G, Wichers M, Collip D, Peerbooms O, Rutten B, Myin-Germeys I, Van Os $J$ (2010) REVIEW: Genome-wide findings in schizophrenia and the role of gene-environment interplay. CNS Neurosci Ther, 16(5): e185-192.

Collip D, Nicolson NA, Lardinois M, Lataster T, van Os J, Myin-Germeys I (2011) Daily cortisol, stress reactivity and psychotic experiences in individuals at above average genetic risk for psychosis. Psych Med, 1(-1): 1-11.

Lataster J, Collip D, Ceccarini J, Haas D, Booij L, van Os J, Pruessner J, van Laere K, Myin-Germeys I (accepted) Psychosocial stress is associated with in vivo dopamine release in human ventromedial prefrontal cortex: a positron emission tomography study using [18F]fallypride. Neurolmage.

Collip D, Wigman JTW, Lin A, Nelson B, Oorschot M, Vollebergh WAM, Ryan J, Baksheev G, Wichers M, van Os J, Myin-Germeys I, Yung AR (accepted) Dynamic association between Interpersonal Functioning \& Positive Symptom Dimensions of Psychosis over Time: a Longitudinal Study of Healthy Adolescents. Schizophr Bull. 


\section{National journals}

Collip D, Myin-Germeys I, Van Winkel R, van Os J (2009) [Stress and psychosis: is sensitisation the underlying mechanism?]. Tijdschr Psychiatr, 51(8): 559-567.

\section{Submitted papers}

Collip D, Habets P, Marcelis M, Gronenschild E, Lataster T, Lardinois M, Nicolson NA, van Os J, MyinGermeys I, for G.R.O.U.P. Hippocampal Volume as Marker of Daily Life Emotional and Cortisol Stress Sensitivity in Psychosis. Submitted.

Collip D, Habets P, Myin-Germeys I, Gronenschild E, van Bronswijk S, Hofman P, Lataster T, Lardinois M, Nicolson NA, van Os J, Marcelis M, for G.R.O.U.P. Pituitary volume, stress-reactivity and genetic risk for psychotic disorder. Revision pending Psych Med.

Peerbooms O, Collip D, Rutten B, Lardinois M, Lataster T, Thewissen V, Mafirad S, Drukker M, Kenis G, van Os J, Myin-Germeys I, van Winkel R. Interactive effects of COMT and MTHFR moderate stress sensitivity in psychosis. Submitted.

Lataster J, Collip D, Ceccarini J, Hernaus D, Haas D, Booij L, van Os J, Pruessner J, van Laere K, MyinGermeys I. Aberrant dopamine stress processing in ventromedial prefrontal cortex of individuals at genetic risk for psychosis. Submitted.

Hernaus D, Lataster J, Collip D, Ceccarini J, Haas D, Booij L, van Os J, Pruessner J, van Laere K, MyinGermeys I. Psychotic reactivity to daily life stress is associated with stress-induced in vivo prefrontal hypodopaminergia: evidence from Experience Sampling and [18F]fallypride PET data. Submitted. 


\section{Abstracts and presentations}

\section{National}

Collip D, Habets P, Marcelis M, Gronenschild E, Lataster T, Lardinois M, Van Os J, Myin-Germeys I Hippocampal volume predicting emotional and biological stress-reactivity in psychosis. 2011 Research Day School for Mental Health and Neuroscience. Poster presentation.

Collip D, Wichers M, Delespaul P, Myin-Germeys I. Experience Sampling Method -Background. 2011 Voorjaarscongress (National Psychiatry Conference), Nederlands Vereniging voor Psychiatrie, Amsterdam, The Netherlands. Oral presentation.

Collip D, Van Winkel R, Peerbooms O, Lataster T, Thewissen V, Lardinois M, Drukker M, Rutten BP, Van Os J, Myin-Germeys I. COMT Val158Met-Stress Interaction in Psychosis: Role of Background Psychosis Risk. 2010 Voorjaarscongress (National Psychiatry Conference), Nederlands Vereniging voor Psychiatrie, Maastricht, The Netherlands. Oral presentation.

Collip D, Van Winkel R, Peerbooms O, Lataster T, Thewissen V, Lardinois M, Drukker M, Rutten BP, Van Os J, MyinGermeys I. COMT Val158Met-Stress Interaction in Psychosis: Role of Background Psychosis Risk. 2010 Research Day School for Mental Health and Neuroscience. Poster presentation.

Collip D, Oorschot M, Thewissen V, Van Os J, Bentall R, Myin-Germeys I. Social world interactions: how company connects to paranoia. 2009 Research Day School for Mental Health and Neuroscience. Poster presentation.

Collip D, Lataster T, Van Os J, Myin-Germeys I. Is increased reactivity to stress an endophenotype for psychosis? 2008 Research Day School for Mental Health and Neuroscience. Poster presentation.

Collip D., Mclsaac, S., Kuan, A., Young, A.H. (2007). Stress Hormones, BDNF and Cognition in Euthymic Bipolar Patients. 6th Dutch Endo-Neuro-Psycho Meeting, Doorwerth, the Netherlands. Oral presentation.

Habets P, Collip D, Gronenschild E, Van Bronswijk S, Van Os J, Myin-Germeys I, Marcelis M. Stress sensitiviteit en brein veranderingen in psychose: Bewijs voor verstorde functie van de Hypothalamus-Hypofyse-Bijnieras? 2010; Schizofreniecongres Zwolle.

Habets P, Collip D, Myin-Germeys I, Gronenschild E, Lardinois M, Lataster T, Van Bronswijk S, Van Os J, Marcelis M (2011) Pituitary volume, stress-reactivity and genetic risk for psychotic disorder. 2011 Research Day School for Mental Health and Neuroscience 


\section{International}

Collip D, Habets P, Marcelis M, Gronenschild E, Lataster T, Lardinois M, Van Os J, Myin-Germeys I (2011) Hippocampal volume predicting emotional and biological stress-reactivity in psychosis. Schizophr Bull, 37: 161. International Congress on Schizophrenia Research, Colorado, USA. Poster presentation.

Habets P, Collip D, Myin-Germeys I, Gronenschild E, Lardinois M, Lataster T, Van Bronswijk S, Van Os J, Marcelis M (2011) Pituitary volume, stress-reactivity and genetic risk for psychotic disorder. Schizophr Bull, 37: 166. International Congress on Schizophrenia Research, Colorado, USA.

Collip D, Oorschot M, Thewissen V, Van Os J, Bentall R, Myin-Germeys I. Social world interactions: how company connects to paranoia. 2011; Australian Society for Psychiatric Research (ASPR) 2010 Conference in Sydney, Australia. Poster presentation.

Collip D, Van Winkel R, Peerbooms O, Lataster T, Thewissen V, Lardinois M, Drukker M, Rutten BP, Van Os J, Myin-Germeys I (=presenting author). COMT Val158Met-Stress Interaction in Psychosis: Role of Background Psychosis Risk. 2010; International Early Psychosis Association Conference, Amsterdam. Oral presentation.

Collip D, Van Winkel R, Peerbooms O, Lataster T, Thewissen V, Lardinois M, Drukker M, Rutten BP, Van Os J, MyinGermeys I. COMT Val158Met-Stress Interaction in Psychosis: Role of Background Psychosis Risk. Schizophrenia Research 2010. Schizophrenia International Research Society Conference, Florence, Italy. Poster presentation.

Collip D, Van Winkel R, Peerbooms O, Lataster T, Thewissen V, Lardinois M, Drukker M, Rutten BP, Van Os J, MyinGermeys I. COMT Val158Met-Stress Interaction in Psychosis: Role of Background Psychosis Risk. 2009; DGPPN Congress (Psychiatry Conference), Berlin, Germany. Oral presentation.

Collip D, Oorschot M, Delespaul P, Myin-Germeys I. ESM research in psychosis. 1st Biennial Conference of the Society for Ambulatory Assessment, 2009; Greifswald, Germany. Oral presentation.

Collip D, Oorschot M, Thewissen V, Van Os J, Bentall R, Myin-Germeys I. Social world interactions: how company connects to paranoia. 2009; Schizophrenia Bulletin. International Congress of Schizophrenia, San Diego, USA. Poster presentation.

Collip D, Oorschot M, Thewissen V, Van Os J, Bentall R, Myin-Germeys I. Social world interactions: how company connects to paranoia. 2008; DGPPN Congress (Psychiatry Conference), Berlin, Germany. Poster presentation.

Collip D, Lataster T, Van Os J, Myin-Germeys I. Is increased reactivity to stress an endophenotype for psychosis? Schizophrenia Research 2008; 102. Schizophrenia International Research Society Conference, Venice, Italy. Poster presentation. 
